\title{
Disruption of Antiaromaticity in Structurally Related Expanded Porphyrin-Like Macrocycles with Benzene Linkers
}

Thondikkal Sulfikarali ${ }^{\mathrm{a}}$, Govind Behera ${ }^{\mathrm{a}}$, Jayaprakash Ajay ${ }^{\mathrm{a}}$, Shigeki Mori ${ }^{\mathrm{b}}$, Akhil Chakravarthy Kakarlamudi ${ }^{a}$, Sivaranjana Reddy Vennapusa ${ }^{\mathrm{a}}$ and Sabapathi Gokulnath ${ }^{\mathrm{a} *}$

andian Institute of Science Education and Research Thiruvananthapuram, Kerala-695 016, India. E-mail: gokul@iisertvm.ac.in.

${ }^{b}$ Division of Material Science, Advanced Research Support Center, Ehime University, 2-5 Bunkyo-cho, Matsuyama, Ehime 790-8577, Japan

\begin{tabular}{|c|c|c|}
\hline S. No. & Table of Contents & Page No \\
\hline 1. & General Information & $2-3$ \\
\hline 2. & Synthetic Procedures and Compound Data & 3-4 \\
\hline 3. & High-Resolution ESI-TOF-MS and MALDI-TOF data & $5-7$ \\
\hline 4. & ${ }^{1} \mathrm{H},{ }^{1} \mathrm{H}^{-1} \mathrm{H}$ COSY and ${ }^{13} \mathrm{C}$ NMR spectra & 8-16 \\
\hline 5. & X-ray Crystallographic details and secondary $\mathrm{C}-\mathrm{H} \cdot \cdots \mathrm{N}$ interactions & 17-19 \\
\hline 6. & UV/Vis absorption spectra & 20-21 \\
\hline 7. & Cyclic Voltammetry & 22 \\
\hline 9. & $\begin{array}{l}\text { DFT Calculations: } \\
\text { a. Optimized Structures } \\
\text { b. NICS plots } \\
\text { c. Energy level diagrams and AICD plots } \\
\text { d. TD-DFT absorption spectra, Selected transitions and oscillator } \\
\text { strength data } \\
\text { f. Cartesian Coordinates }\end{array}$ & $\begin{array}{c}23 \\
24-25 \\
26-28\end{array}$ \\
\hline 10. & Supporting References & 49 \\
\hline
\end{tabular}




\section{General Information:}

The reagents and materials for the synthesis were used as obtained from Sigma - Aldrich chemical suppliers. All solvents were purified and dried by standard methods prior to use. Silica gel column chromatography was performed on Wakogel C-200 and C-300. Alumina column chromatography was performed on Active alumina (basic). Thin-layer chromatography (TLC) was carried out on aluminum sheets coated with silica gel 60 F254 (Merck 5554). Recrystallized samples of porphyrinoids were utilized for all the spectroscopic measurements. ${ }^{1} \mathrm{H}$ NMR $(500 \mathrm{MHz})$ and ${ }^{13} \mathrm{C}$ NMR (125 MHz) spectra were recorded on BRUKER Advance $500 \mathrm{MHz}$ spectrometer, and chemical shifts were reported as the delta scale in ppm relative to $\mathrm{CHCl}_{3}$ as an internal reference for ${ }^{1} \mathrm{H}(\delta=7.26 \mathrm{ppm})$ and for ${ }^{13} \mathrm{C}(\delta=77.0 \mathrm{ppm})$. The optical absorption spectra were recorded on a Shimadzu (Model UV-3600) spectrophotometer. Concentrations of solutions are $c a$. to be $1 \times 10^{-6} \mathrm{M}$ (porphyrin Soret band) and $5 \times 10^{-5} \mathrm{M}$ (porphyrin Q-bands). Cyclic and differential-pulse voltammetric measurements were performed on a PC-controlled electrochemical analyzer $(\mathrm{CH}$ instruments model CHI620C) using a conventional three-electrode cell for samples $(1 \mathrm{mM})$ dissolved in dry $\mathrm{CH}_{2} \mathrm{Cl}_{2}$ Containing $0.1 \mathrm{M} \mathrm{n}-\mathrm{Bu}_{4} \mathrm{NPF}_{6}\left(\mathrm{TBAPF}_{6}\right)$ as the supporting electrolyte. Measurements were carried out under an Argon atmosphere. A Glassy carbon working electrode, a calomel reference electrode and platinum wire counter electrode were used in all electrochemical experiments. The potentials were calibrated using the ferrocenium/ferrocene couple.

\section{DFT calculations}

All calculations were carried out using the Gaussian 16 C.01 program. ${ }^{[\mathrm{S} 1]}$ Calculations were performed by the density functional theory (DFT) method with restricted B3LYP (Becke's three-parameter hybrid exchange functionals and the Lee-Yang-Parr correlation functional $)^{[\mathrm{S} 2]}$ level, employing a basis sets 6-31G(d). Vertical electronic excitations based on B3LYP optimized geometries using the time-dependent density functional theory (TDDFT) formalism. ${ }^{[\mathrm{S} 3]}$ NICS values were calculated with GIAO method at the B3LYP level employing a basis sets 6-31G(d).

\section{X-ray crystallographic analysis}

Suitable crystals of 4T for X-ray analysis were obtained from slow evaporation of 1:1 benzonitrile and nitrobenzene at room temperature over a period of 7 days. X-ray diffraction analysis was performed using a Rigaku VariMax with Saturn diffractometer using multilayer 
mirror monochromated Mo $K \alpha$ radiation $(\lambda=0.71073 \AA)$ at $100 \pm 1 \mathrm{~K}$. The crystal was mounted in cryoloops. Data collection was performed using CrystalClear ${ }^{[\mathrm{S} 4]}$ software and data reduction was performed using CrysAlisPro ${ }^{[\mathrm{S} 5]}$ software. The data were corrected for Lorentz polarization and absorption effects. The structures were solved using SHELXT 2018/2 ${ }^{[\mathrm{S} 6]}$ and expanded using the Fourier technique. All calculations were performed using Olex2 $1.5^{[\mathrm{S} 7]}$ software. SHELXL-2018/3 ${ }^{[\mathrm{S} 8]}$ was used for structure refinement. The data were validated using PLATON. ${ }^{[\mathrm{S} 9]}$

\section{Synthetic Procedures and Compound Data}

Synthesis of $N$-(tert-Butoxycarbonyl)-pyrrole-2-boronic acid $\left(N\right.$-Boc-Py-B $\left.(\mathrm{OH})_{2}\right)$ was prepared by following the previously reported procedure. ${ }^{[\mathrm{S} 10]}$

Synthesis of (4-bromophenyl)(2,6-dimethylphenyl)methanol (2): To a $500 \mathrm{ml}$ two neck RB flask with magnetic stir bar, pre-activated Mg turnings (657 mg, $5.41 \mathrm{mmol}$ ) was added under inert atmosphere. A drop of 1,2-dibromoethane was added to activate the metal $\mathrm{Mg}$ surface followed by addition of $50 \mathrm{ml}$ of dry THF. 2-bromo-1,3-dimethylbenzene (4.3 $\mathrm{ml}, 27.02 \mathrm{mmol})$ was added dropwise for $30 \mathrm{~min}$ until the $\mathrm{Mg}$ disappears completely to give a yellowish brown 2,6-dimethylphenylmagnesium bromide solution. To this, a solution of 4-bromobenzaldehyde $1(1.0 \mathrm{~g}, 5.40 \mathrm{mmol})$ was added dropwise at $0{ }^{0} \mathrm{C}$ and stirred at room temperature for $2 \mathrm{~h}$. The reaction was quenched using saturated $\mathrm{NH}_{4} \mathrm{Cl}$ solution and the organic layer was extracted using $\mathrm{CH}_{2} \mathrm{Cl}_{2}$. The solvent was removed in vacuo and the crude product was purified via flash column chromatography on silica gel $\left(60 \% \mathrm{CH}_{2} \mathrm{Cl}_{2} / \mathrm{Hexane}\right)$ to obtain the desired product 2 as a light yellowish jelly in $73 \%$ yield $(1.14 \mathrm{~g}) .{ }^{1} \mathrm{H}$ NMR $\left(500 \mathrm{MHz}, \mathrm{CDCl}_{3}\right): \delta 7.42(\mathrm{~d}, J=8.5$ $\mathrm{Hz}, 2 \mathrm{H}), 7.14$ (dd, $J=11.8,7.9 \mathrm{~Hz}, 3 \mathrm{H}), 7.04$ (d, $J=7.5 \mathrm{~Hz}, 2 \mathrm{H}), 6.28$ (s, 1H), $2.26(\mathrm{~s}, 6 \mathrm{H})$. ${ }^{13} \mathrm{C} \mathrm{NMR}\left(126 \mathrm{MHz}, \mathrm{CDCl}_{3}\right): \delta 142.2,139.1,137.3,131.5,130.1,129.7,128.3,127.6,127.4$, 120.7, 71.0, 21.4, 20.9. HRMS (ESI ${ }^{+}$) m/z: 273.0272 (calcd. for $\mathrm{C}_{15} \mathrm{H}_{14} \mathrm{Br}[\mathrm{M}-\mathrm{OH}] 273.0273$ ), error in $\mathrm{ppm}=0.36$.

\section{Synthesis of (tert-butyl 2-(4-((2,6-dimethylphenyl)(hydroxy)methyl)phenyl)-1H-pyrrole-}

1-carboxylate) (4): A mixture of 2 (500 mg, $1.72 \mathrm{mmol}), \mathrm{N}$-(tert-butoxycarbonyl)-pyrrole-2boronic acid 3 (724 mg, $3.43 \mathrm{mmol}$ ), 2 $\mathrm{M} \mathrm{K}_{2} \mathrm{CO}_{3}$ (4.74 g, $\left.\left.34.34 \mathrm{mmol}\right), \mathrm{Pd}_{(} \mathrm{PPh}_{3}\right)_{4}(0.198 \mathrm{~g}$, $0.172 \mathrm{mmol}, 10 \mathrm{~mol} \%$ ) and was heated in an oil bath to $85^{\circ} \mathrm{C}$ in THF with stirring for $4 \mathrm{~h}$. The crude product was purified via flash column chromatography on silica gel $(40 \%$ $\left.\mathrm{CH}_{2} \mathrm{Cl}_{2} / \mathrm{Hexane}\right)$ to provide the title compound 4 in a $70 \%$ yield $(0.45 \mathrm{~g})$ as a stable off-white solid. ${ }^{1} \mathrm{H}$ NMR (500 MHz, $\left.\mathrm{CDCl}_{3}\right): \delta 7.35(\mathrm{~s}, 1 \mathrm{H}), 7.29(\mathrm{~d}, J=8.0 \mathrm{~Hz}, 2 \mathrm{H}), 7.26(\mathrm{~s}, 2 \mathrm{H}), 7.14$ (t, $J=7.5 \mathrm{~Hz}, 1 \mathrm{H}), 7.05(\mathrm{~d}, J=7.5 \mathrm{~Hz}, 2 \mathrm{H}), 6.38(\mathrm{~s}, 1 \mathrm{H}), 6.22(\mathrm{~s}, 1 \mathrm{H}), 6.18(\mathrm{~s}, 1 \mathrm{H}), 2.31$ (s, 
7H), $1.36(\mathrm{~s}, 9 \mathrm{H}) .{ }^{13} \mathrm{C} \mathrm{NMR}\left(126 \mathrm{MHz}, \mathrm{CDCl}_{3}\right): \delta 149.7,141.9,139.4,137.3,135.1,133.0$, $130.0,129.2,128.0,125.0,122.8,114.7,110.8,83.8, \quad 71.4,27.9,21.0$. HRMS $\left(\mathrm{ESI}^{+}\right) m / z: 360.1955$ (calcd. for $\mathrm{C}_{24} \mathrm{H}_{26} \mathrm{NO}_{2}[\mathrm{M}-\mathrm{OH}] 360.1958$ ), error in ppm $=0.83$.

Synthesis of (4-(1H-pyrrol-2-yl)phenyl)(2,6-dimethylphenyl)methanol (5): To a degassed solution of THF $(12 \mathrm{ml})$ in $25 \mathrm{ml}$ two neck round bottom flask, a solution of 4 (200 $\mathrm{mg}, 0.53$ mmol) was added at $0{ }^{\circ} \mathrm{C}$. $\mathrm{MeOH}(4 \mathrm{ml})$ followed by $\mathrm{NaOMe}(573 \mathrm{mg}, 10.6 \mathrm{mmol})$ was added and stirred for $3 \mathrm{~h}$. After standard aqueous work-up followed by the removal of solvent under vacuum afforded colourless 5 in a quantitative yield. ${ }^{1} \mathrm{H}$ NMR $\left(500 \mathrm{MHz}, \mathrm{CDCl}_{3}\right): \delta 8.46(\mathrm{~s}$, $1 \mathrm{H}, \mathrm{NH}), 7.41(\mathrm{~d}, J=8.3 \mathrm{~Hz}, 2 \mathrm{H}), 7.28(\mathrm{~s}, 1 \mathrm{H}), 7.16$ (d, $J=7.6 \mathrm{~Hz}, 1 \mathrm{H}), 7.06$ (d, $J=7.5 \mathrm{~Hz}$, $2 \mathrm{H}), 6.84(\mathrm{~s}, 1 \mathrm{H}), 6.51(\mathrm{~d}, J=2.3 \mathrm{~Hz}, 1 \mathrm{H}), 6.38-6.25(\mathrm{~m}, 2 \mathrm{H}), 2.30(\mathrm{~s}, 6 \mathrm{H}) .{ }^{13} \mathrm{C} \mathrm{NMR}(126$ $\left.\mathrm{MHz}, \mathrm{CDCl}_{3}\right): \delta 141.0,139.5,137.4,132.0,131.4,129.6,128.1,126.3,123.9,119.0,118.8$, 110.2, 106.0, 71.3, 68.2, 25.8, 20.9. HRMS (ESI $\left.{ }^{+}\right) m / z: 260.1434$ (calcd. for $\mathrm{C}_{19} \mathrm{H}_{18} \mathrm{~N}$ [M-OH] 260.1434), error in ppm $=0.00$.

Synthesis of Cyclotrimer (3T) and Cyclotetramer (4T): Precursor 5 (200 mg, $0.72 \mathrm{mmol}$ ) was added to a oven-dried $1000 \mathrm{~mL}$ single neck $\mathrm{RB}$ containing $500 \mathrm{ml}$ dry $\mathrm{CH}_{2} \mathrm{Cl}_{2}$ fitted with Ar-bubbling. $\mathrm{BF}_{3} \cdot \mathrm{OEt}_{2}(66.79 \mu \mathrm{L}, 0.541 \mathrm{mmol})$ was then added to it and stirred in room temperature for $2 \mathrm{~h}$ in the absence of light under Argon atmosphere followed by the addition of few drops of $\mathrm{Et}_{3} \mathrm{~N}$. After complete consumption of the starting material, oxidation was accomplished using DDQ (164 mg, $0.72 \mathrm{mmol})$. The crude reaction mixture was purified via silica gel column chromatography, wherein 3T was obtained as yellowish orange and 4T as reddish orange solids in $4(22 \mathrm{mg})$ and $5 \%(37 \mathrm{mg})$ respectively. Although the yield of these macrocyclic products are low but the reaction is found to be reproducible.

Cyclotrimer (3T): ${ }^{1} \mathrm{H}$ NMR $\left(500 \mathrm{MHz}, \mathrm{CDCl}_{3}\right) \delta 8.13(\mathrm{~d}, J=8.1 \mathrm{~Hz}, 6 \mathrm{H}), 8.04(\mathrm{~d}, J=8.0 \mathrm{~Hz}$, $6 \mathrm{H}), 7.24$ (d, $J=7.6 \mathrm{~Hz}, 3 \mathrm{H}), 7.14(\mathrm{~d}, J=7.6 \mathrm{~Hz}, 6 \mathrm{H}), 6.82$ (d, $J=4.5 \mathrm{~Hz}, 3 \mathrm{H}), 6.75$ (d, $J=$ $4.6 \mathrm{~Hz}, 3 \mathrm{H}), 2.20$ (s, 18H). UV-Vis/NIR $\left(\mathrm{CH}_{2} \mathrm{Cl}_{2}\right): \lambda_{\max }, \mathrm{nm}\left(\varepsilon\left[\mathrm{x} 10^{5} \mathrm{M}^{-1} \cdot \mathrm{cm}^{-1}\right]\right): 367(0.429)$. HRMS (ESI ${ }^{+}$) m/z: 772.3681 (calcd. for $\mathrm{C}_{57} \mathrm{H}_{46} \mathrm{~N}_{3}\left[\mathrm{M}+\mathrm{H}^{+}\right]$772.3692).

Cyclotetramer (4T): ${ }^{1} \mathrm{H}$ NMR $(500 \mathrm{MHz}, \mathrm{CDCl} 3) \delta 8.69$ (s, 8H), 8.51 (s, 8H), $7.23(\mathrm{~s}, 4 \mathrm{H})$, $7.13(\mathrm{~d}, \mathrm{~J}=7.5 \mathrm{~Hz}, 8 \mathrm{H}), 6.94(\mathrm{~d}, \mathrm{~J}=4.3 \mathrm{~Hz}, 4 \mathrm{H}), 6.62$ (d, J = 4.3 Hz, 4H), 2.13 (s, 24H). UVVis/NIR $\left(\mathrm{CH}_{2} \mathrm{Cl}_{2}\right): \lambda_{\max }, \mathrm{nm}\left(\varepsilon\left[\mathrm{x} 10^{5} \mathrm{M}^{-1} . \mathrm{cm}^{-1}\right]\right): 429(0.175)$. HRMS $\left(\mathrm{ESI}^{+}\right) \mathrm{m} / z: 1029.4893$ (calcd. for $\mathrm{C}_{76} \mathrm{H}_{61} \mathrm{~N}_{4}\left[\mathrm{M}+\mathrm{H}^{+}\right]$1029.4896). 
3. High-Resolution ESI-TOF-MS and MALDI-TOF data

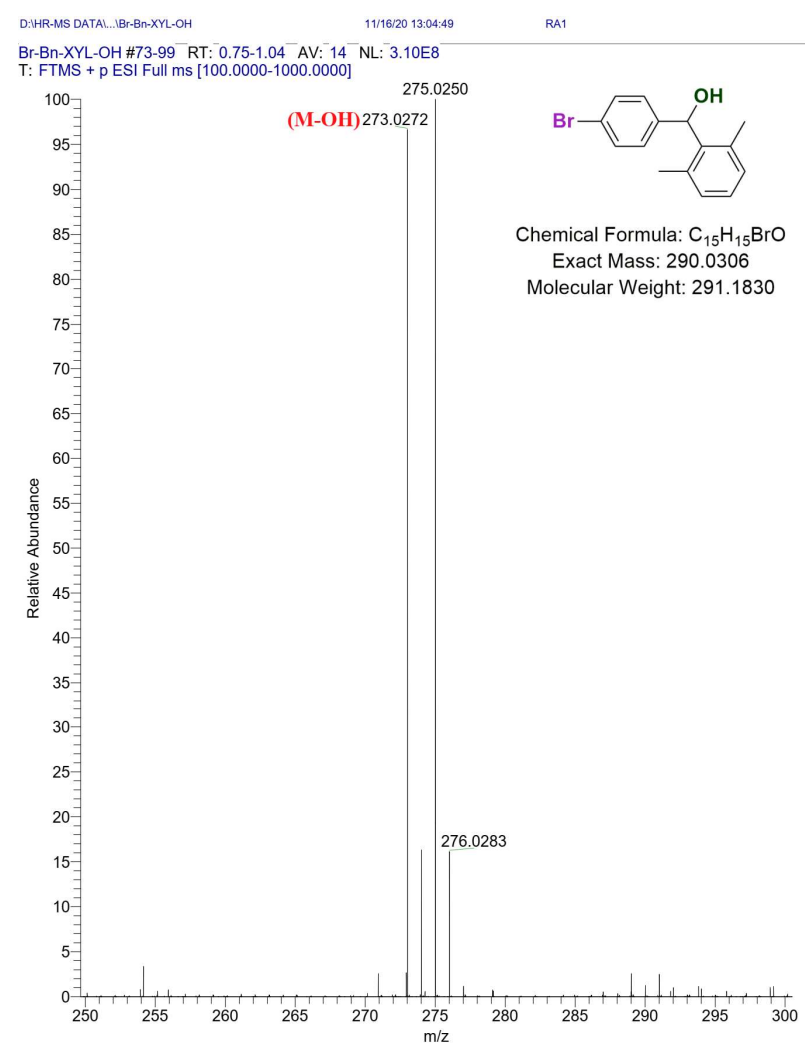

Figure S3-1: HR-MS spectrum of 2

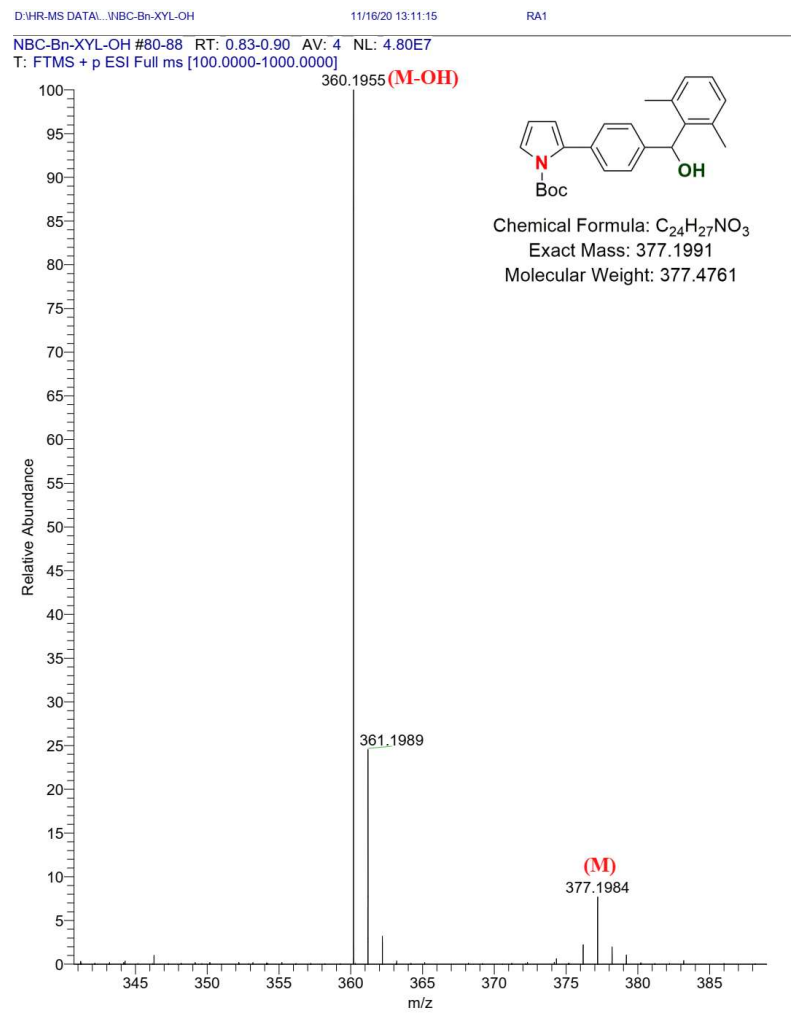

Figure S3-2: HR-MS spectrum of 4 


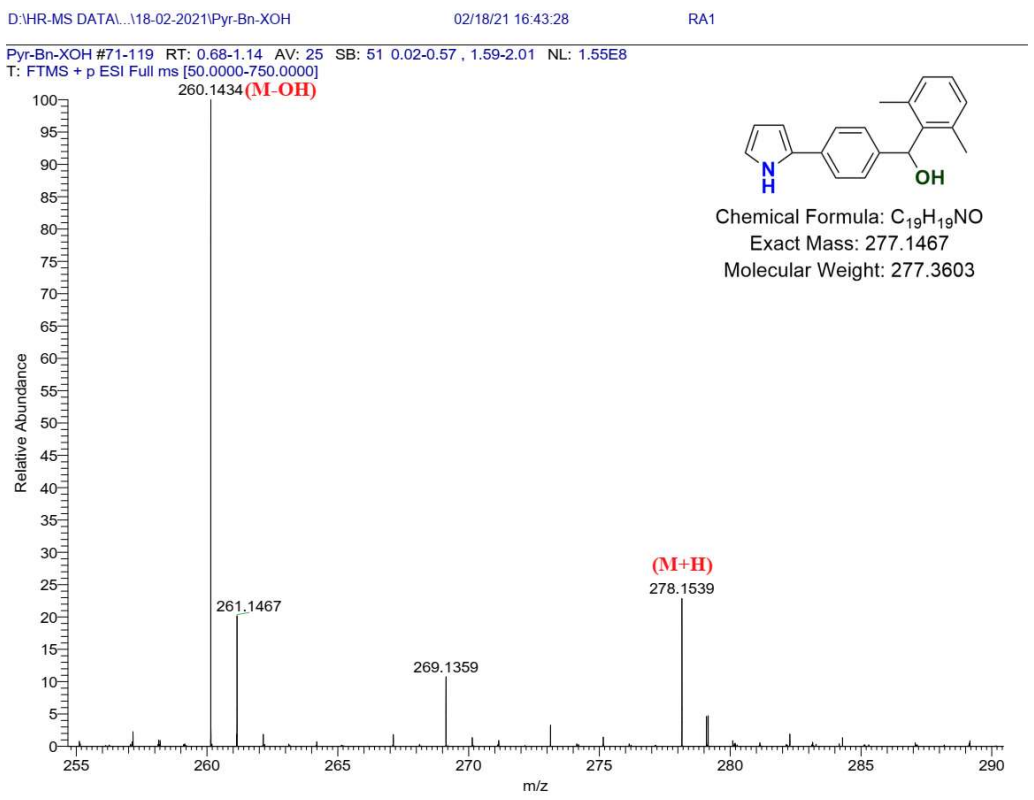

Figure S3-3: HR-MS spectrum of 5

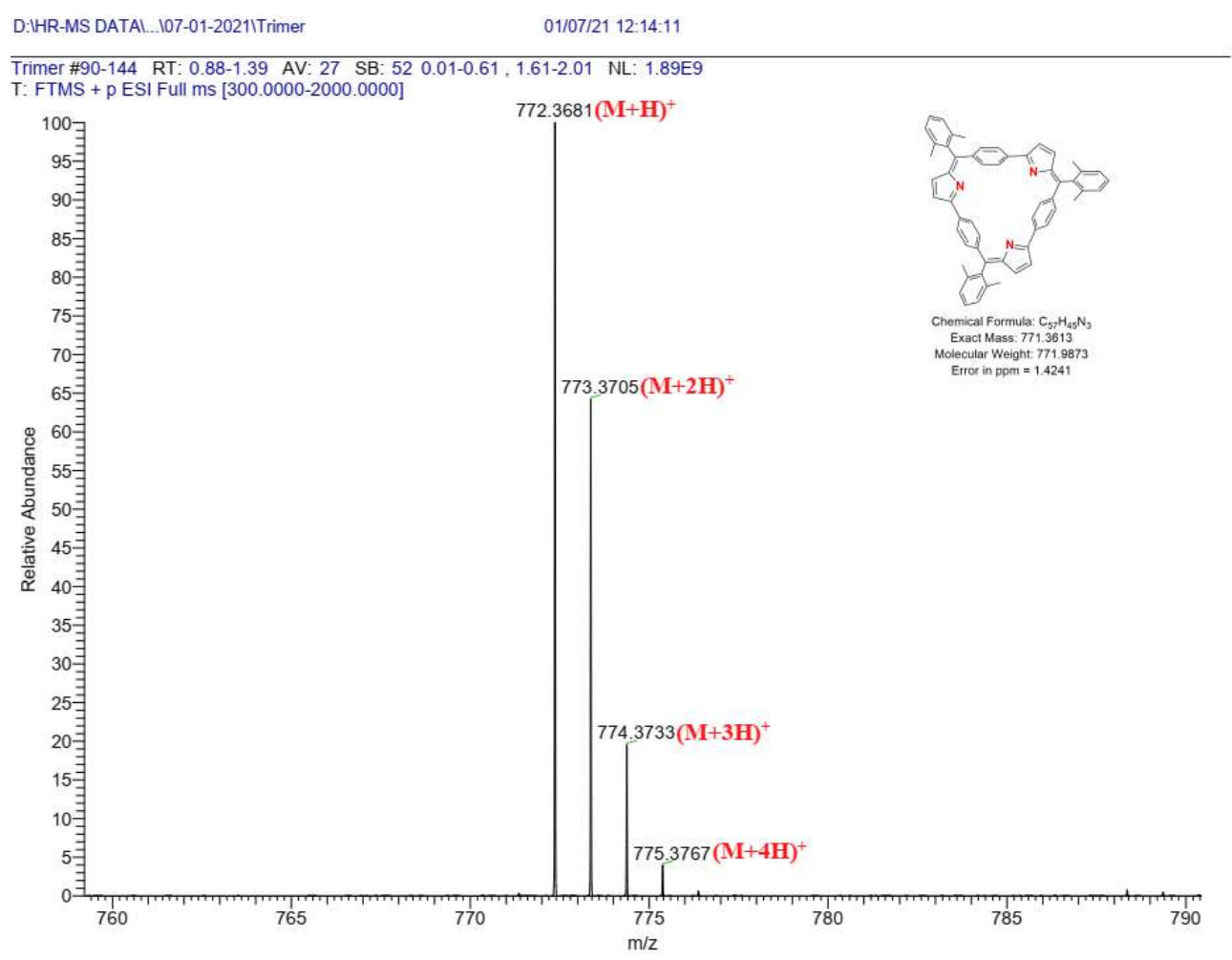

Figure S3-4: HR-MS spectrum of 3T 


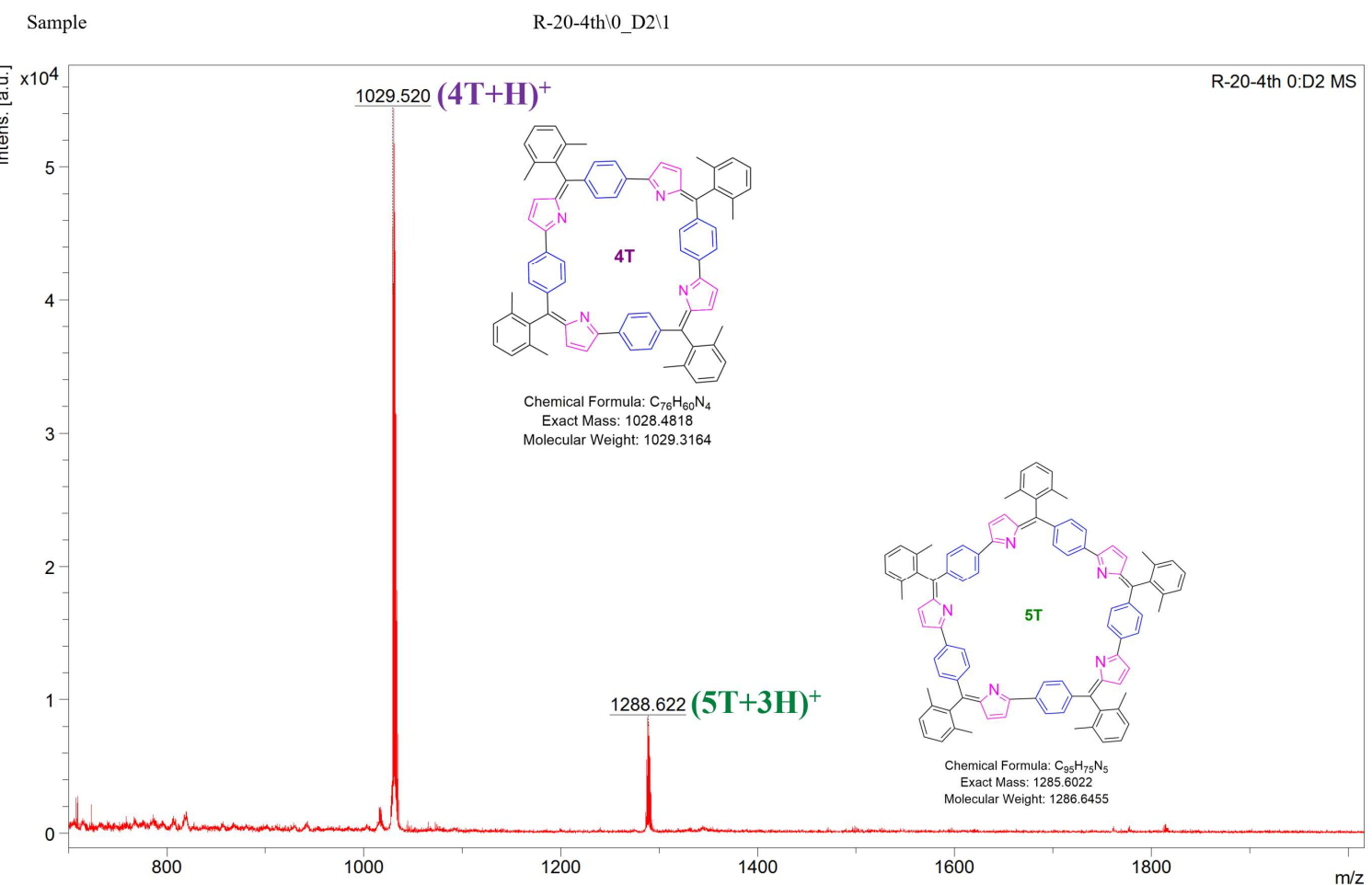

Figure S3-5: MALDI-TOF spectrum of reaction mixture $(4 \mathrm{~T}+5 \mathrm{~T})$

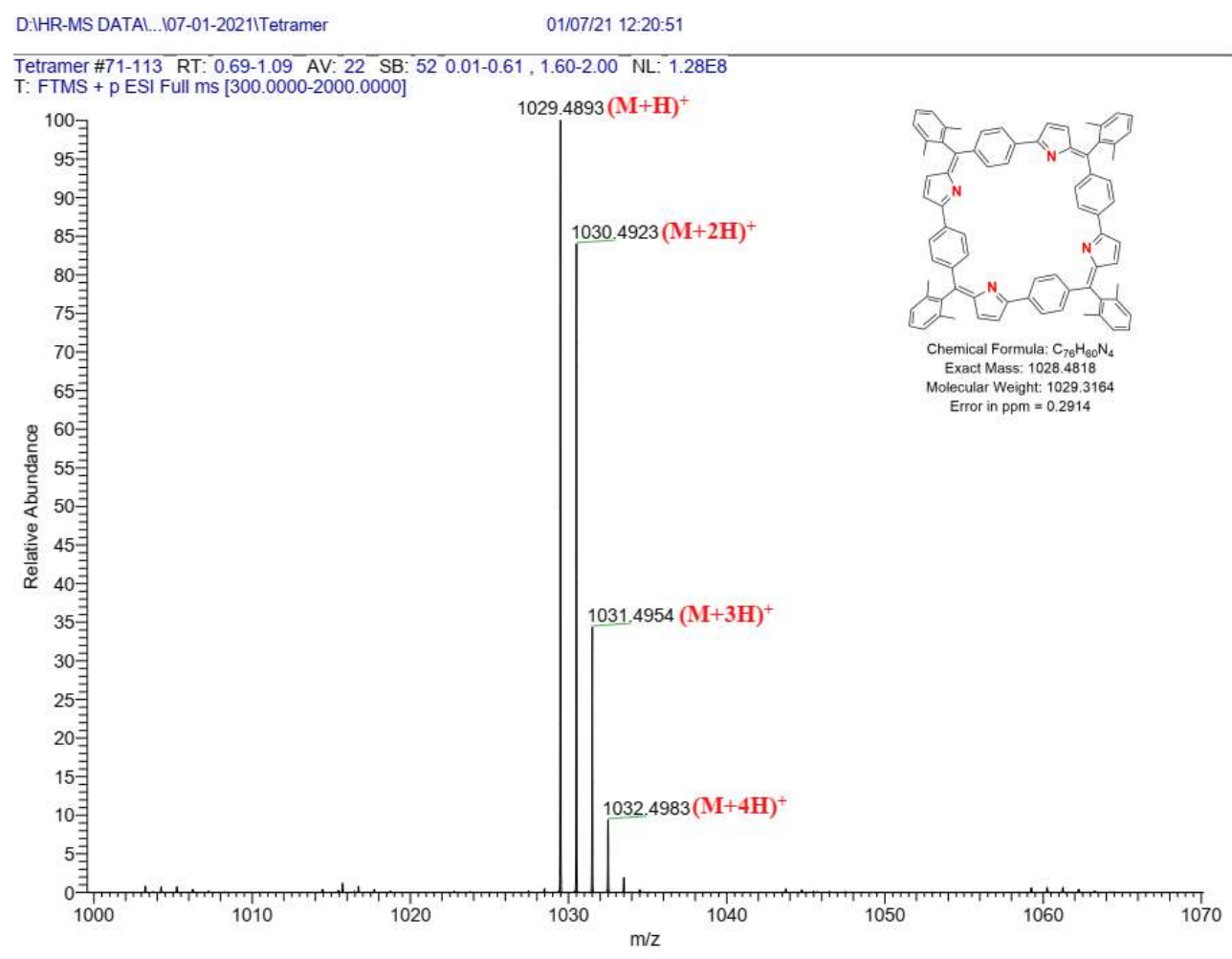

Figure S3-6: HR-MS spectrum of 4T 

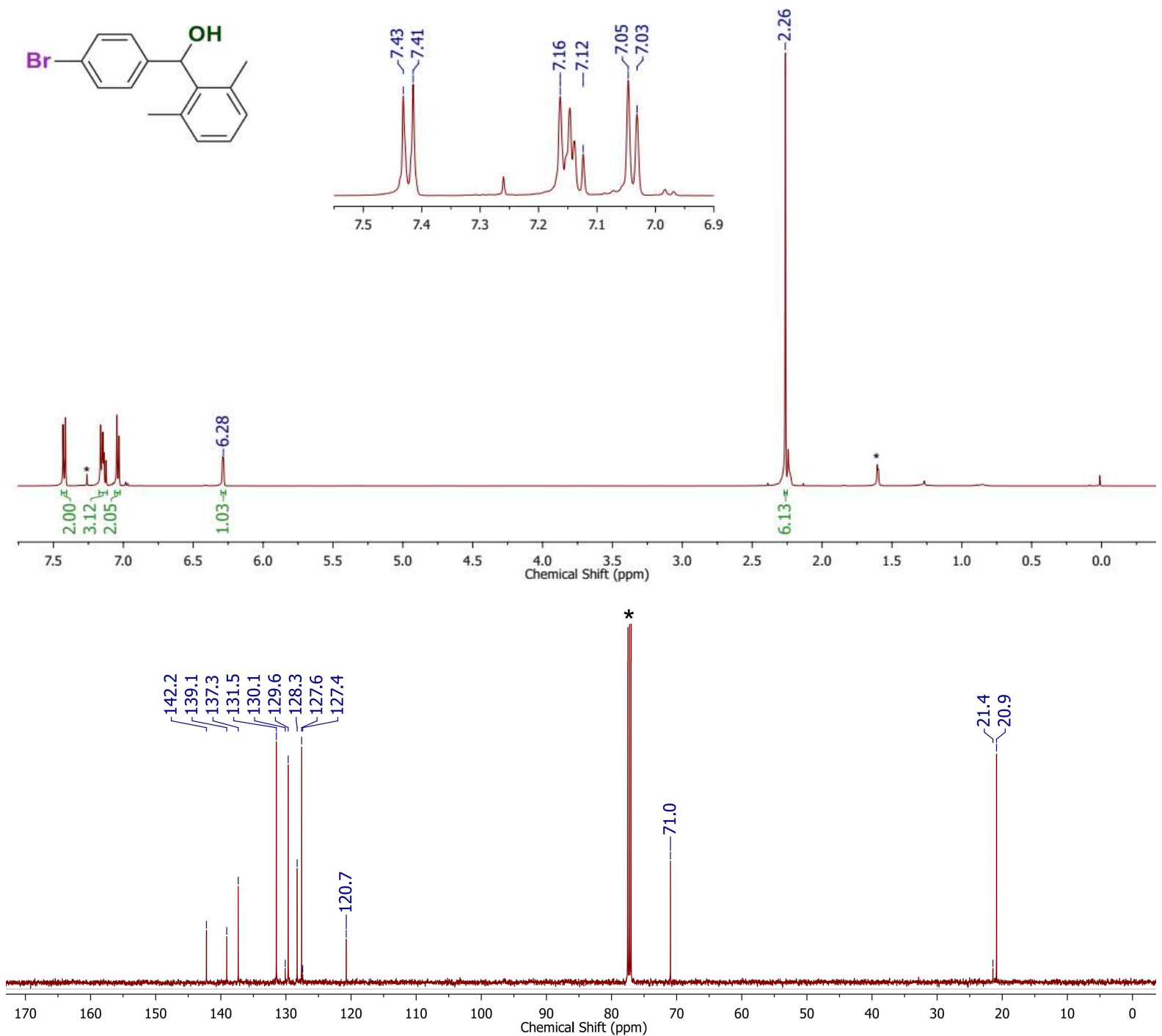

Figure S4-1: ${ }^{1} \mathrm{H}$ (top) and ${ }^{13} \mathrm{C}$ (bottom) NMR spectra of 2 in $\mathrm{CDCl}_{3}$ at $298 \mathrm{~K}(500 \mathrm{MHz})$. Signals marked with $(*)$ denote residual solvents or impurities. 

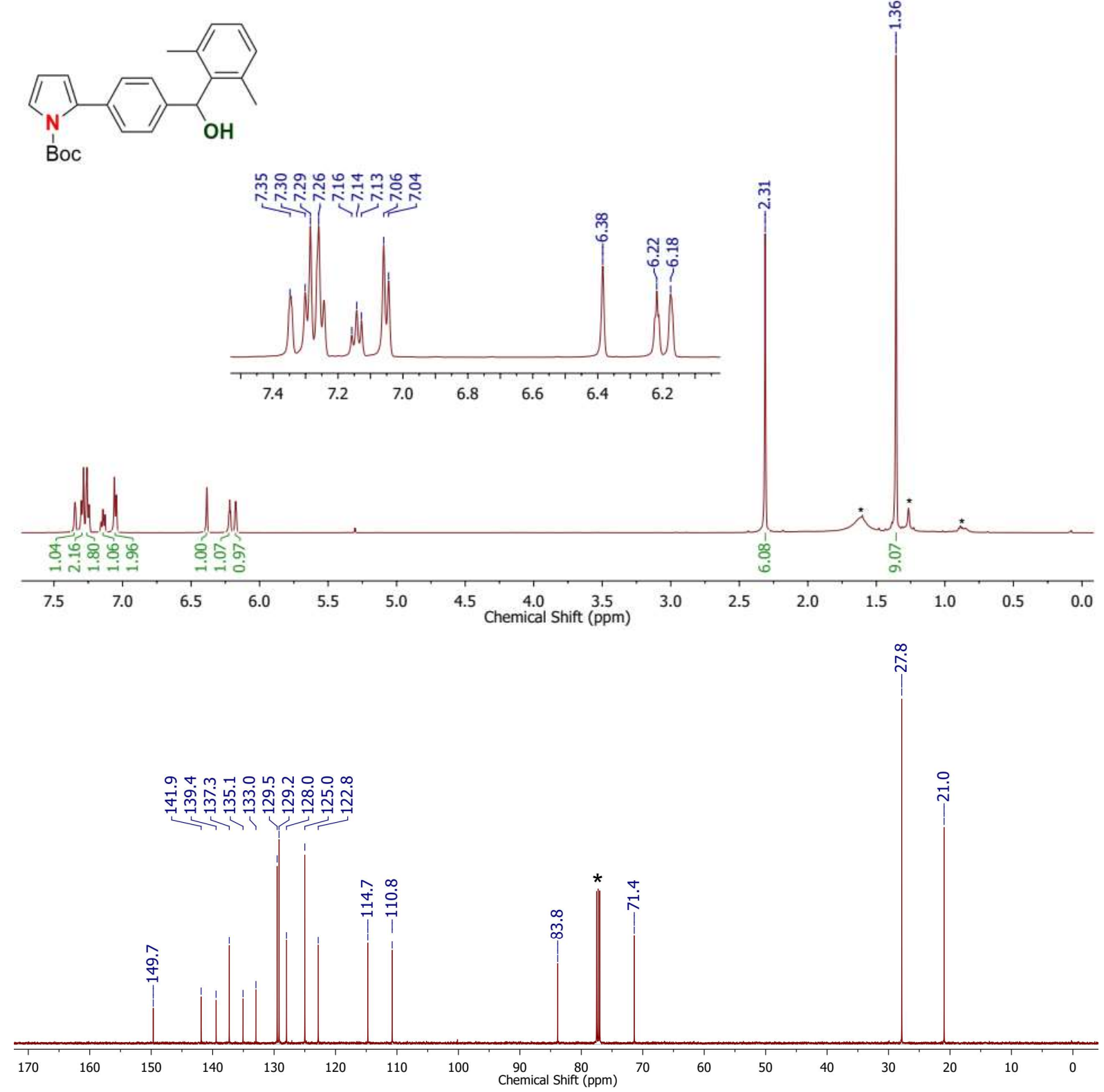

Figure S4-2: ${ }^{1} \mathrm{H}$ (top) and ${ }^{13} \mathrm{C}$ (bottom) NMR Spectra of 4 in $\mathrm{CDCl}_{3}$ at $298 \mathrm{~K}(500 \mathrm{MHz})$. Signals marked with $(*)$ denote residual solvents or impurities. 

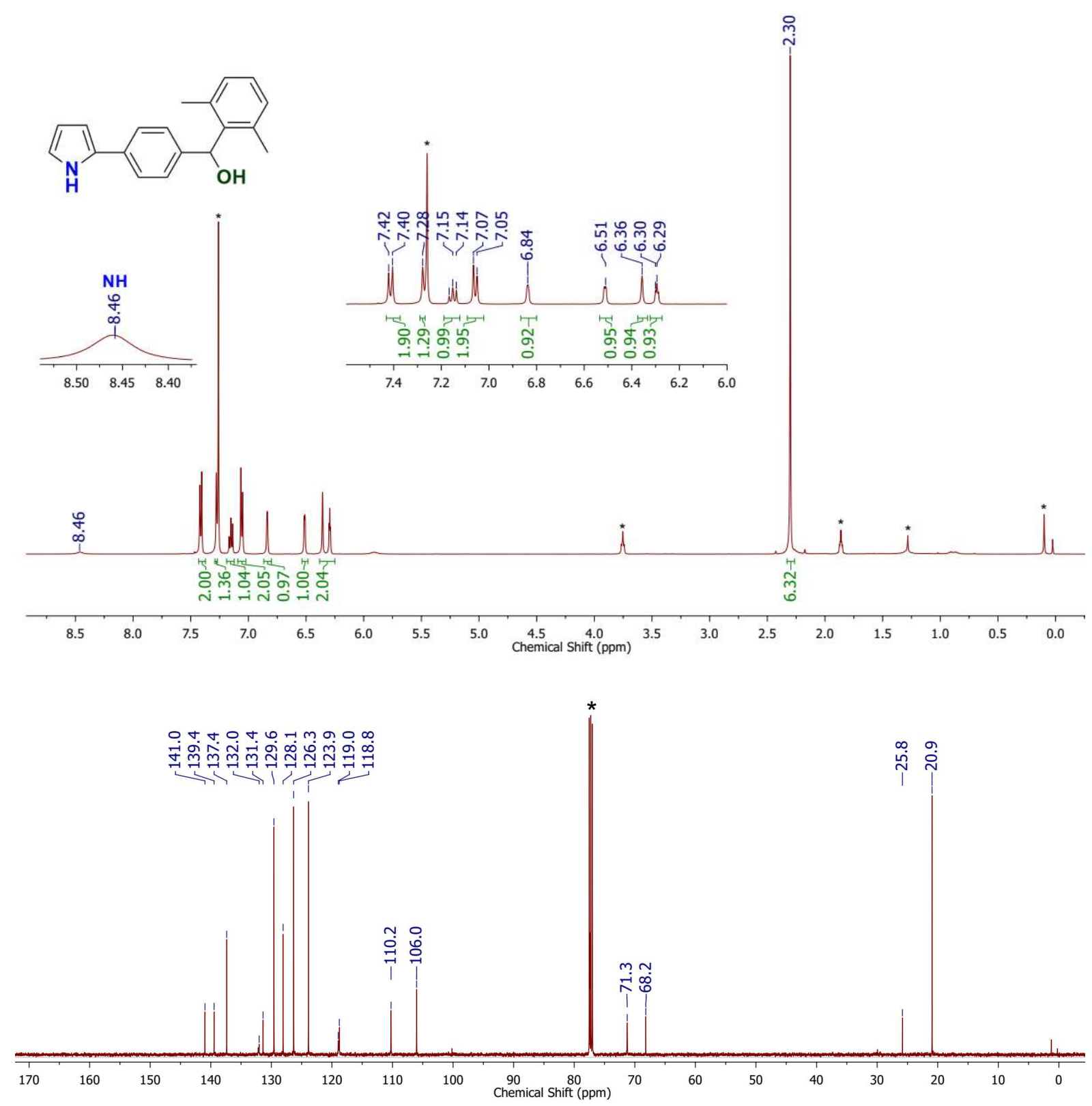

Figure S4-3: ${ }^{1} \mathrm{H}$ (top) and ${ }^{13} \mathrm{C}$ (bottom) NMR spectra of 5 in $\mathrm{CDCl}_{3}$ at $298 \mathrm{~K}(500 \mathrm{MHz})$. Signals marked with $(*)$ denote residual solvents or impurities. 


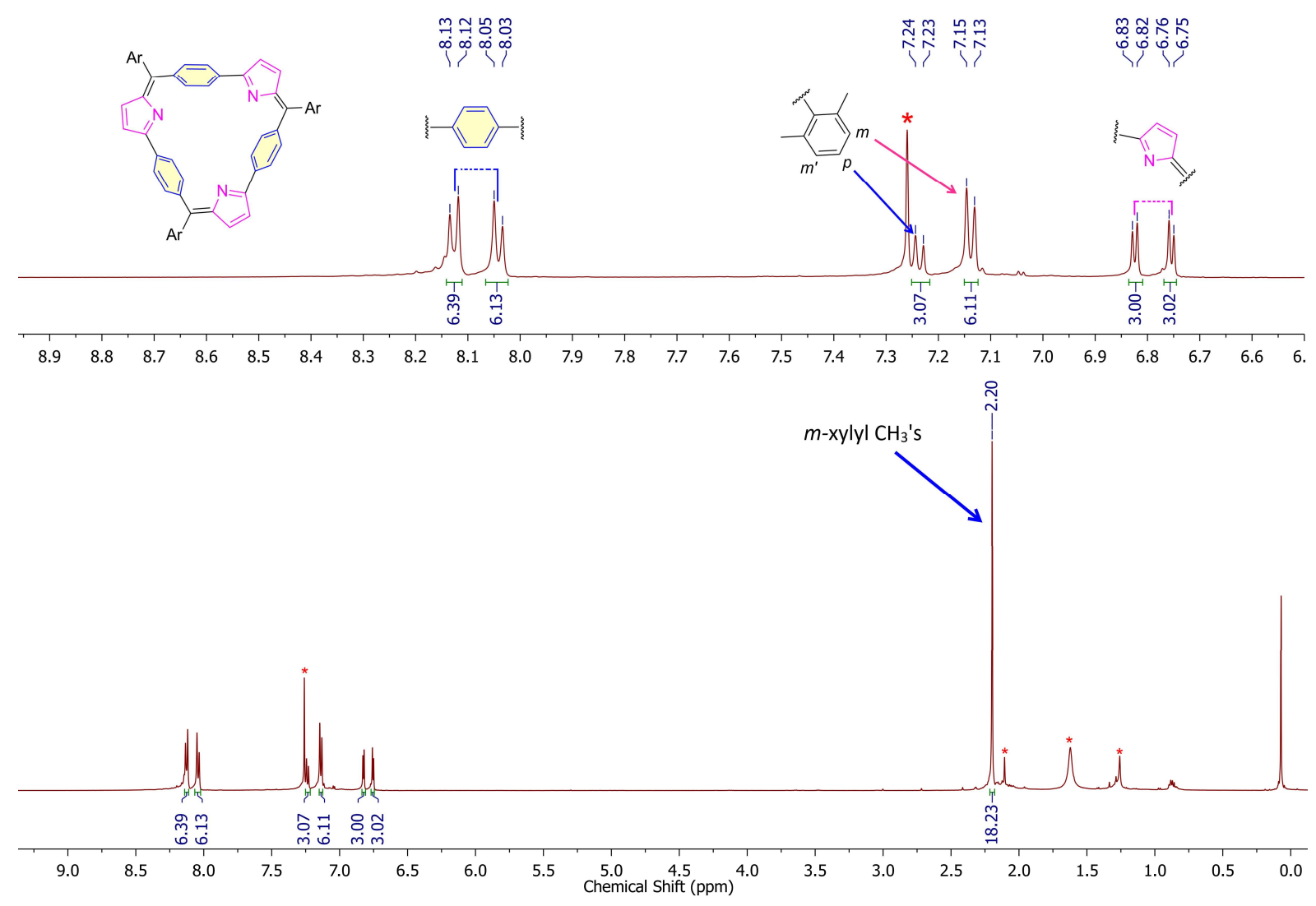

Figure S4-4: ${ }^{1} \mathrm{H}$ NMR spectra of $\mathbf{3 T}$ in $\mathrm{CDCl}_{3}$ at $298 \mathrm{~K}(500 \mathrm{MHz})$. Signals marked with (*) denote residual solvents or impurities.

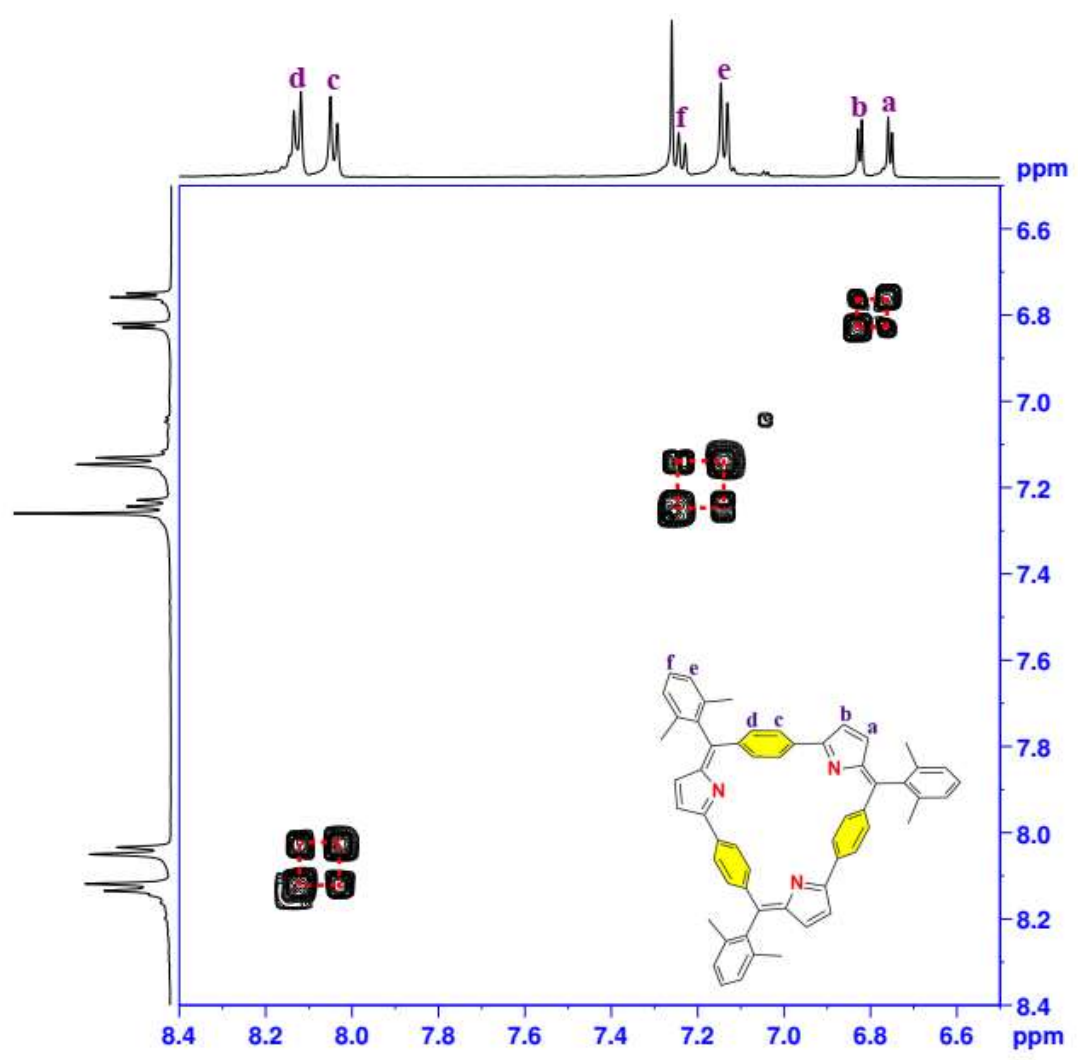

Figure S4-5: ${ }^{1} \mathrm{H}-{ }^{1} \mathrm{H}$ COSY spectrum of $\mathbf{3 T}$ in $\mathrm{CDCl}_{3}$ at $298 \mathrm{~K}(500 \mathrm{MHz})$. 


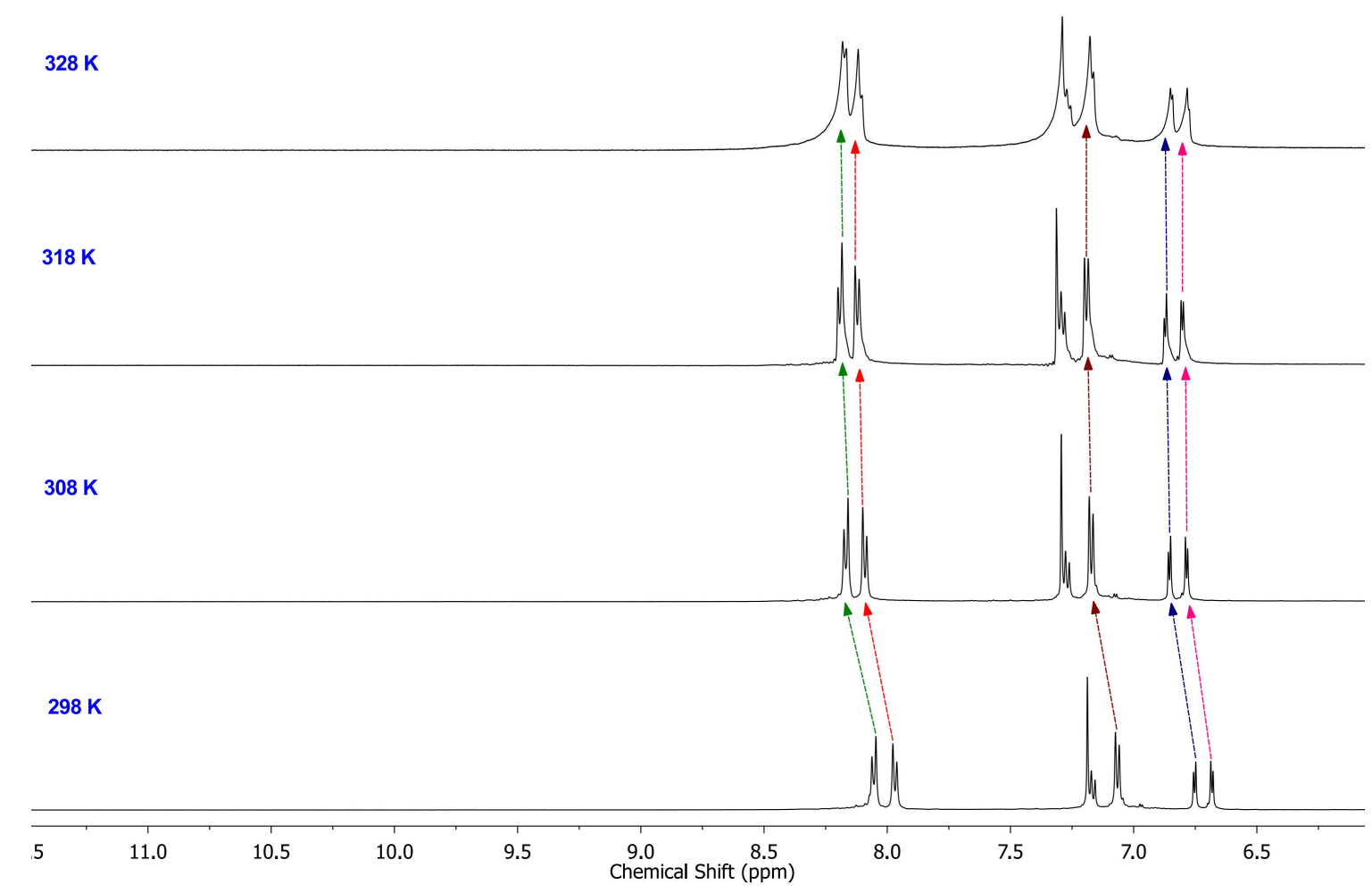

Figure S4-6: VT ${ }^{1} \mathrm{H}$ NMR spectra of 3T recorded in $\mathrm{CDCl}_{3}$ from 298 to $328 \mathrm{~K}(500 \mathrm{MHz})$.

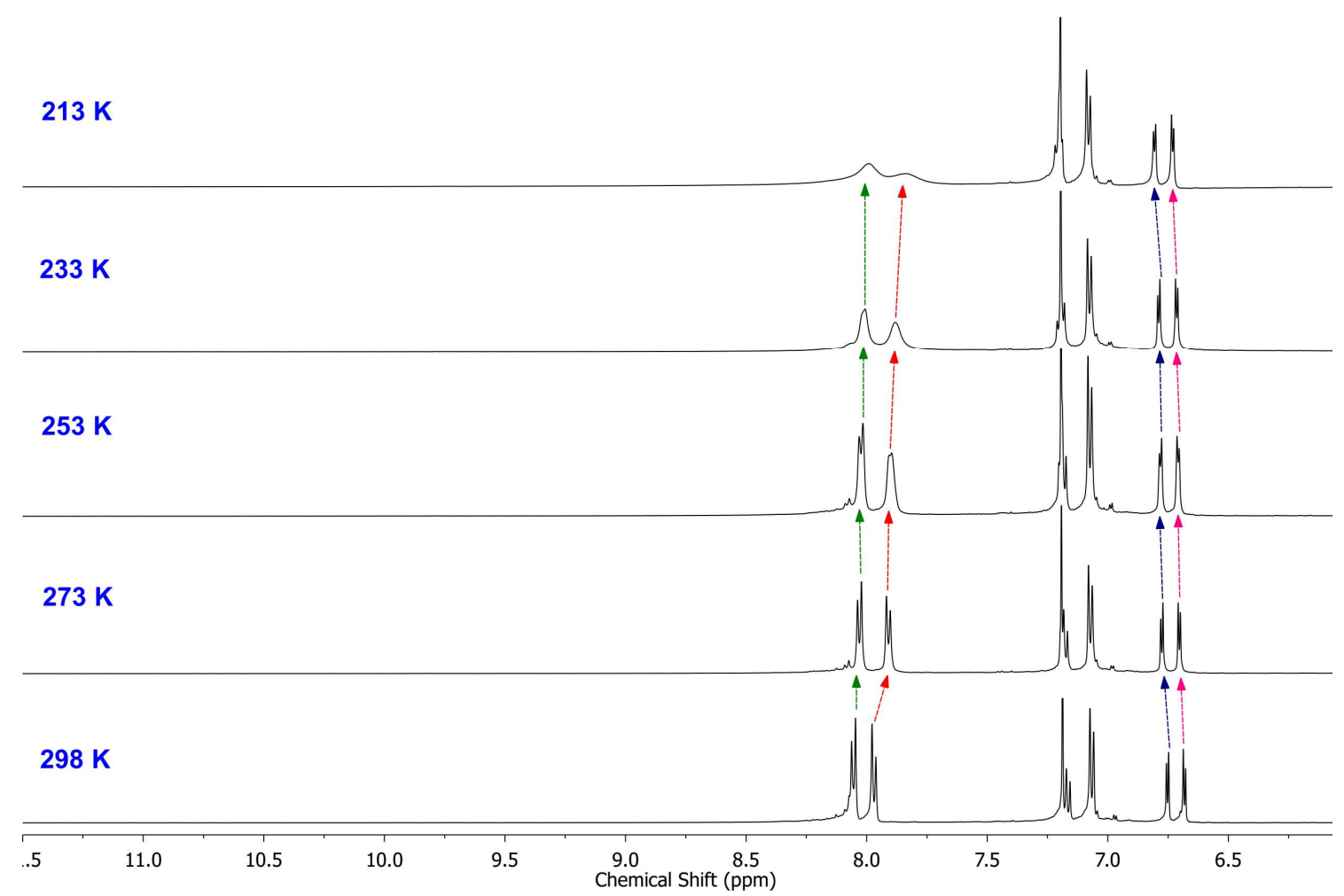

Figure S4-7: VT ${ }^{1} \mathrm{H}$ NMR spectra of 3 T recorded in $\mathrm{CDCl}_{3}$ from 298 to $213 \mathrm{~K}(500 \mathrm{MHz})$. 


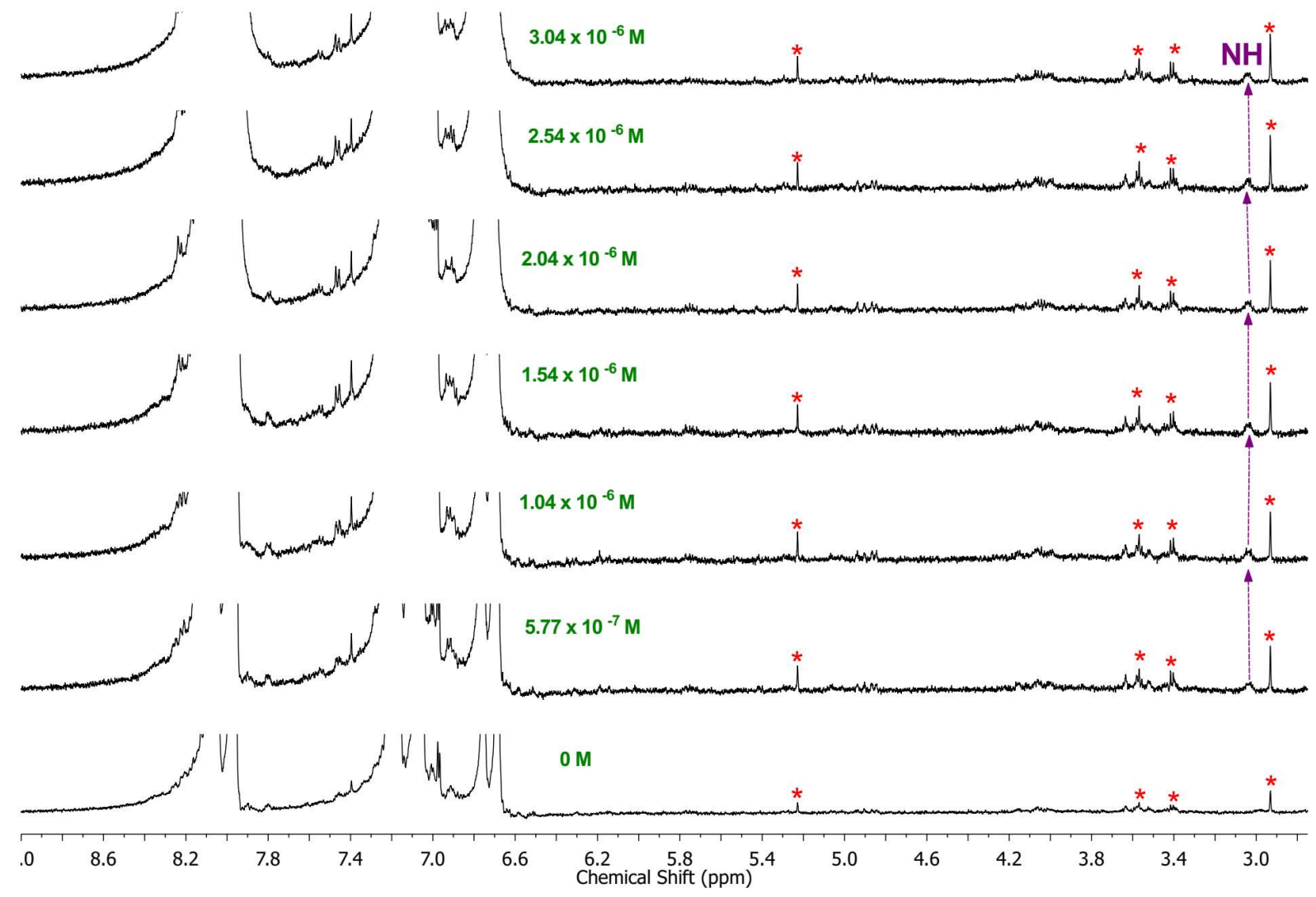

Figure S4-8: Partial ${ }^{1} \mathrm{H}$ NMR spectral titration of 3T recorded in $\mathrm{CDCl}_{3}$ with $\mathrm{CF}_{3} \mathrm{COOH}$ at $298 \mathrm{~K}(500 \mathrm{MHz})$.

$[\mathbf{3 T}]=6.17 \times 10^{-3} \mathrm{M},\left[\mathrm{CF}_{3} \mathrm{COOH}\right]=0-3.04 \times 10^{-6} \mathrm{M}\left(5.77 \times 10^{-7} \mathrm{M}=0.08\right.$ equiv., $1.04 \times 10^{-}$ ${ }^{6} \mathrm{M}=0.16$ equiv., $1.54 \times 10^{-6} \mathrm{M}=0.25$ equiv., $2.04 \times 10^{-6} \mathrm{M}=0.33$ equiv., $2.54 \times 10^{-6} \mathrm{M}=$ 0.41 equiv. and $3.04 \times 10^{-6} \mathrm{M}=0.5$ equiv.) 

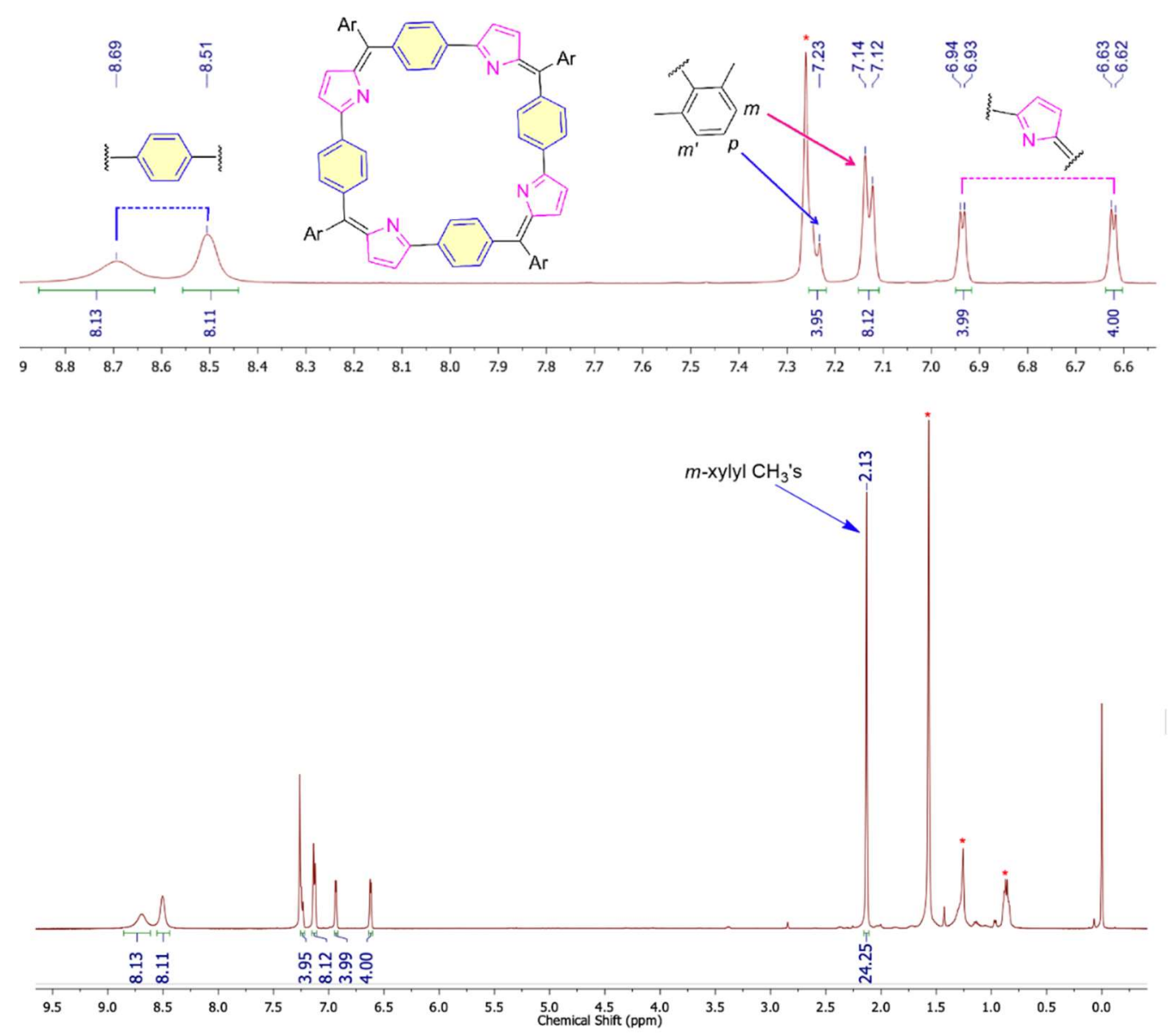

Figure S4-9: ${ }^{1} \mathrm{H}$ NMR spectra of $4 \mathrm{~T}$ in $\mathrm{CDCl}_{3}$ at $298 \mathrm{~K}(500 \mathrm{MHz})$. Signals marked with (*) denote residual solvents or impurities.

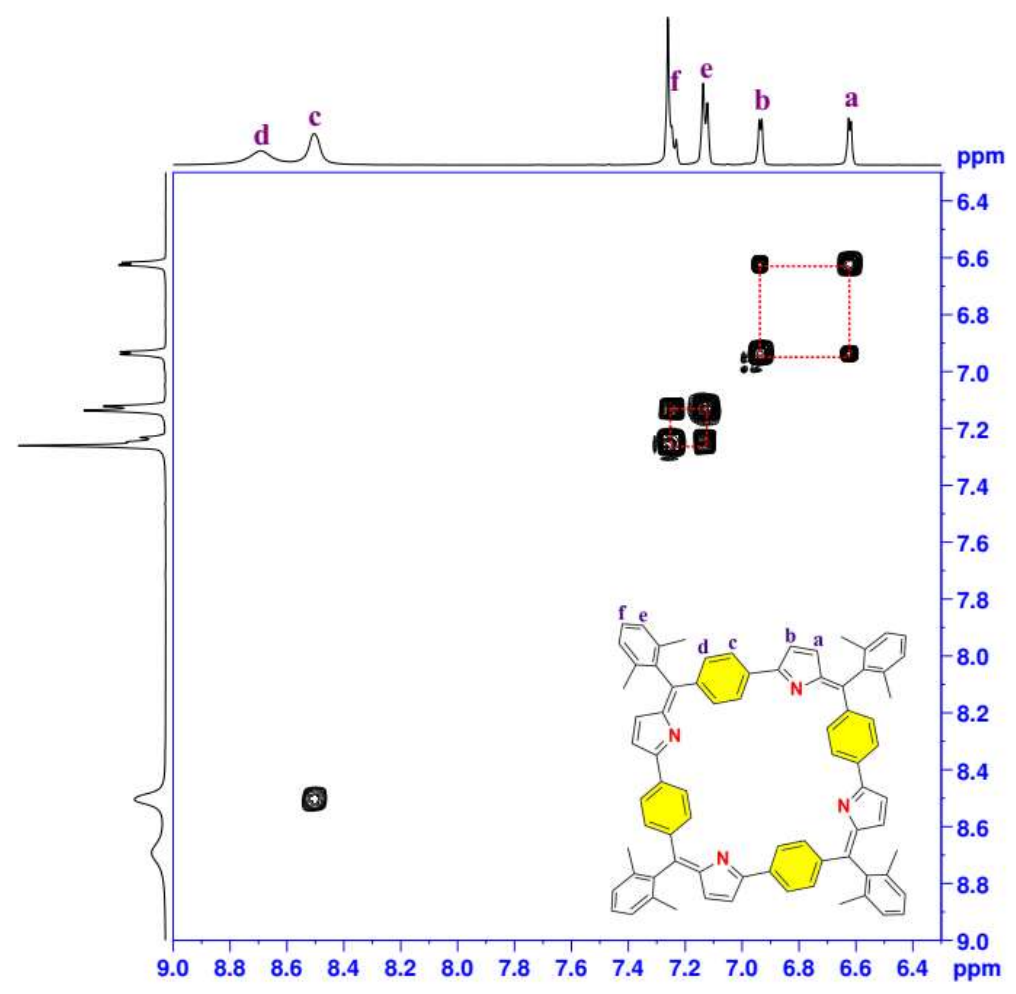

Figure S4-10: ${ }^{1} \mathrm{H}-{ }^{1} \mathrm{H}$ COSY Spectrum of $4 \mathrm{~T}$ in $\mathrm{CDCl}_{3}$ at $298 \mathrm{~K}(500 \mathrm{MHz})$. 


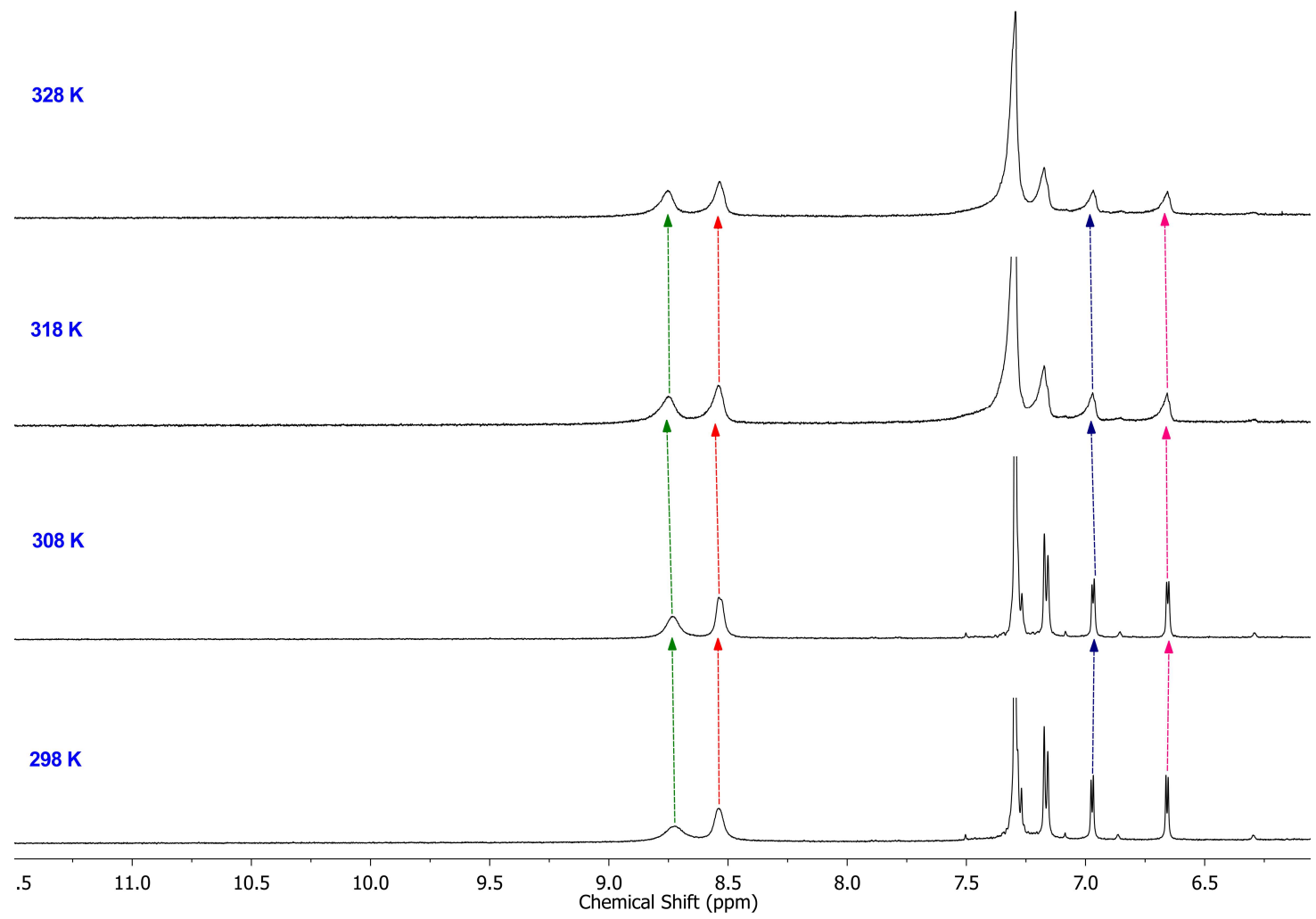

Figure S4-11: VT ${ }^{1} \mathrm{H}$ NMR spectra of 4 T recorded in $\mathrm{CDCl}_{3}$ from 298 to $328 \mathrm{~K}(500 \mathrm{MHz})$.

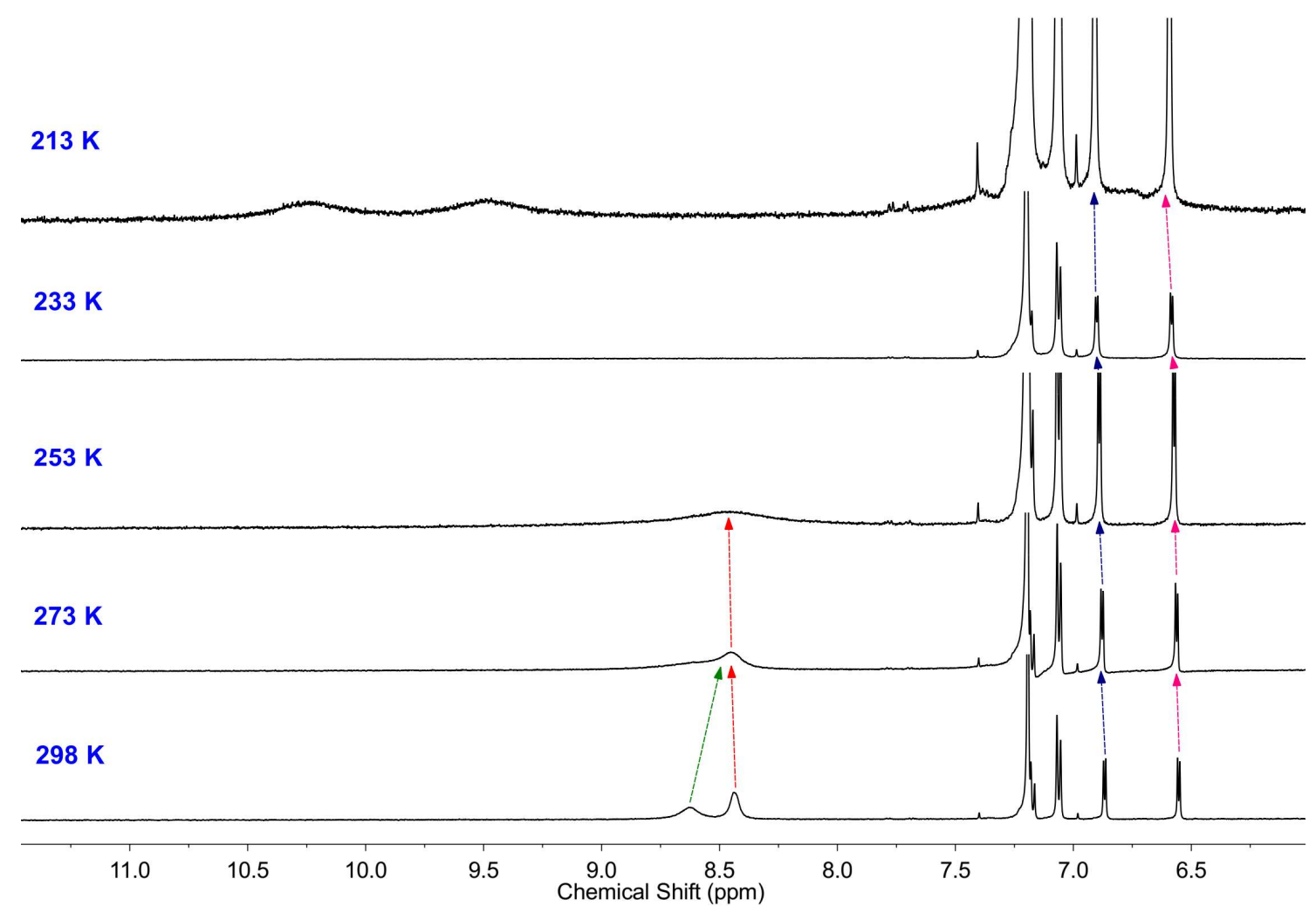

Figure S4-12: VT ${ }^{1} \mathrm{H}$ NMR spectra of 4T recorded in $\mathrm{CDCl}_{3}$ from 298 to $213 \mathrm{~K}(500 \mathrm{MHz})$. 


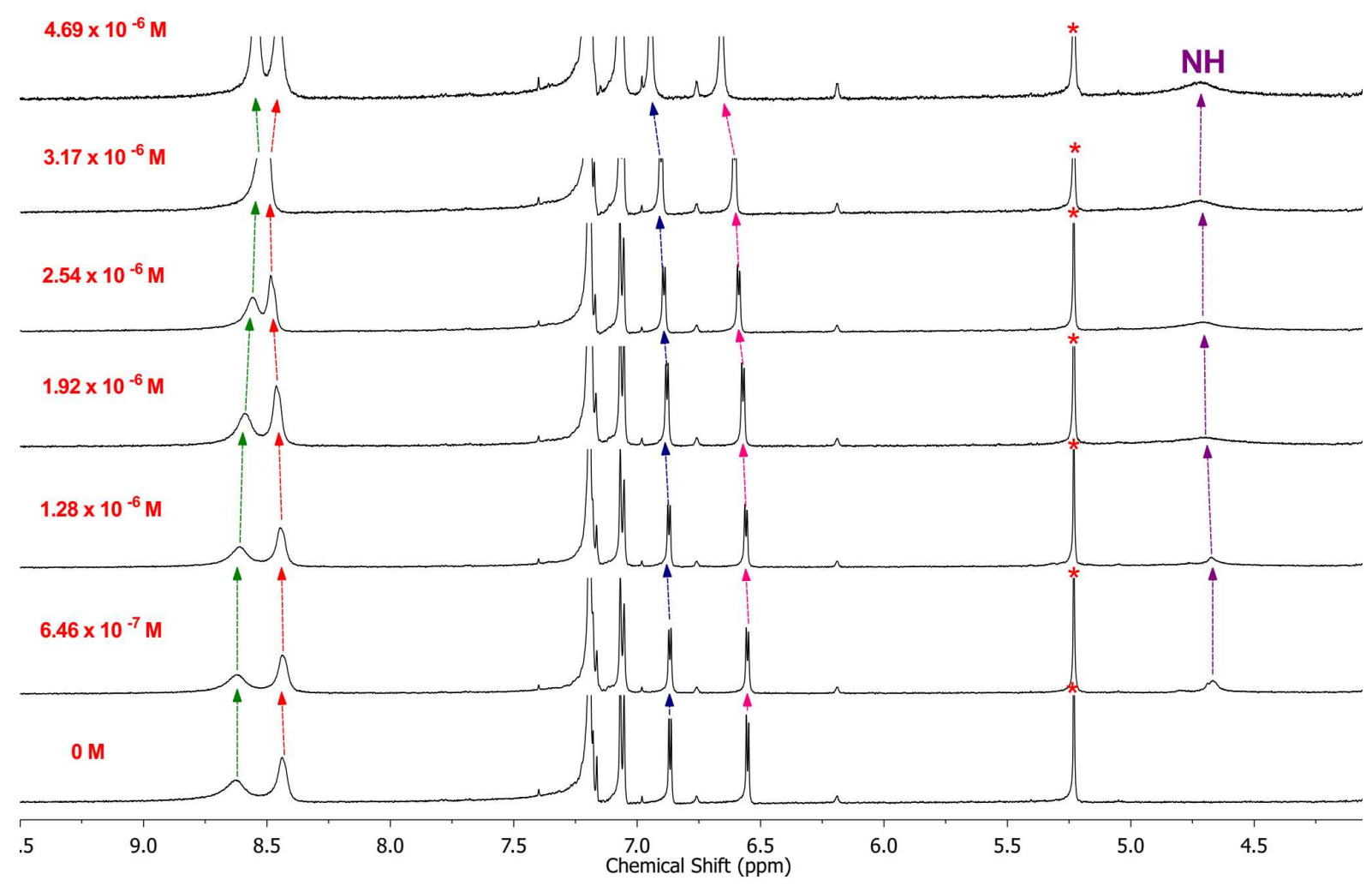

Figure S4-13: Partial ${ }^{1} \mathrm{H}$ NMR spectral titration of $4 \mathrm{~T}$ recorded in $\mathrm{CDCl}_{3}$ with $\mathrm{CF}_{3} \mathrm{COOH}$ at $298 \mathrm{~K}(500 \mathrm{MHz})$.

$[4 \mathrm{~T}]=5.62 \times 10^{-3} \mathrm{M},\left[\mathrm{CF}_{3} \mathrm{COOH}\right]=0-3.04 \times 10^{-6} \mathrm{M}\left(6.46 \times 10^{-7} \mathrm{M}=0.08\right.$ equiv., $1.28 \times 10^{-}$ ${ }^{6} \mathrm{M}=0.16$ equiv., $1.92 \times 10^{-6} \mathrm{M}=0.25$ equiv., $2.54 \times 10^{-6} \mathrm{M}=0.33$ equiv., $3.17 \times 10^{-6} \mathrm{M}=$ 0.41 equiv. and $4.69 \times 10^{-6} \mathrm{M}=0.62$ equiv.) 


\section{X-ray Crystallographic Data for 4T}
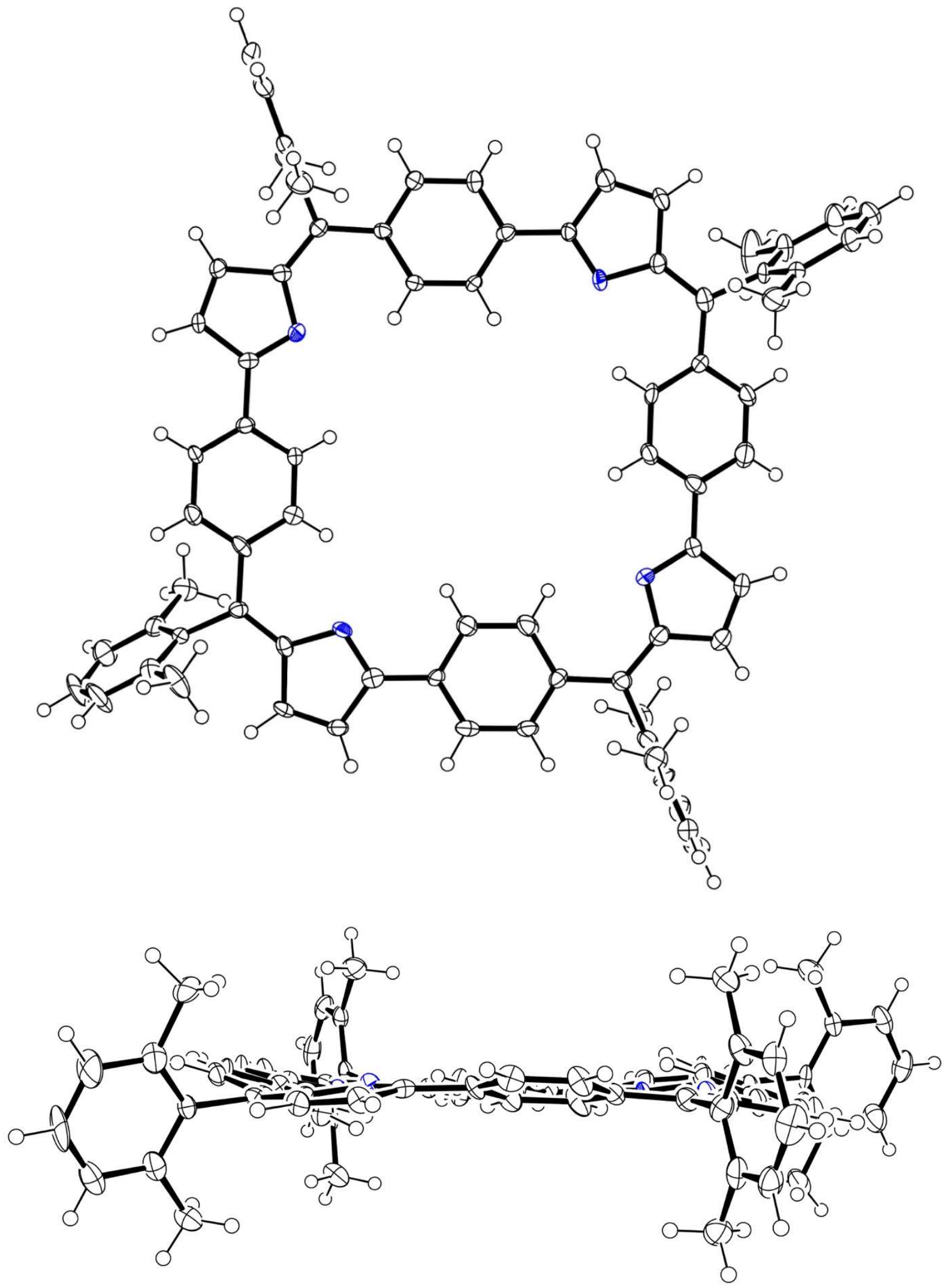

Figure S5-1: ORTEP drawing of 4T. The thermal ellipsoids are scaled to the 50\% probability level. The solvent molecules are omitted for clarity. 


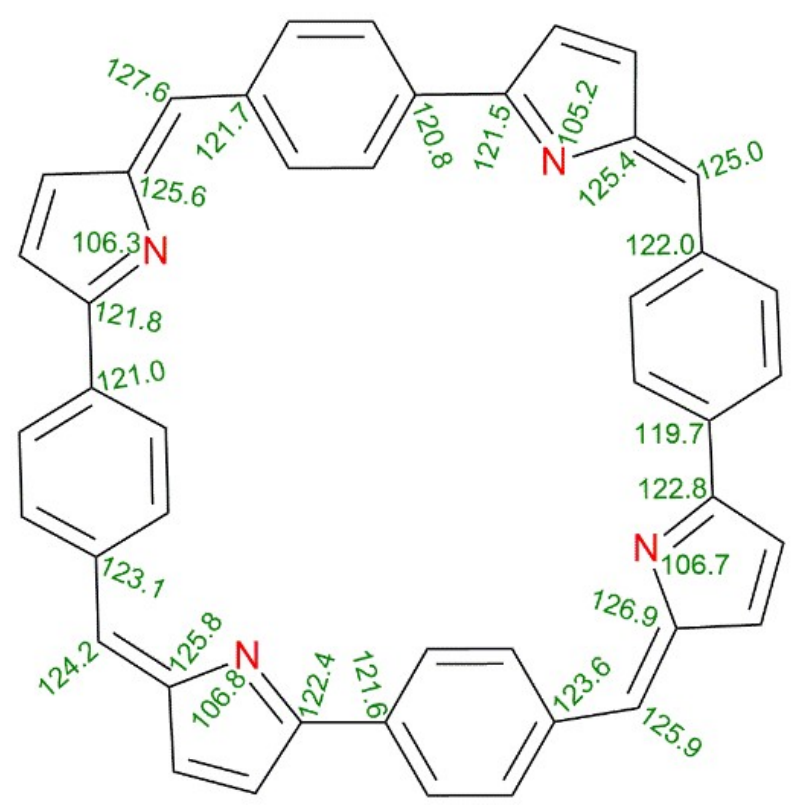

Figure S5-2: Bond angles $\left(^{\circ}\right)$ of 4 T

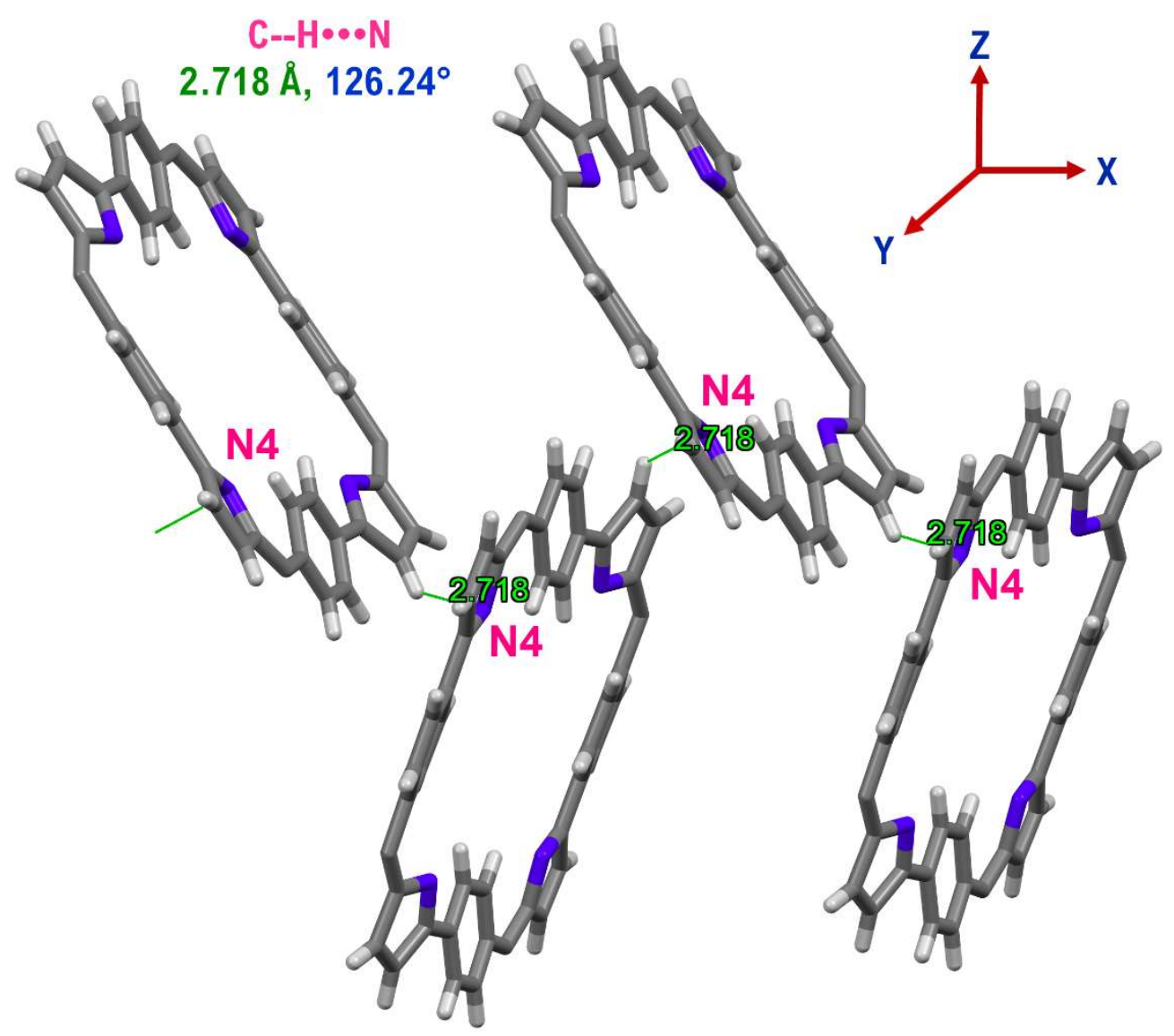

Figure S5-3: Secondary $(\mathrm{C}-\mathrm{H} \bullet \bullet \mathrm{N})$ interactions in 4T. View along $\mathrm{X}$-axis 
Table S5-1: Crystal data and structure refinements of 4T

\begin{tabular}{|c|c|}
\hline Compound & $7 *$ \\
\hline Empirical formula & $\begin{array}{c}\mathrm{C}_{76} \mathrm{H}_{60} \mathrm{~N}_{4}, \mathrm{C}_{7} \mathrm{H}_{5} \mathrm{~N}, \\
0.683\left(\mathrm{C}_{6} \mathrm{H}_{5} \mathrm{NO}_{2}\right)\end{array}$ \\
\hline Formula weight & 1216.52 \\
\hline Temperature/K & 100 \\
\hline Crystal system & orthorhombic \\
\hline Space group & $\operatorname{Pca2}_{1}(\# 29)$ \\
\hline$a / \AA$ & $14.3886(6)$ \\
\hline$b / \AA$ & $19.7475(8)$ \\
\hline$c / \AA$ & $25.8150(11)$ \\
\hline$\alpha /^{\circ}$ & 90 \\
\hline$\beta /^{\circ}$ & 90 \\
\hline$\gamma /{ }^{\circ}$ & 90 \\
\hline Volume $/ \AA^{3}$ & $7335.0(5)$ \\
\hline $\mathrm{Z}$ & 4 \\
\hline$\rho_{\text {calc }} \mathrm{g} / \mathrm{cm}^{3}$ & 1.102 \\
\hline$\mu / \mathrm{mm}^{-1}$ & 0.065 \\
\hline$F(000)$ & 2567.0 \\
\hline Crystal size $/ \mathrm{mm}^{3}$ & $0.1 \times 0.02 \times 0.02$ \\
\hline Radiation & $\operatorname{MoK} \alpha(\lambda=0.71073)$ \\
\hline $2 \Theta$ range for data collection $/{ }^{\circ}$ & 3.156 to 56 \\
\hline Index ranges & $\begin{array}{c}-19 \leq h \leq 19 \\
-26 \leq k \leq 26 \\
-34 \leq l \leq 34\end{array}$ \\
\hline Reflections collected & 121590 \\
\hline Independent reflections & $\begin{array}{c}17687[\text { Rint }=0.1540, \\
R \text { sigma }=0.1194]\end{array}$ \\
\hline Data/restraints/parameters & $7687 / 356 / 883$ \\
\hline Goodness-of-fit $[I>=2 \sigma(I)]$ & 1.020 \\
\hline Final $R$ indexes $[I>=2 \sigma(I)]$ & $\begin{array}{l}R_{1}=0.0922 \\
w R_{2}=0.2181\end{array}$ \\
\hline Final $R$ indexes [all data] & $\begin{array}{l}R_{1}=0.1635 \\
w R_{2}=0.2462\end{array}$ \\
\hline Largest diff. peak/hole / e $\AA^{-3}$ & $0.59 /-0.32$ \\
\hline CCDC Number & 2121585 \\
\hline
\end{tabular}

* A lot of electron peaks are found around the cyclotetramer molecule. Only one nitrobenzene and one benzonitrile molecules can be properly modeled. There are probably more than one each of nitrobenzene and benzonitrile molecules. These electron peaks are removed by the PLAON-SQUEEZE technique and the rest molecules are refined. 


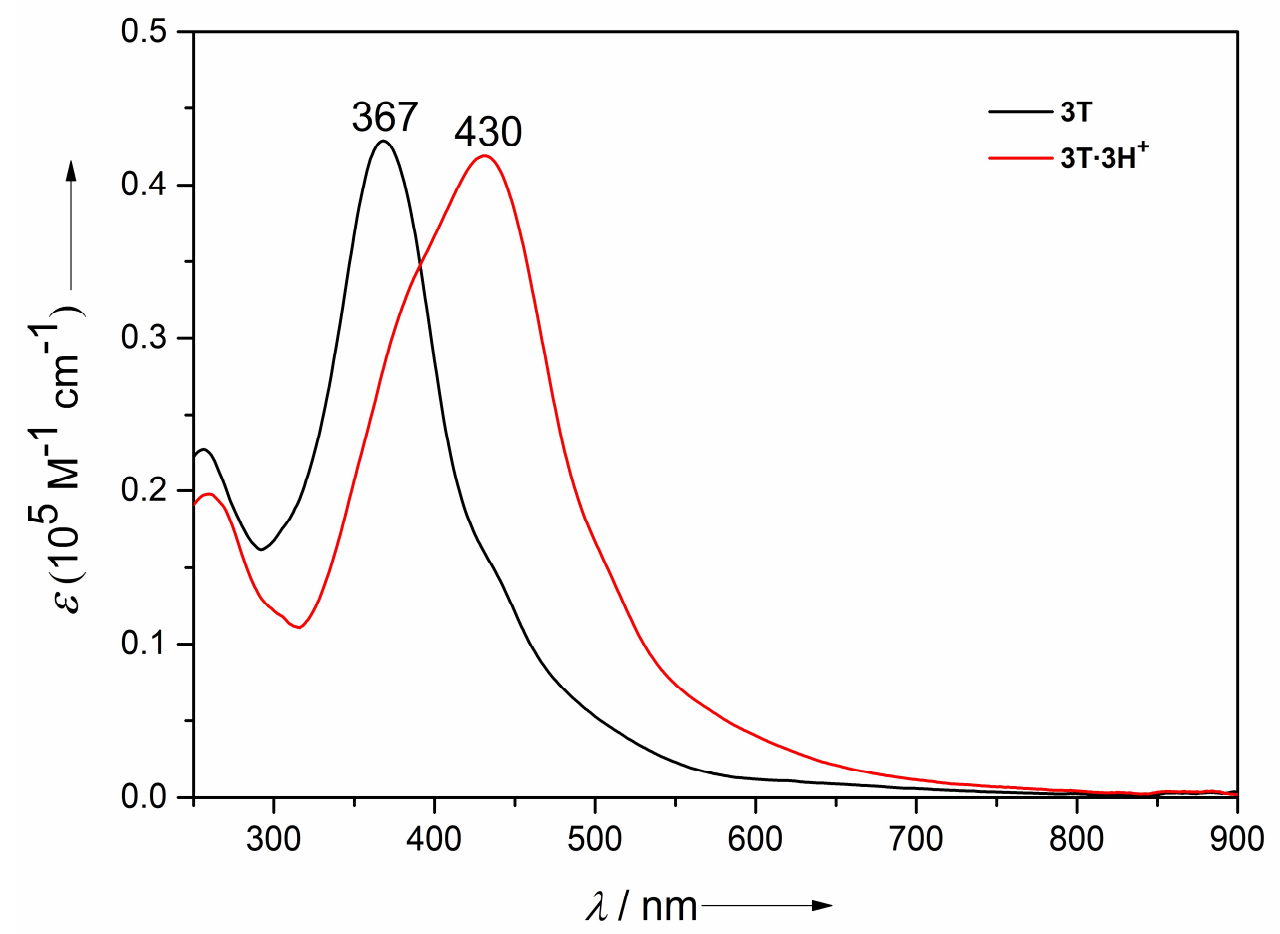

Figure S6-1: Absorption spectra of $\mathbf{3 T}$ with protonated form (dilute $\mathrm{CF}_{3} \mathrm{COOH}$ was used in aliquots) in $\mathrm{CH}_{2} \mathrm{Cl}_{2}$ at $298 \mathrm{~K}$. [3T $]=2.09 \times 10^{-5} \mathrm{M}$; $[\mathrm{TFA}]=4.32 \times 10^{-2} \mathrm{M}$.

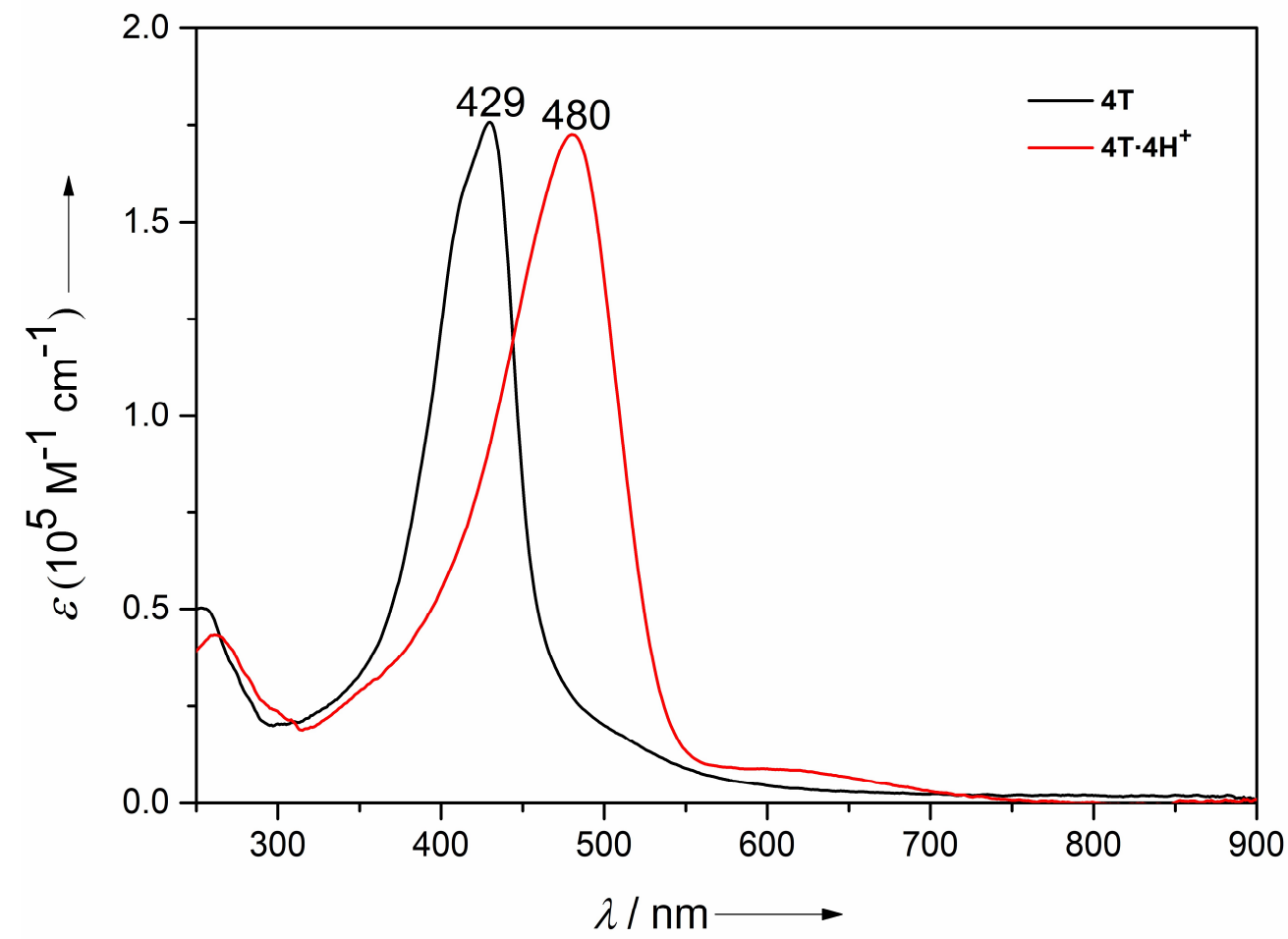

Figure S6-2: Absorption spectra of $\mathbf{4 T}$ along with its protonated form (dilute $\mathrm{CF}_{3} \mathrm{COOH}$ was used in aliquots) in $\mathrm{CH}_{2} \mathrm{Cl}_{2}$ at $298 \mathrm{~K}$. [4T $]=4.47 \times 10^{-6} \mathrm{M}$; $[\mathrm{TFA}]=4.32 \times 10^{-2} \mathrm{M}$. 


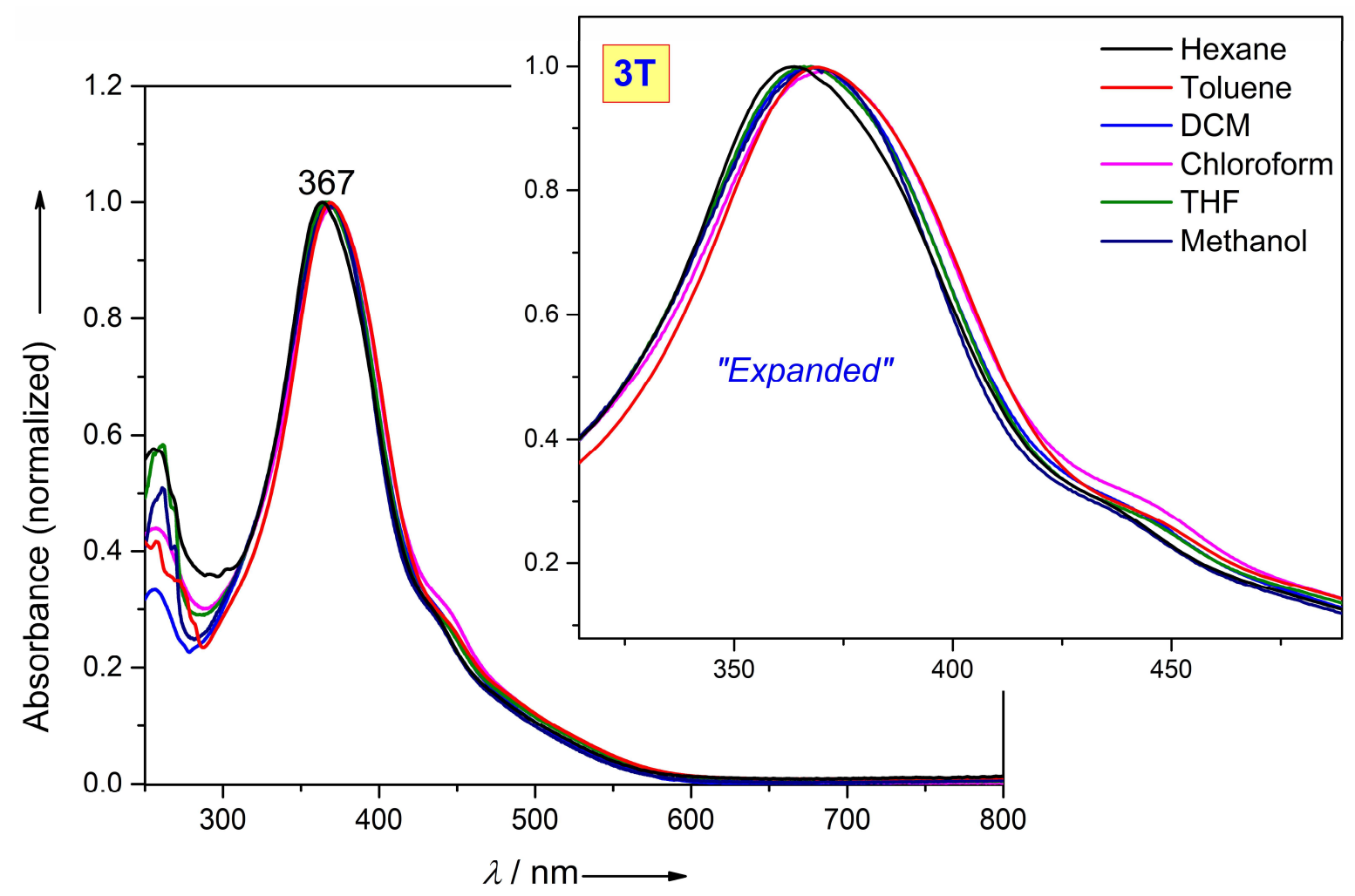

Figure S6-3: Solvent dependant absorption spectra of 3T at $298 \mathrm{~K}$.

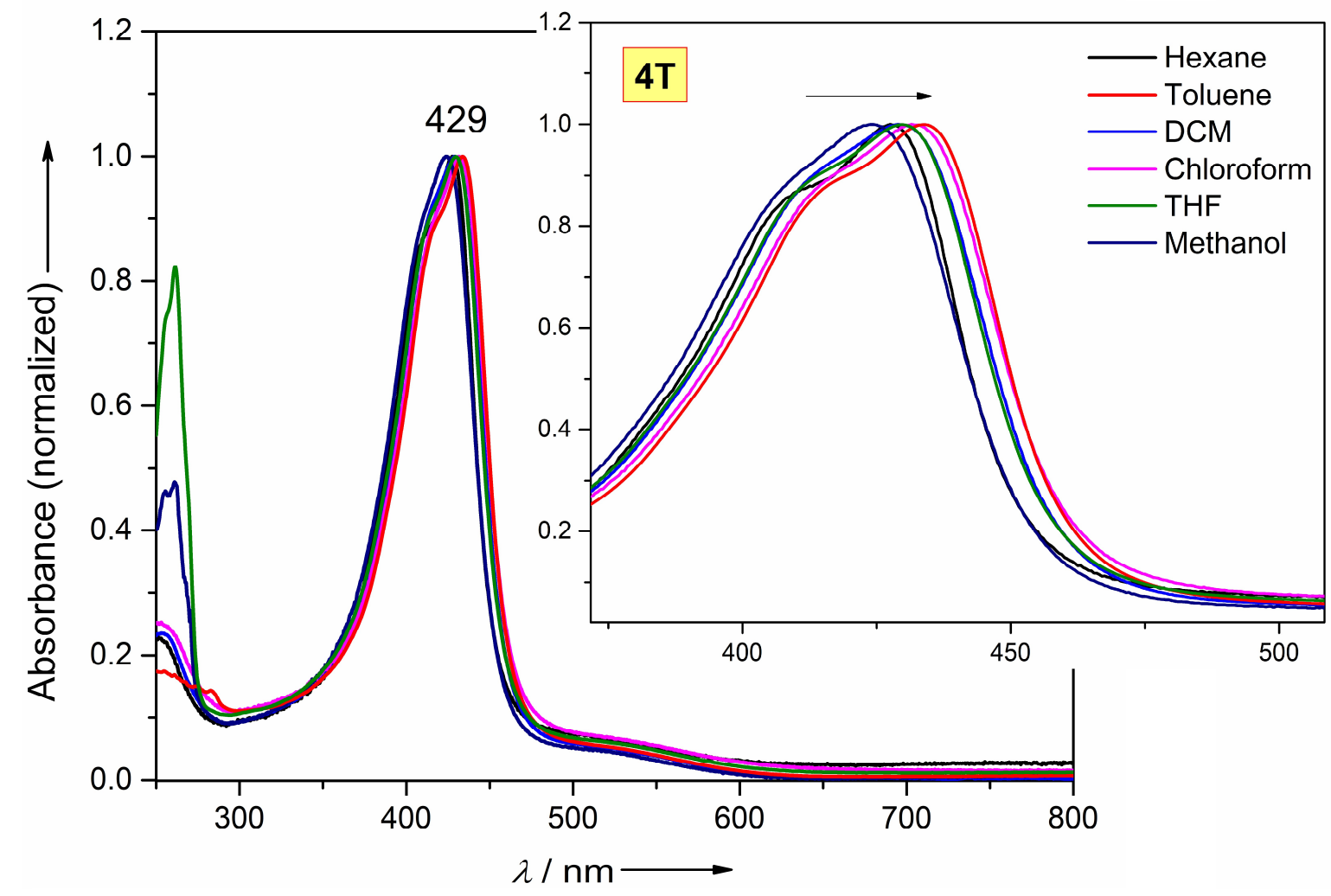

Figure S6-4: Solvent dependant absorption spectra of 4T at $298 \mathrm{~K}$. 


\section{Cyclic Voltammetry}

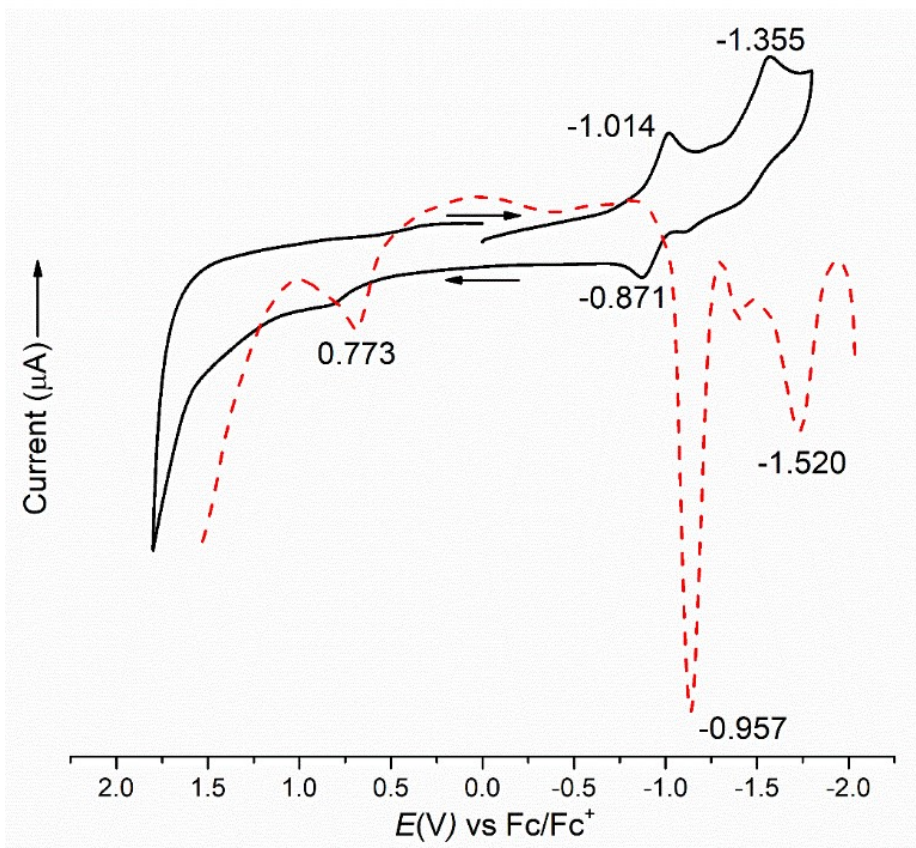

Figure S7-1: Cyclic voltammogram (black line) of 3T and the differential pulse voltammogram (red dotted-line) recorded in $\mathrm{CH}_{2} \mathrm{Cl}_{2}$ with $0.1 \mathrm{M}$ TBAP as the supporting electrolyte at a scan rate of $100 \mathrm{mVs}^{-1}$. Ferrocene/ferrocenium $\left(\mathrm{Fc} / \mathrm{Fc}^{+}\right)$was used as an external reference.

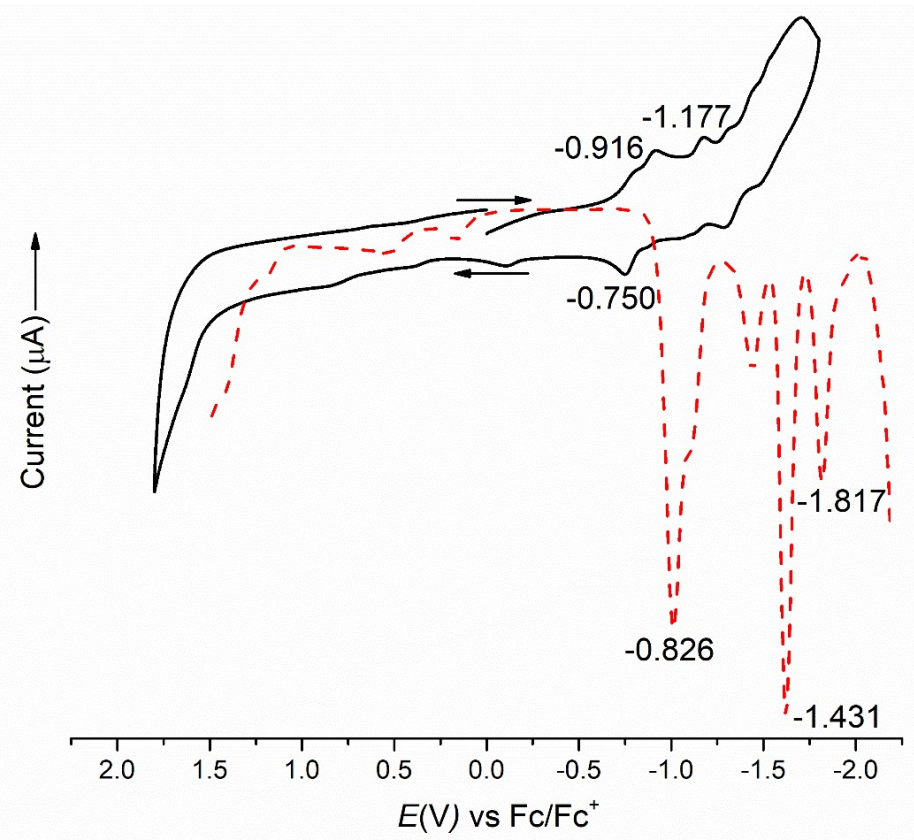

Figure S7-2: Cyclic voltammogram (black line) of $4 \mathrm{~T}$ and the differential pulse voltammogram (red dotted-line) recorded in $\mathrm{CH}_{2} \mathrm{Cl}_{2}$ with $0.1 \mathrm{M}$ TBAP as the supporting electrolyte at a scan rate of $100 \mathrm{mVs}^{-1}$. Ferrocene/ferrocenium $\left(\mathrm{Fc} / \mathrm{Fc}^{+}\right)$was used as an external reference. 


\section{DFT Calculations}

(a) Optimized structures

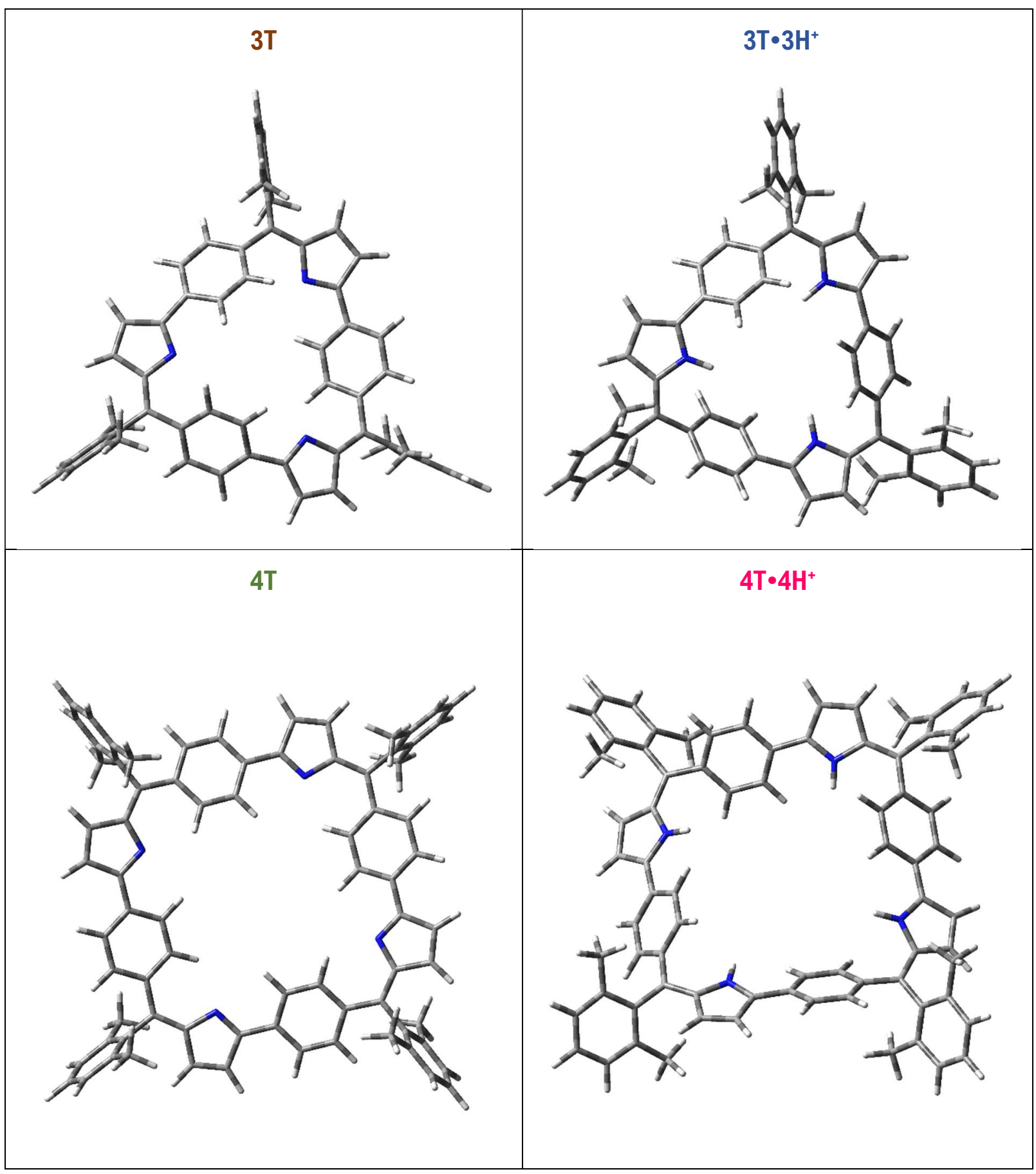

Figure S8-1: DFT Optimized structures for $\mathbf{3 T}, \mathbf{3 T} \bullet \mathbf{3} \mathrm{H}^{+}, \mathbf{4 T}$ and $\mathbf{4 T} \cdot \mathbf{4} \mathrm{H}^{+}$ 


\section{(b) NICS plots}

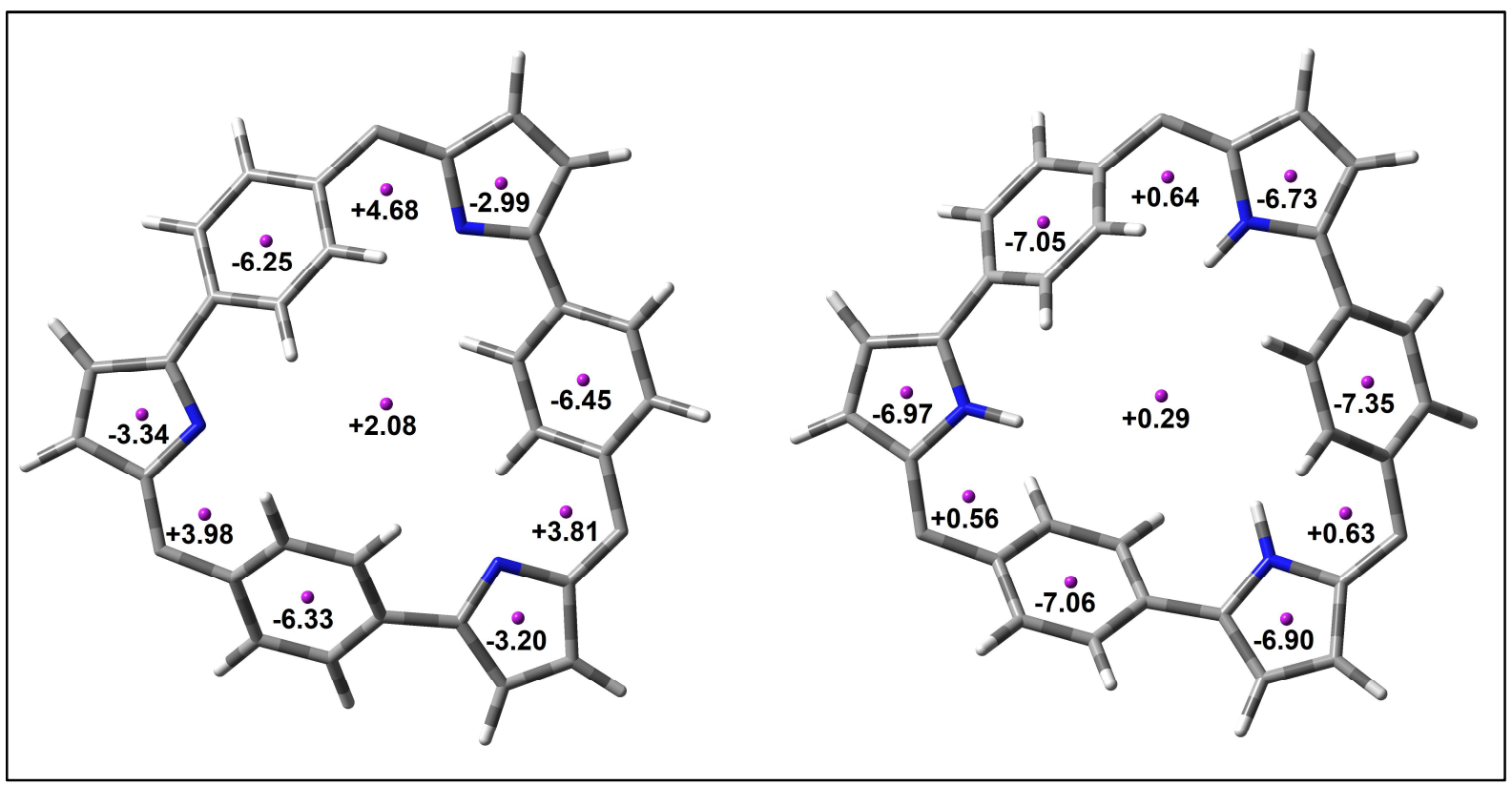

Figure S8-2. NICS $(0)$ values at various positions of $\mathbf{3 T}$ (left) and its protonated form $\mathbf{3 T} \cdot \mathbf{3} \mathbf{H}^{+}$ (right)

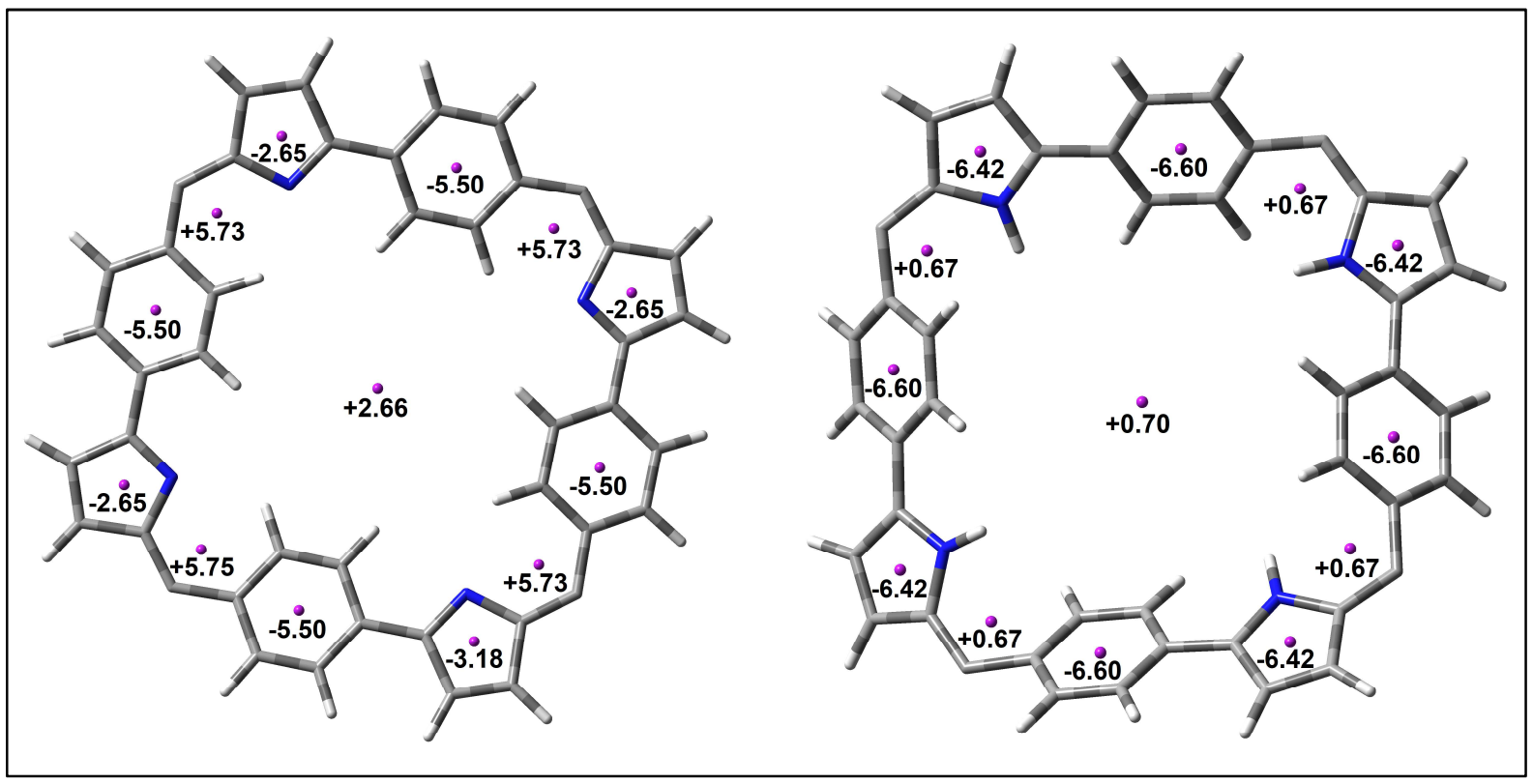

Figure S8-3: $\mathrm{NICS}(0)$ values at various positions of $4 \mathrm{~T}$ (left) and its protonated form $\mathbf{4 T} \cdot \mathbf{4} \mathbf{H}^{+}$(right). 


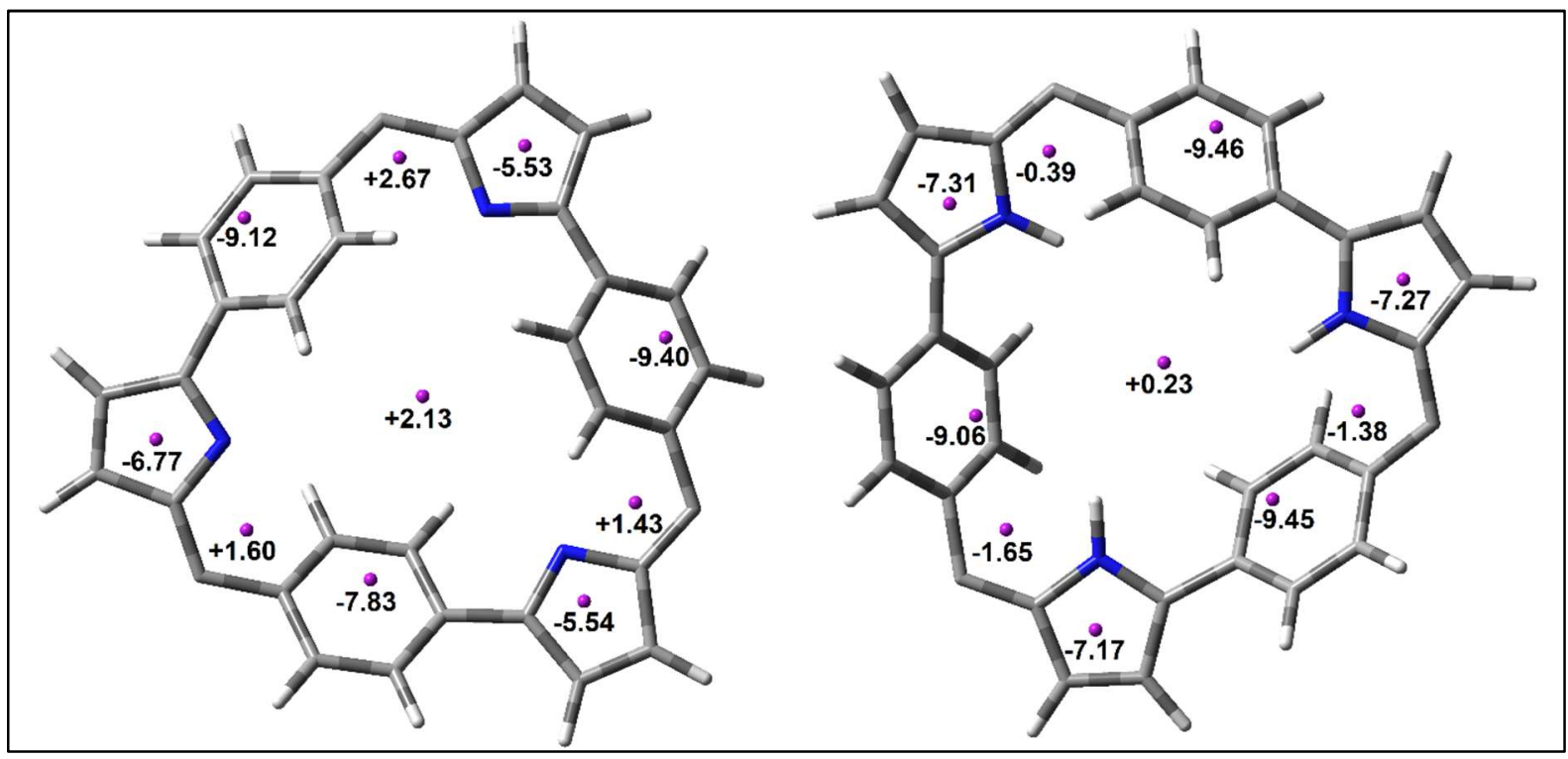

Figure S8-4. NICS(1) $)_{z z}$ indices at various positions of $\mathbf{3 T}(\mathrm{left})$ and its protonated form $\mathbf{3 T} \cdot \mathbf{3} \mathbf{H}^{+}$ (right)

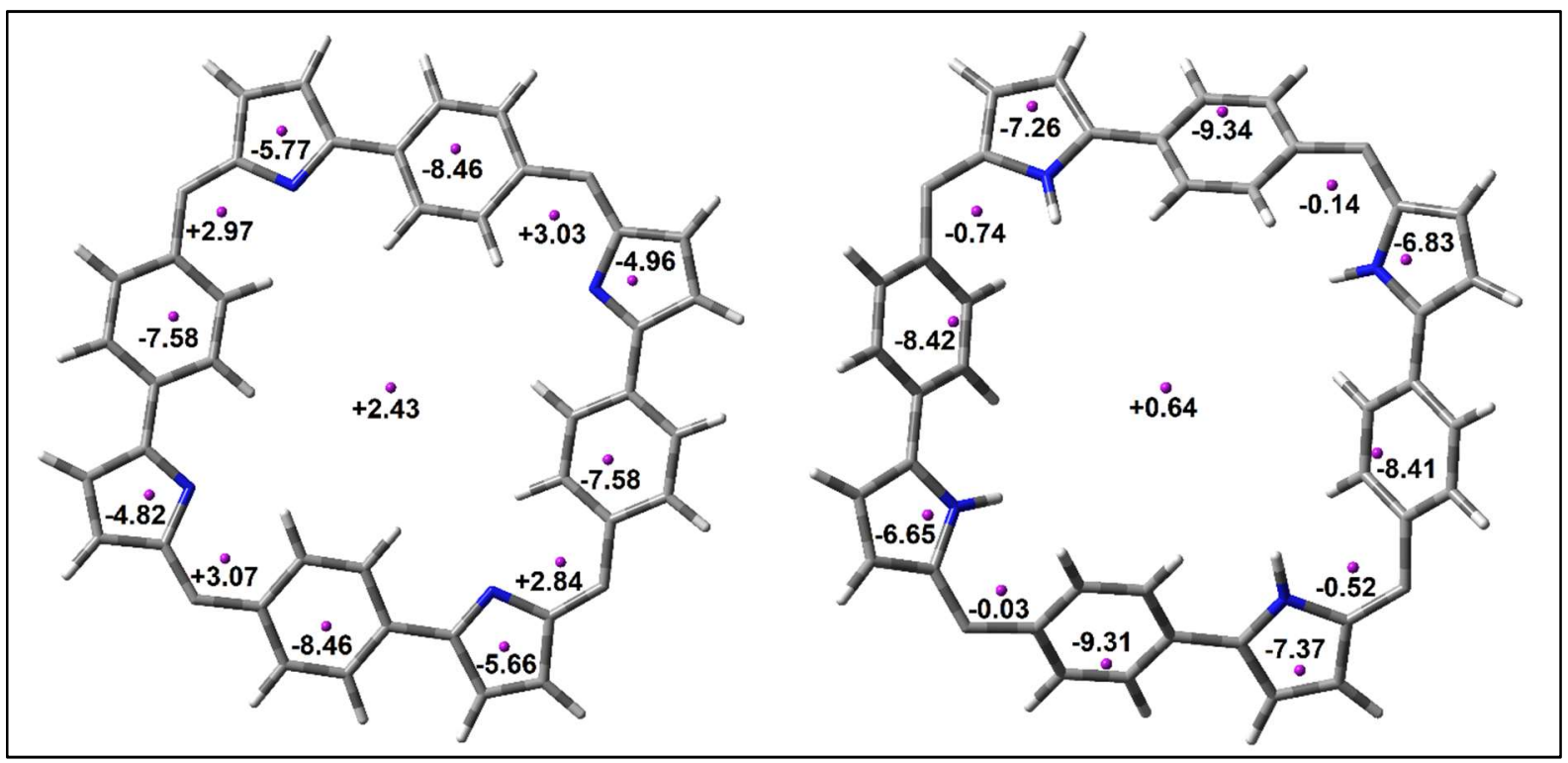

Figure S8-5. NICS(1) $)_{\mathrm{zz}}$ indices at various positions of $4 \mathrm{~T}$ (left) and its protonated form $\mathbf{4 T} \cdot \mathbf{4} \mathbf{H}^{+}$ (right). 
(c) Energy level diagrams and AICD plots
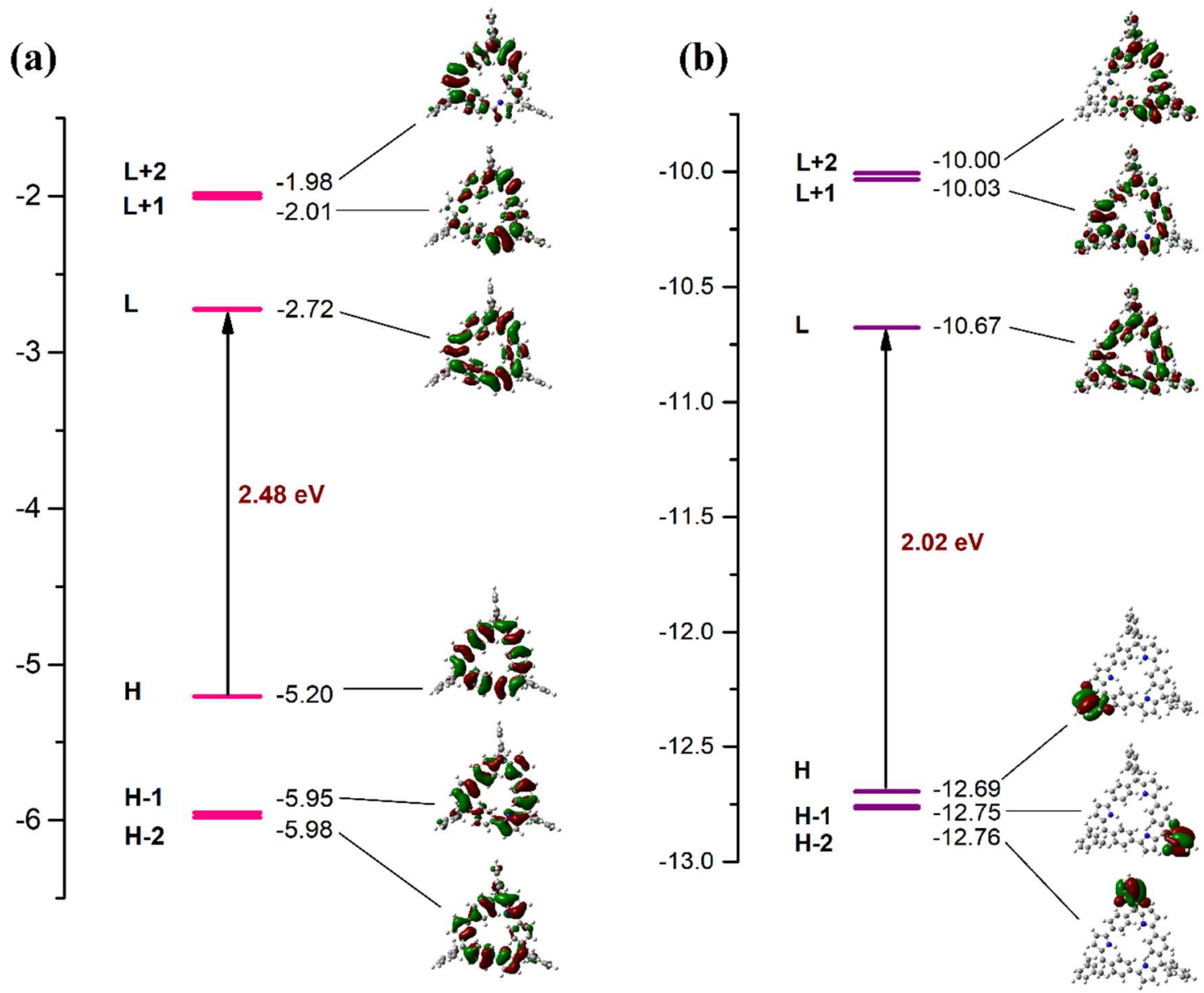

Figure S8-6: Selected Frontier molecular orbital with its energy values in eV of (a) 3T and (b) $\mathbf{3 T} \cdot \mathbf{3} \mathbf{H}^{+}$.
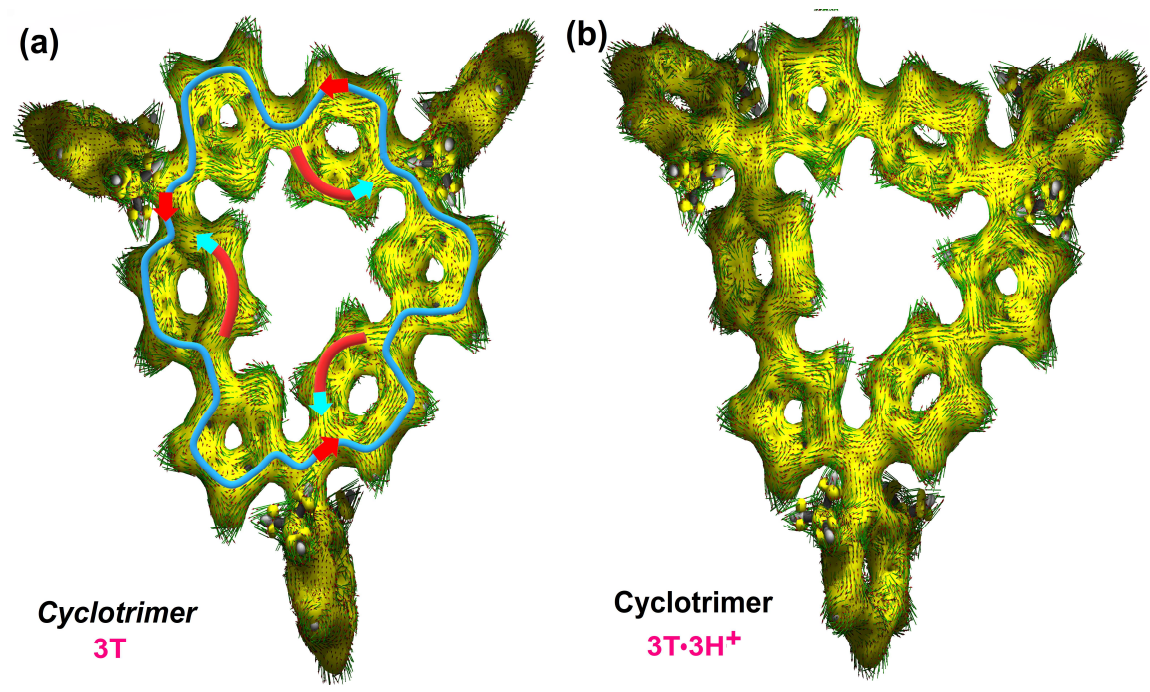

Figure S8-7: ACID plots of (a) 3T (clockwise and counterclockwise vectors are shown in arrows) and (b) $\mathbf{3 T} \cdot \mathbf{3} \mathbf{H}^{+}$. 
(a)

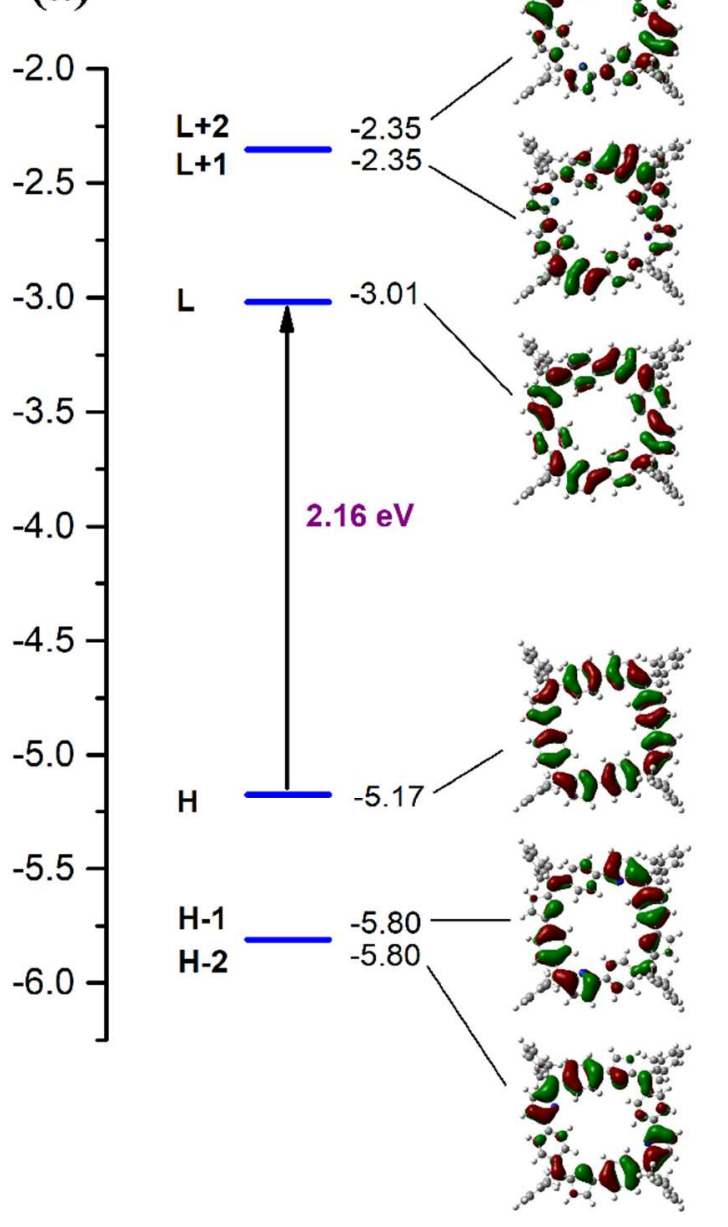

(b)
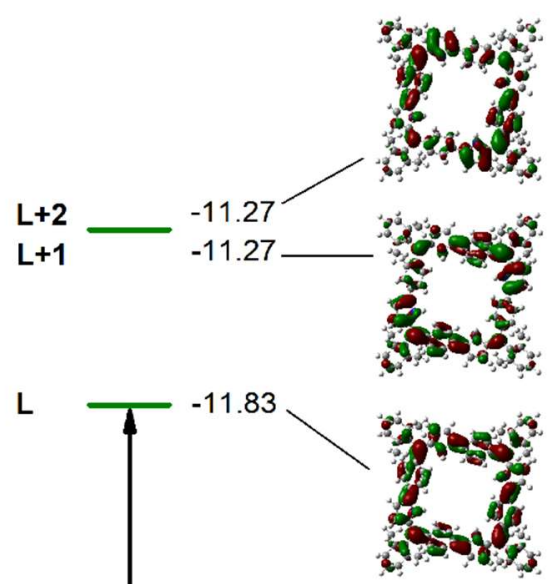

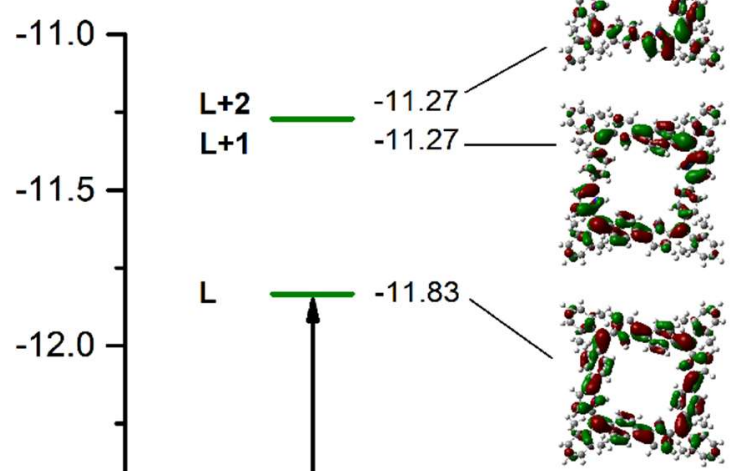

$1.81 \mathrm{eV}$
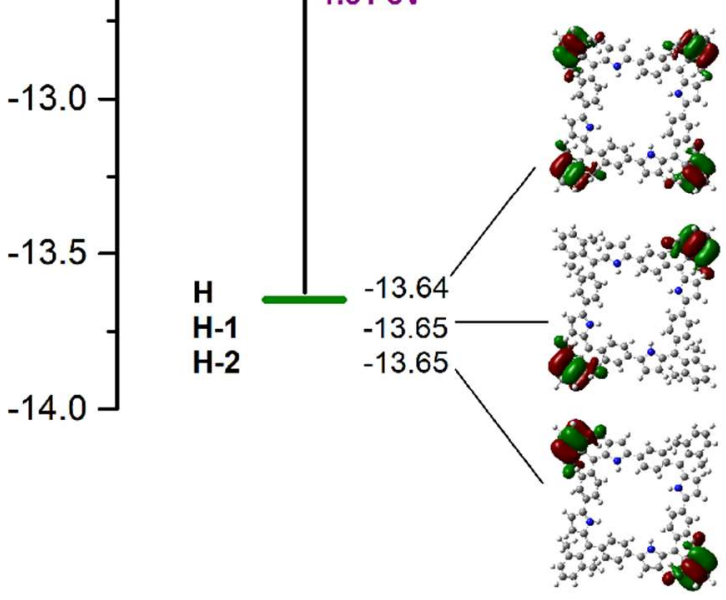

Figure S8-8: Selected Frontier molecular orbital with its energy values in eV of (a) 4T and (b) $\mathbf{4 T} \cdot \mathbf{4} \mathrm{H}^{+}$.
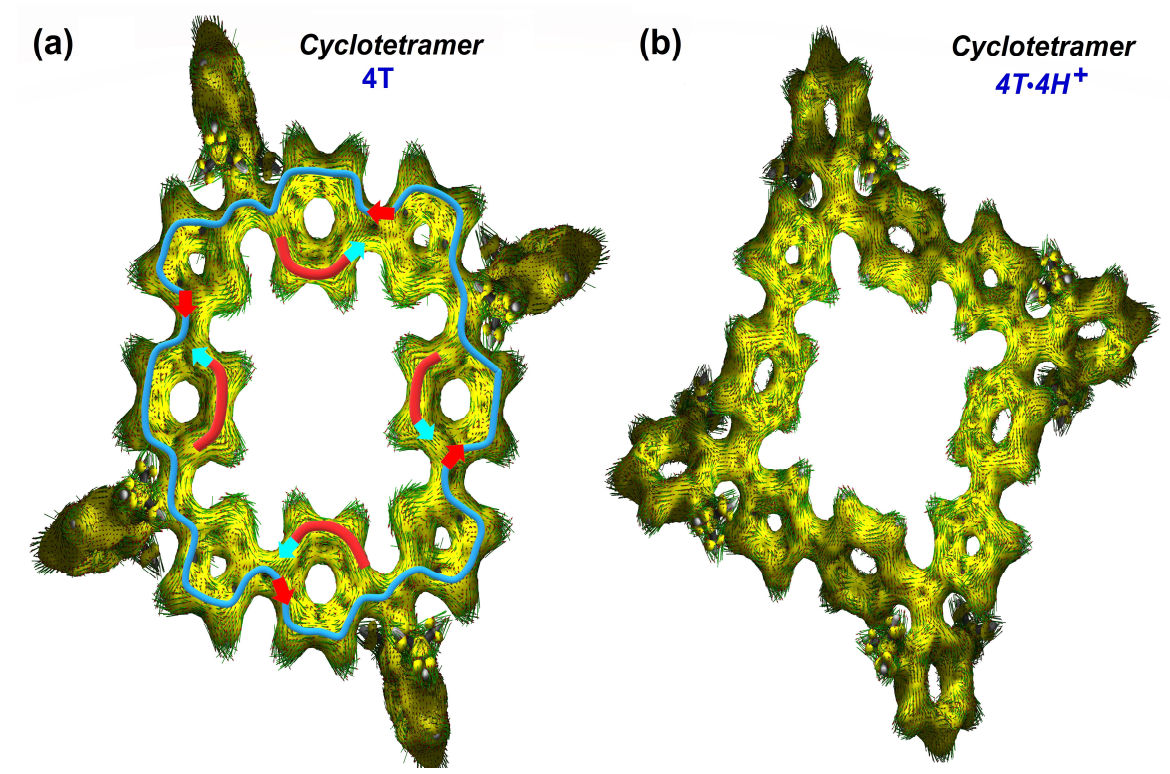

Figure S8-9: ACID plots of (a) 4T (clockwise and counterclockwise vectors are shown in arrows) and (b) $4 \mathbf{T} \bullet 4 \mathbf{H}^{+}$. 


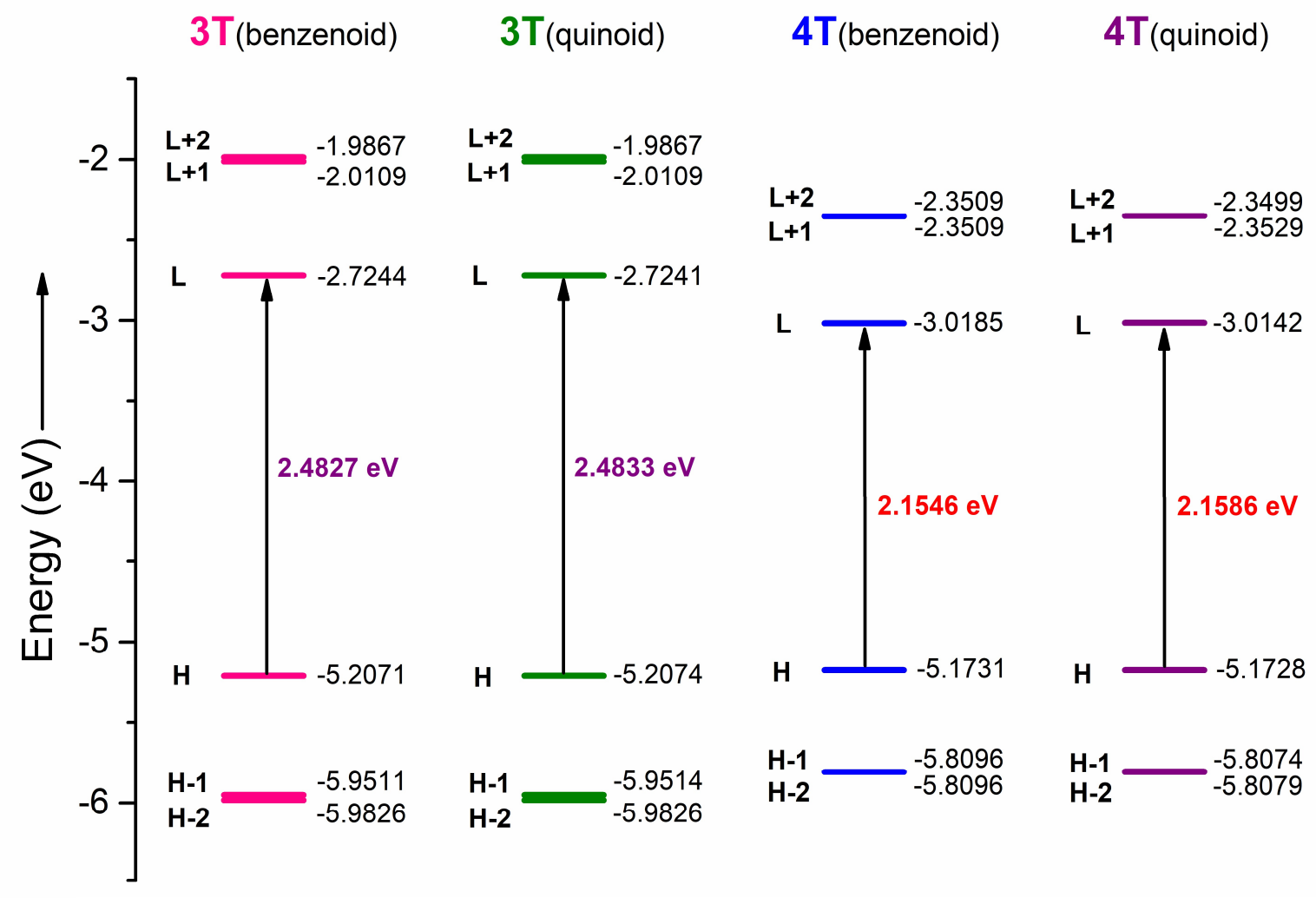

Figure S8-10: Selected potential diagrams of 3T and 4T (both in benzenoid and quinoid form of phenylene linkers) with its energy values in $\mathrm{eV}$.
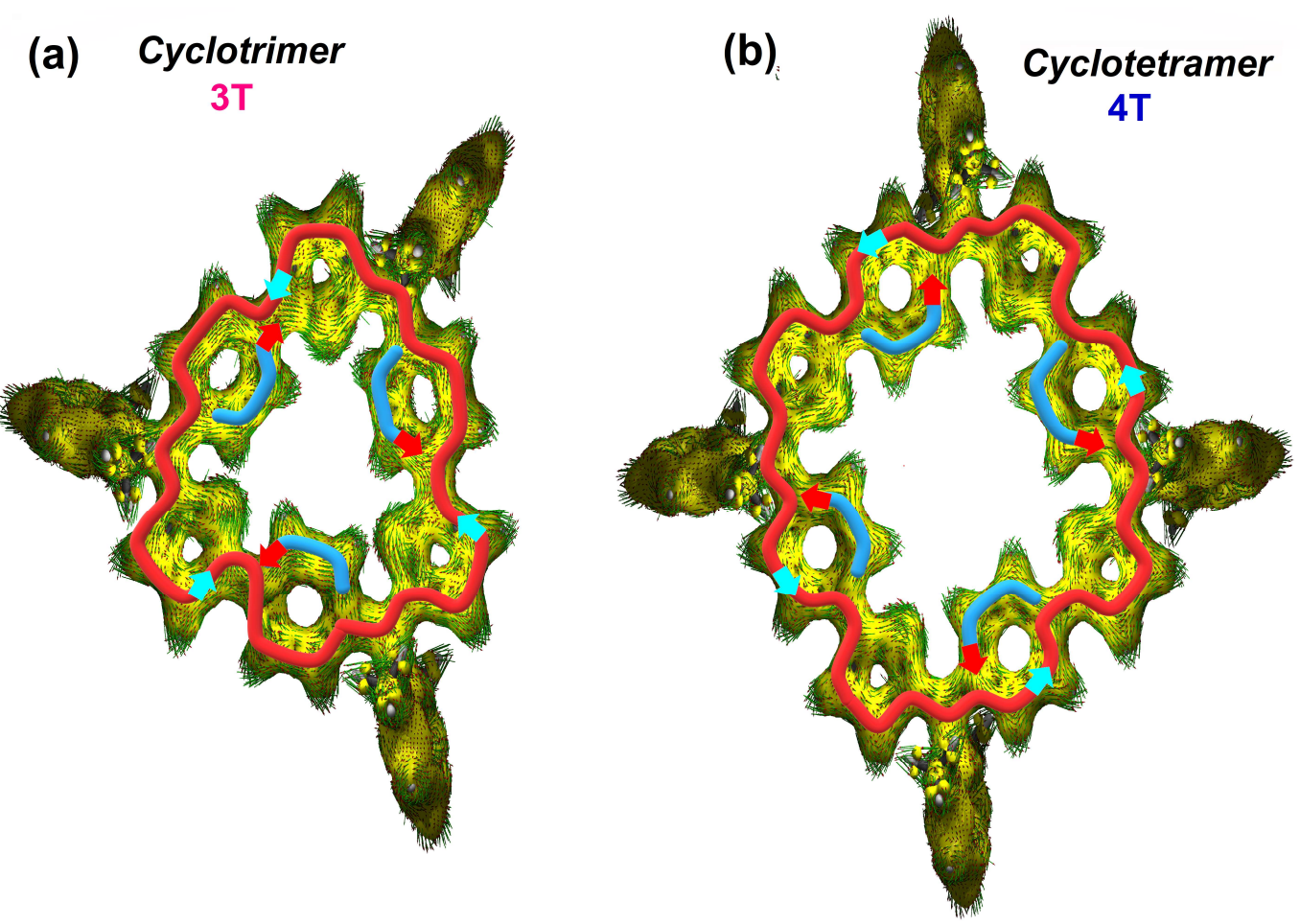

Figure S8-11: ACID plots of (a) 3T-quinoid and (b) 4T-quinoid (clockwise and counterclockwise vectors are shown in arrows). 


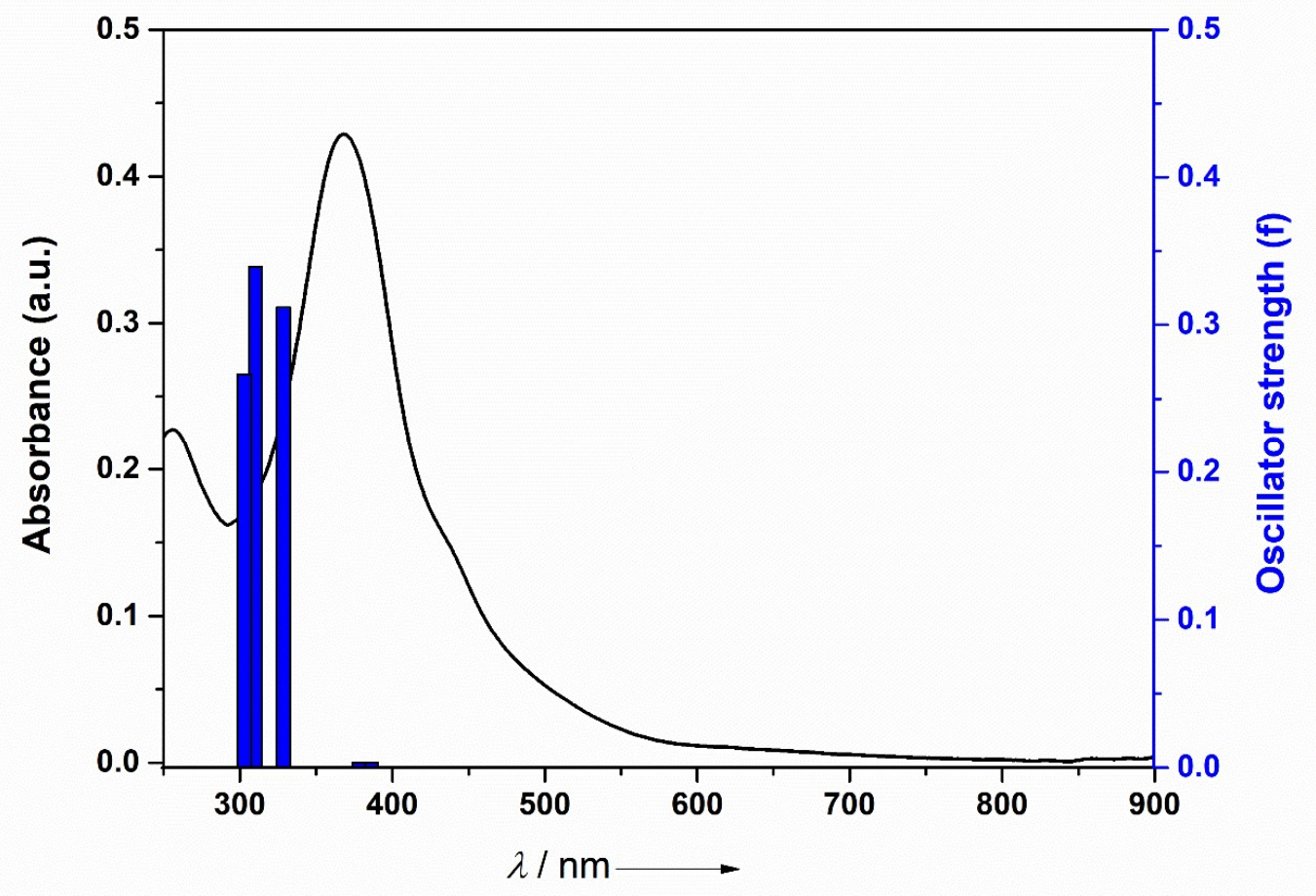

Figure S8-12: Steady-state absorption spectra recorded in $\mathrm{CH}_{2} \mathrm{Cl}_{2}$ along with the theoretical vertical excitation energies obtained from TD-DFT calculations for $\mathbf{3 T}$ carried out at the B3LYP/6-31G(d) level.

Table S8-1: Selected transitions, oscillator strength, symmetry calculated for 3T from DFT analysis at B3LYP/6-31G(d) level of theory $(\mathrm{H}=\mathrm{HOMO}, \mathrm{L}=\mathrm{LUMO}$

\begin{tabular}{|l|l|l|l|l|l|}
\hline No. & $\begin{array}{l}\text { Energy } \\
(\mathrm{cm}-1)\end{array}$ & $\begin{array}{l}\text { Wavelength } \\
(\mathrm{nm})\end{array}$ & $\begin{array}{l}\text { Oscillator } \\
\text { Strength }\end{array}$ & Symmetry & Major contributions \\
\hline 1 & 15558.43 & 642.75 & 0.0002 & Singlet-A & HOMO->LUMO (70\%) \\
\hline 2 & 22288.32 & 448.66 & 0.0034 & Singlet-A & $\begin{array}{l}\text { H-2->LUMO (14\%), H-1->L (51\%), } \\
\text { H->L+1 (44) }\end{array}$ \\
\hline 3 & 22456.08 & 445.31 & 0.0035 & Singlet-A & $\begin{array}{l}\text { H-2->L(47\%), H->L+1(20\%),H- } \\
>\text { L+2(43\%) }\end{array}$ \\
\hline 4 & 23563.48 & 424.38 & 0.3119 & Singlet-A & $\begin{array}{l}\text { H-6->L(10\%), H-1->L(26\%), H- } \\
>\text { L+2(39\%) }\end{array}$ \\
\hline 5 & 24004.67 & 416.58 & 0.3393 & Singlet-A & H-13->L(11\%), H->L+1(33\%) \\
\hline 6 & 24178.88 & 413.58 & 0.2667 & Singlet-A & $\begin{array}{l}\text { H-6->L(14\%), H-3->L(39\%), H- } \\
>\text { L+1(23\%), H->L+2(28\%) }\end{array}$ \\
\hline
\end{tabular}




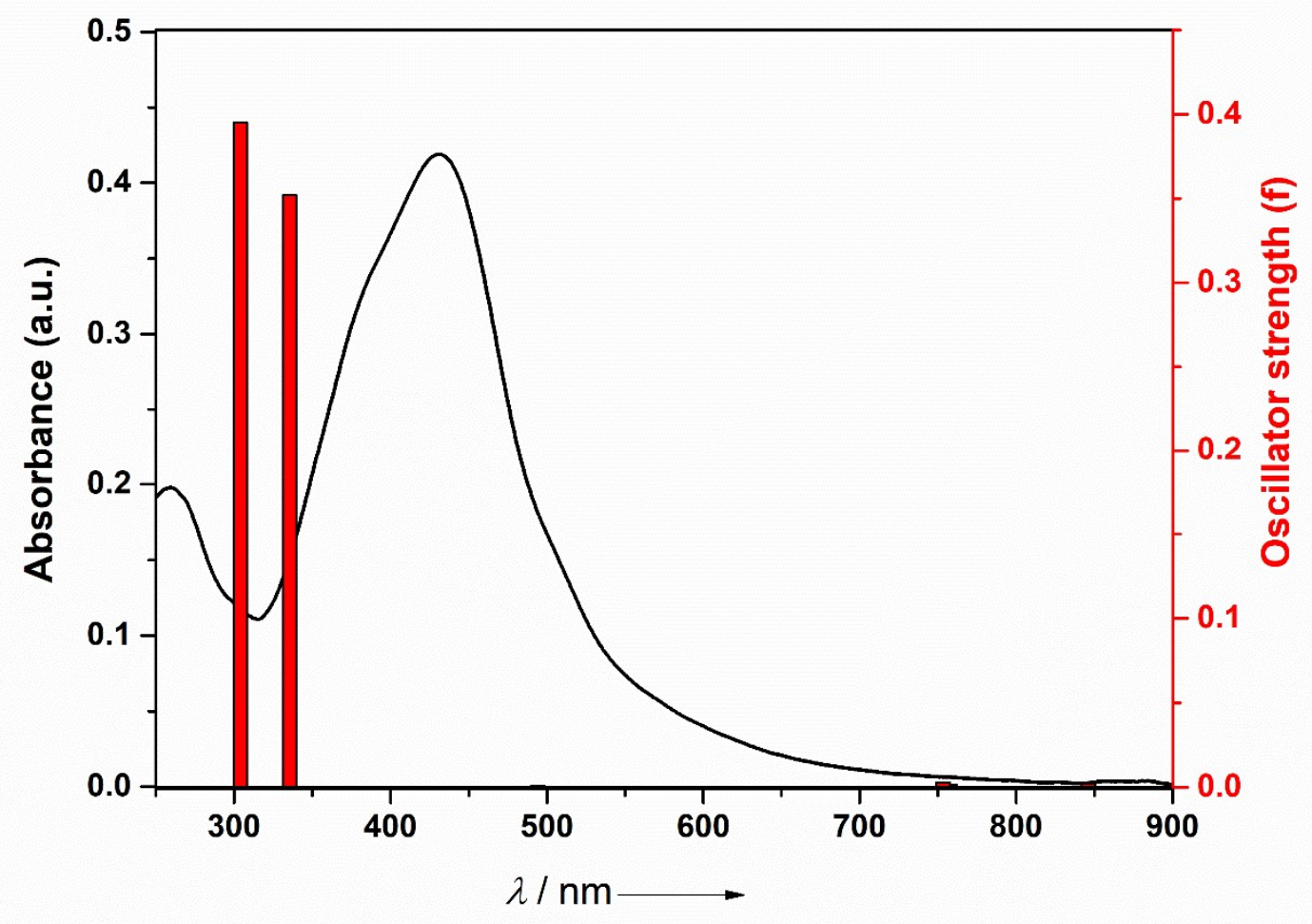

Figure S8-13: Steady-state absorption spectra recorded in $\mathrm{CH}_{2} \mathrm{Cl}_{2}$ along with the theoretical vertical excitation energies obtained from TD-DFT calculations for $\mathbf{3 T} \cdot \mathbf{3} \mathbf{H}^{+}$carried out at the B3LYP/6-31G(d) level.

Table S8-2: Selected transitions, oscillator strength, symmetry calculated for $\mathbf{3 T \cdot} \cdot \mathbf{3} \mathbf{H}^{+}$from DFT analysis at B3LYP/6-31G(d) level of theory $(\mathrm{H}=\mathrm{HOMO}, \mathrm{L}=\mathrm{LUMO}$

\begin{tabular}{|l|l|l|l|l|l|}
\hline No. & $\begin{array}{l}\text { Energy } \\
(\mathrm{cm}-1)\end{array}$ & $\begin{array}{l}\text { Wavelength } \\
(\mathrm{nm})\end{array}$ & $\begin{array}{l}\text { Oscillator } \\
\text { Strength }\end{array}$ & Symmetry & Major contributions \\
\hline 1 & 12444.32 & 803.58 & 0.0019 & Singlet-A & HOMO->LUMO (67\%), H->L+1(22\%) \\
\hline 2 & 12982.30 & 770.29 & 0.0016 & Singlet-A & H-2->LUMO (65\%), H-1->L(17\%) \\
\hline 3 & 13011.33 & 768.57 & 0.0029 & Singlet-A & H-1->L(65\%), H-1->L+2(19\%) \\
\hline 4 & 14907.54 & 670.81 & 0.0007 & Singlet-A & H-6->L(15\%), H-3->L(68\%) \\
\hline 5 & 16365.79 & 611.02 & 0.3519 & Singlet-A & H-4->L(69\%) \\
\hline 6 & 16687.67 & 599.23 & 0.3948 & Singlet-A & H-5->L(70\%) \\
\hline
\end{tabular}




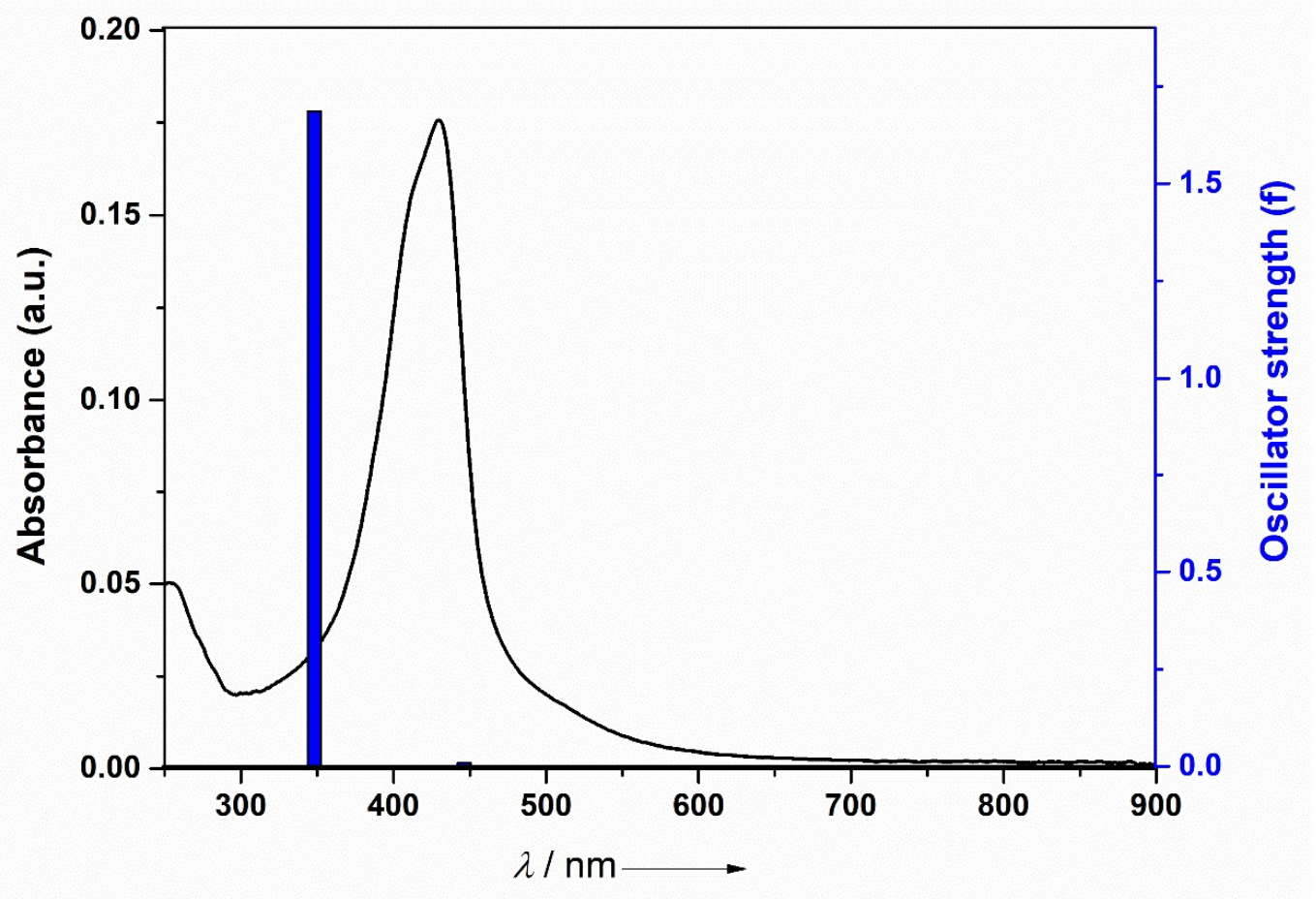

Figure S8-14: Steady-state absorption spectra recorded in $\mathrm{CH}_{2} \mathrm{Cl}_{2}$ along with the theoretical vertical excitation energies obtained from TD-DFT calculations for $4 \mathrm{~T}$ carried out at the B3LYP/6-31G(d) level.

Table S8-3: Selected transitions, oscillator strength, symmetry calculated for 4T from DFT analysis at B3LYP/6-31G(d) level of theory $(\mathrm{H}=\mathrm{HOMO}, \mathrm{L}=\mathrm{LUMO}$

\begin{tabular}{|l|l|l|l|l|l|}
\hline No. & $\begin{array}{l}\text { Energy } \\
(\mathrm{cm}-1)\end{array}$ & $\begin{array}{l}\text { Wavelength } \\
(\mathrm{nm})\end{array}$ & $\begin{array}{l}\text { Oscillator } \\
\text { Strength }\end{array}$ & Symmetry & Major contributions \\
\hline 1 & 13549.31 & 738.04 & 0 & Singlet-A & H->L(70\%) \\
\hline 2 & 19688.80 & 507.90 & 0.0085 & Singlet-B & H-1->L(53\%), H->L+2(12\%) \\
\hline 3 & 19688.80 & 507.90 & 0.0085 & Singlet-B & H-2->L $(53 \%)$ \\
\hline 4 & 22161.70 & 451.22 & 1.6852 & Singlet-B & $\begin{array}{l}\text { H-2->L }(19 \%), \mathrm{H}-1->\mathrm{L}(40 \%), \mathrm{H}- \\
>\mathrm{L}+1(52 \%)\end{array}$ \\
\hline 5 & 22161.70 & 451.22 & 1.6852 & Singlet-B & H-2->L(40\%), H->L+2(52\%) \\
\hline 6 & 23511.87 & 425.32 & 0.0002 & Singlet-A & H-3->L $(68 \%)$ \\
\hline
\end{tabular}




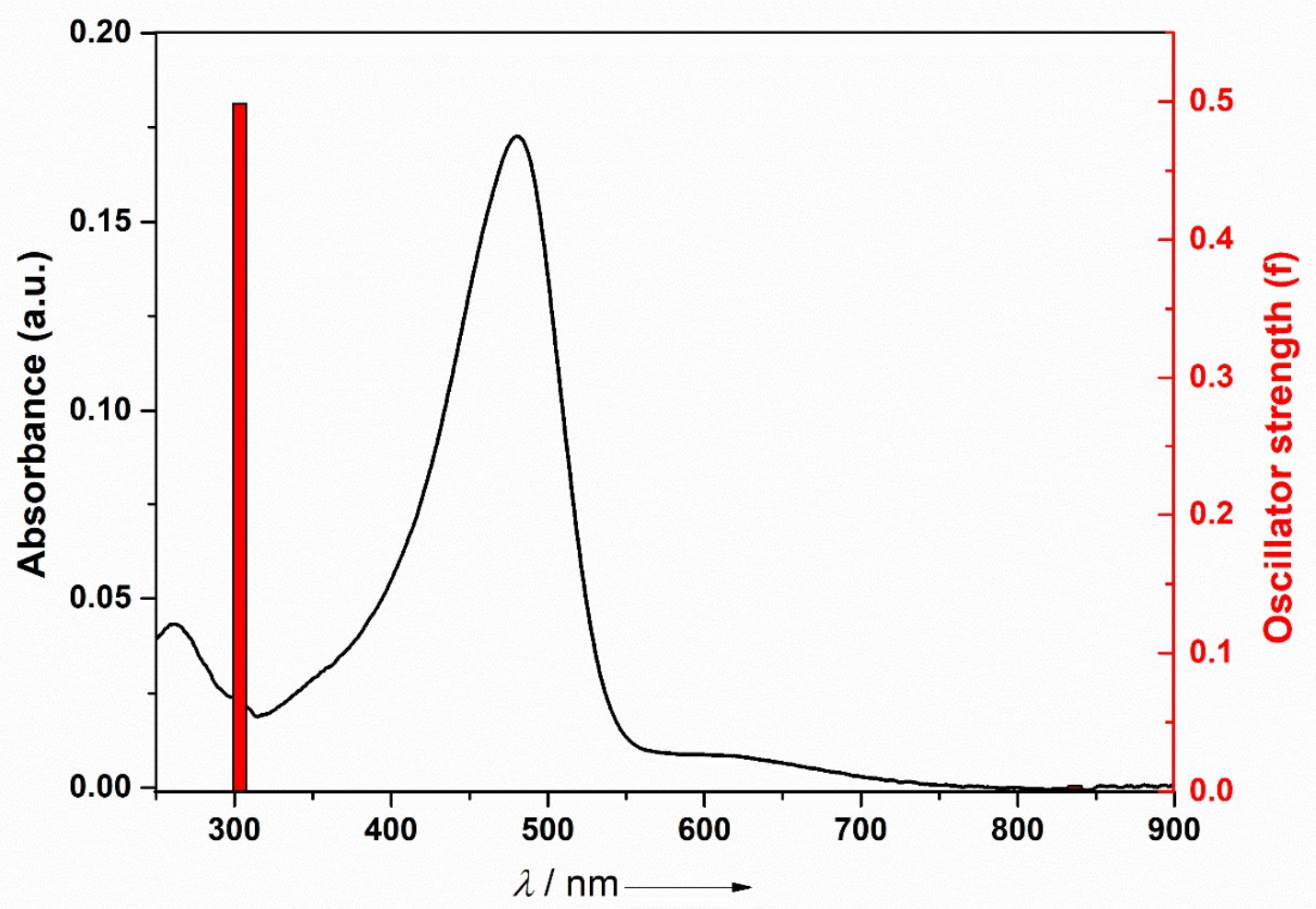

Figure S8-15: Steady-state absorption spectra recorded in $\mathrm{CH}_{2} \mathrm{Cl}_{2}$ along with the theoretical

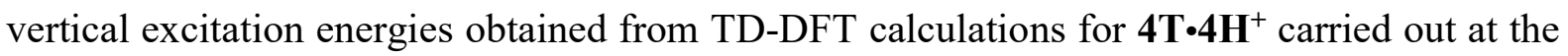
B3LYP/6-31G(d) level.

Table S8-4: Selected transitions, oscillator strength, symmetry calculated for $\mathbf{4 T} \cdot \mathbf{4} \mathbf{H}^{+}$from DFT analysis at B3LYP/6-31G(d) level of theory $(\mathrm{H}=\mathrm{HOMO}, \mathrm{L}=\mathrm{LUMO}$

\begin{tabular}{|l|l|l|l|l|l|}
\hline No. & $\begin{array}{l}\text { Energy } \\
(\mathrm{cm}-1)\end{array}$ & $\begin{array}{l}\text { Wavelength } \\
(\mathrm{nm})\end{array}$ & $\begin{array}{l}\text { Oscillator } \\
\text { Strength }\end{array}$ & Symmetry & Major contributions \\
\hline 1 & 23511.87 & 894.05 & 0 & Singlet-A & H->L(67\%), H-1->L+1(14\%) \\
\hline 2 & 11230.46 & 890.46 & 0.0039 & Singlet-B & $\begin{array}{l}\text { H-3->L+1(14\%), H-1->L(67\%), H- } \\
>\text { L+1(14\%) }\end{array}$ \\
\hline 3 & 11230.46 & 890.45 & 0.0039 & Singlet-B & H-3->L+2(14\%), H-2->L(67\%) \\
\hline 4 & 11233.69 & 890.17 & 0.0018 & Singlet-A & $\begin{array}{l}\text { H-3->L(67\%), H-2->L+2(14\%),H-1- } \\
>\text { L+1(14\%) }\end{array}$ \\
\hline 5 & 13156.52 & 760.08 & 0 & Singlet-A & H-8->L(13\%), H-4->L(69\%) \\
\hline 6 & 14809.95 & 675.21 & 0.4984 & Singlet-B & H-6->L(49\%), H-5->L(50\%) \\
\hline
\end{tabular}


(f) Cartesian coordinates for $3 \mathrm{~T}, 3 \mathrm{~T} \cdot 3 \mathrm{H}^{+}$, $4 \mathrm{~T}$ and $4 \mathrm{~T} \cdot 4 \mathrm{H}^{+}$used in this work.

3T: B3LYP/6-31G(d) level (found 0 imaginary frequencies)

Optimization energy Eopt $=-2363.3152$ Hartree

$$
01
$$

\begin{tabular}{|c|c|c|c|}
\hline Atom & $\mathbf{X}$ & $\mathbf{Y}$ & $\mathbf{Z}$ \\
\hline C & 0.56059600 & 2.52758700 & 0.77692600 \\
\hline C & -0.79667500 & 2.77765800 & 0.76072800 \\
\hline C & -1.31541900 & 3.94972300 & 0.16572000 \\
\hline C & -0.39073900 & 4.90852900 & -0.29968600 \\
\hline C & 0.98196800 & 4.65569100 & -0.27951500 \\
\hline C & 1.47752800 & 3.43800200 & 0.21793900 \\
\hline $\mathrm{H}$ & 0.94129600 & 1.60330500 & 1.19516000 \\
\hline $\mathrm{H}$ & -1.47586000 & 2.05638000 & 1.19185200 \\
\hline H & -0.75589100 & 5.83833600 & -0.72652200 \\
\hline $\mathrm{H}$ & 1.66448300 & 5.38926400 & -0.70113400 \\
\hline C & 3.53359800 & -3.15458400 & 0.38575600 \\
\hline C & 2.29822100 & -3.00579600 & -0.26847200 \\
\hline C & 2.07823700 & -1.82837800 & -1.00703300 \\
\hline C & 2.99973300 & -0.80080700 & -1.00668200 \\
\hline C & 4.18988700 & -0.89040800 & -0.25180400 \\
\hline C & 4.46684200 & -2.11582000 & 0.38793700 \\
\hline $\mathrm{H}$ & 3.74652900 & -4.06203200 & 0.94494500 \\
\hline $\mathrm{H}$ & 1.14712300 & -1.72412800 & -1.55074600 \\
\hline $\mathrm{H}$ & 2.79784200 & 0.09701800 & -1.57397300 \\
\hline $\mathrm{H}$ & 5.39440400 & -2.23144100 & 0.94168300 \\
\hline C & -2.92146100 & -3.14738200 & -0.46327900 \\
\hline C & -4.11982100 & -2.78407500 & 0.18824400 \\
\hline C & -4.59580900 & -1.47314700 & 0.14525300 \\
\hline C & -3.88792500 & -0.48103500 & -0.55226000 \\
\hline C & -2.77823200 & -0.87674800 & -1.32292100 \\
\hline C & -2.31518500 & -2.17876400 & -1.29476300 \\
\hline
\end{tabular}




\begin{tabular}{|c|c|c|c|}
\hline $\mathrm{H}$ & -4.64191500 & -3.51911500 & 0.79331600 \\
\hline $\mathrm{H}$ & -5.47598700 & -1.20643600 & 0.72466400 \\
\hline $\mathrm{H}$ & -2.25702900 & -0.12459600 & -1.90459000 \\
\hline $\mathrm{H}$ & -1.44627100 & -2.45105200 & -1.87579400 \\
\hline C & 4.50050300 & 1.54466400 & -0.00155800 \\
\hline C & 2.87771100 & 2.99837200 & 0.11037300 \\
\hline C & 4.11674500 & 3.77292600 & -0.04133000 \\
\hline C & 5.13087500 & 2.86403400 & -0.07764500 \\
\hline C & -4.14395700 & 0.95967800 & -0.39193400 \\
\hline C & -5.39704100 & 1.67729900 & -0.13102700 \\
\hline C & -5.06474900 & 2.99425500 & -0.03050900 \\
\hline C & -3.60821500 & 3.05980300 & -0.16600700 \\
\hline C & -0.11791600 & -5.77937700 & 0.16559400 \\
\hline C & -0.90815400 & -4.58553300 & -0.14852100 \\
\hline C & 1.18057500 & -3.95676300 & -0.16319500 \\
\hline C & 1.18525500 & -5.38730700 & 0.16687900 \\
\hline C & 5.06422000 & 0.29081200 & -0.10205800 \\
\hline C & -2.77259700 & 4.13845200 & 0.03178600 \\
\hline C & -2.27957600 & -4.44699600 & -0.17904200 \\
\hline $\mathrm{N}$ & 3.12097400 & 1.70756300 & 0.13831600 \\
\hline $\mathrm{N}$ & -3.11489700 & 1.77744900 & -0.42612500 \\
\hline $\mathrm{N}$ & -0.04142200 & -3.50763800 & -0.34600100 \\
\hline $\mathrm{H}$ & 4.19999200 & 4.85230000 & -0.08677500 \\
\hline $\mathrm{H}$ & 6.19046700 & 3.05544800 & -0.19010500 \\
\hline $\mathrm{H}$ & -0.51305000 & -6.77040100 & 0.34794900 \\
\hline $\mathrm{H}$ & 2.05755000 & -6.00058600 & 0.35832800 \\
\hline $\mathrm{H}$ & -5.72071400 & 3.83192100 & 0.16902400 \\
\hline $\mathrm{H}$ & -6.38573900 & 1.23927100 & -0.06305000 \\
\hline C & -3.33646300 & 5.52237300 & 0.10889400 \\
\hline C & -3.37101200 & 6.19798700 & 1.35097400 \\
\hline C & -3.81395200 & 6.15234900 & -1.06321500 \\
\hline C & -3.89515400 & 7.49343600 & 1.4015900 \\
\hline
\end{tabular}




\begin{tabular}{|c|c|c|c|}
\hline C & -2.88302600 & 5.53550800 & 2.61973800 \\
\hline C & -4.31656600 & 7.45640500 & -0.97030700 \\
\hline C & -3.78311800 & 5.46338600 & -2.41156400 \\
\hline C & -4.36244700 & 8.12374100 & 0.25031500 \\
\hline $\mathrm{H}$ & -3.93498400 & 8.01034300 & 2.35735200 \\
\hline $\mathrm{H}$ & -3.37479700 & 4.56955800 & 2.78392900 \\
\hline $\mathrm{H}$ & -1.80514600 & 5.34048000 & 2.58603400 \\
\hline $\mathrm{H}$ & -3.08357300 & 6.16860500 & 3.48937300 \\
\hline $\mathrm{H}$ & -4.67434100 & 7.94914400 & -1.87114900 \\
\hline $\mathrm{H}$ & -3.95162200 & 6.18712300 & -3.21517200 \\
\hline $\mathrm{H}$ & -2.82497400 & 4.96655400 & -2.59668100 \\
\hline $\mathrm{H}$ & -4.55802800 & 4.69205800 & -2.49014400 \\
\hline $\mathrm{H}$ & -4.75982600 & 9.13394700 & 0.30490100 \\
\hline C & 6.54542400 & 0.10762000 & -0.02678900 \\
\hline C & 7.26253800 & -0.27774000 & -1.18388400 \\
\hline C & 7.21825100 & 0.29994700 & 1.20217800 \\
\hline C & 8.64699500 & -0.45131700 & -1.09525500 \\
\hline C & 6.56563900 & -0.47182900 & -2.51180300 \\
\hline C & 8.60379700 & 0.10006900 & 1.24970100 \\
\hline C & 6.48431200 & 0.69655900 & 2.46637700 \\
\hline C & 9.31691600 & -0.26796400 & 0.11256400 \\
\hline $\mathrm{H}$ & 9.20229400 & -0.73408300 & -1.98626600 \\
\hline $\mathrm{H}$ & 5.86589300 & -1.31473200 & -2.48251000 \\
\hline $\mathrm{H}$ & 5.98375000 & 0.41341300 & -2.79422700 \\
\hline $\mathrm{H}$ & 7.29261100 & -0.66523900 & -3.30638300 \\
\hline $\mathrm{H}$ & 9.12374600 & 0.23508600 & 2.19506700 \\
\hline $\mathrm{H}$ & 7.11630100 & 0.52773000 & 3.34392500 \\
\hline $\mathrm{H}$ & 6.20407900 & 1.75627500 & 2.45742900 \\
\hline $\mathrm{H}$ & 5.55827000 & 0.12777100 & 2.59944700 \\
\hline $\mathrm{H}$ & 10.39252700 & -0.41448400 & 0.16713000 \\
\hline C & -3.14871000 & -5.61276600 & 0.18374200 \\
\hline C & -3.28749900 & -6.00865100 & 1.53311900 \\
\hline
\end{tabular}




$\begin{array}{llll}\text { C } & -3.84096300 & -6.29859700 & -0.84021900 \\ \text { C } & -4.12013900 & -7.09456500 & 1.83350000 \\ \text { C } & -2.57328500 & -5.28579600 & 2.65575800 \\ \text { C } & -4.65260000 & -7.38484300 & -0.49827100 \\ \text { C } & -3.69630800 & -5.89108900 & -2.28919000 \\ \text { C } & -4.79532900 & -7.78257200 & 0.82934300 \\ \text { H } & -4.23753500 & -7.39756100 & 2.87119200 \\ \text { H } & -1.50468500 & -5.52930100 & 2.67950600 \\ \text { H } & -2.64585900 & -4.19793900 & 2.55348900 \\ \text { H } & -2.99993600 & -5.56512300 & 3.62422100 \\ \text { H } & -5.17785200 & -7.92078600 & -1.28514200 \\ \text { H } & -4.27175500 & -6.55642500 & -2.93989800 \\ \text { H } & -4.04805200 & -4.86661600 & -2.45746000 \\ \text { H } & -2.64926300 & -5.92355100 & -2.61324900 \\ \text { H } & -5.43500600 & -8.62463400 & 1.08058900\end{array}$

3T•3H $\mathrm{H}^{+}$: B3LYP/6-31G(d) level (found 0 imaginary frequencies)

Optimization energy Eopt $=-2364.3492$ Hartree

$$
31
$$

\begin{tabular}{|c|c|c|c|}
\hline Atom & $\mathbf{X}$ & $\mathbf{Y}$ & $\mathbf{Z}$ \\
\hline C & -0.24060400 & -2.85562800 & 1.04896400 \\
\hline C & 1.14145900 & -2.96092300 & 1.06783500 \\
\hline C & 1.80982300 & -3.88257600 & 0.23039300 \\
\hline C & 1.02883400 & -4.75706300 & -0.55340200 \\
\hline C & -0.35943900 & -4.64411200 & -0.58106100 \\
\hline C & -1.01253400 & -3.66035500 & 0.18565600 \\
\hline $\mathrm{H}$ & -0.72697300 & -2.17144000 & 1.73906900 \\
\hline $\mathrm{H}$ & 1.71119300 & -2.35841000 & 1.76838100 \\
\hline $\mathrm{H}$ & 1.51916700 & -5.49342800 & -1.18220100 \\
\hline $\mathrm{H}$ & -0.93225000 & -5.28781800 & -1.24140500 \\
\hline C & -3.85578800 & 2.66517600 & 0.43167700 \\
\hline$c$ & -2.66495100 & 2.69444200 & -0.31655400 \\
\hline
\end{tabular}




\begin{tabular}{|c|c|c|c|}
\hline C & -2.34035200 & 1.58208400 & -1.11866400 \\
\hline C & -3.13618800 & 0.44743000 & -1.11045000 \\
\hline C & -4.28070100 & 0.37153100 & -0.28541600 \\
\hline C & -4.64996500 & 1.51914100 & 0.44722200 \\
\hline $\mathrm{H}$ & -4.13606100 & 3.51811100 & 1.04227300 \\
\hline $\mathrm{H}$ & -1.48442600 & 1.62813300 & -1.78577600 \\
\hline $\mathrm{H}$ & -2.89751400 & -0.37389400 & -1.77857600 \\
\hline $\mathrm{H}$ & -5.54056400 & 1.49321600 & 1.06710800 \\
\hline C & 2.51214200 & 3.53464800 & -0.27104400 \\
\hline C & 3.57439400 & 3.26265700 & 0.61620400 \\
\hline C & 4.16690100 & 2.00273100 & 0.65848900 \\
\hline C & 3.71754200 & 0.97460400 & -0.18957100 \\
\hline C & 2.73945800 & 1.27831100 & -1.15968600 \\
\hline C & 2.15258200 & 2.53497000 & -1.20334800 \\
\hline $\mathrm{H}$ & 3.89365600 & 4.02777800 & 1.31632400 \\
\hline $\mathrm{H}$ & 4.93787700 & 1.80162200 & 1.39585500 \\
\hline $\mathrm{H}$ & 2.46648500 & 0.53777300 & -1.90685100 \\
\hline $\mathrm{H}$ & 1.43561100 & 2.76119400 & -1.98691400 \\
\hline C & -4.29773200 & -2.09016400 & -0.07865600 \\
\hline C & -2.44470900 & -3.39725500 & 0.03337900 \\
\hline C & -3.54179500 & -4.23064000 & -0.31079400 \\
\hline C & -4.67657100 & -3.43817000 & -0.35708200 \\
\hline C & 4.18012700 & -0.40477900 & -0.01472400 \\
\hline C & 5.43381800 & -0.94568500 & 0.37165400 \\
\hline C & 5.30337400 & -2.32372600 & 0.41647900 \\
\hline C & 3.95417500 & -2.66025900 & 0.09628400 \\
\hline C & -0.62070400 & 5.79212800 & -0.25382900 \\
\hline C & 0.35140100 & 4.74637200 & -0.19197400 \\
\hline C & -1.71715300 & 3.81280400 & -0.25717100 \\
\hline C & -1.88230000 & 5.22209200 & -0.27942000 \\
\hline C & -5.03284000 & -0.89597300 & -0.14553000 \\
\hline C & 3.28594900 & -3.89397500 & 0.13216000 \\
\hline
\end{tabular}




\begin{tabular}{|c|c|c|c|}
\hline C & 1.74777100 & 4.79318700 & -0.11952400 \\
\hline $\mathrm{N}$ & -2.92228500 & -2.13867900 & 0.16757300 \\
\hline $\mathrm{N}$ & 3.32273100 & -1.44028600 & -0.17001100 \\
\hline $\mathrm{N}$ & -0.38981100 & 3.55741700 & -0.21983200 \\
\hline $\mathrm{H}$ & -3.48798000 & -5.29628600 & -0.48608000 \\
\hline $\mathrm{H}$ & -5.67900600 & -3.75294300 & -0.61244700 \\
\hline $\mathrm{H}$ & -0.38226700 & 6.84463300 & -0.32092900 \\
\hline $\mathrm{H}$ & -2.82863700 & 5.73826800 & -0.36495600 \\
\hline $\mathrm{H}$ & 6.06361200 & -3.04026600 & 0.69542300 \\
\hline $\mathrm{H}$ & 6.32757300 & -0.37087200 & 0.57186400 \\
\hline C & 4.02997300 & -5.14364700 & 0.04026600 \\
\hline C & 3.79551500 & -6.17887900 & 1.00950000 \\
\hline C & 4.99112100 & -5.33812700 & -1.00917000 \\
\hline C & 4.54914500 & -7.34545100 & 0.92814500 \\
\hline C & 2.84335100 & -6.02605900 & 2.17699100 \\
\hline C & 5.68484700 & -6.54740300 & -1.05356400 \\
\hline C & 5.22408800 & -4.36687200 & -2.15106300 \\
\hline C & 5.48303200 & -7.53644600 & -0.09289300 \\
\hline $\mathrm{H}$ & 4.41435300 & -8.11603100 & 1.68108300 \\
\hline $\mathrm{H}$ & 2.89868000 & -5.03565800 & 2.63948800 \\
\hline $\mathrm{H}$ & 1.80175900 & -6.19903000 & 1.88355600 \\
\hline $\mathrm{H}$ & 3.08329700 & -6.75975900 & 2.95030100 \\
\hline $\mathrm{H}$ & 6.38564000 & -6.72326900 & -1.86448900 \\
\hline $\mathrm{H}$ & 5.41002600 & -4.92564700 & -3.07269600 \\
\hline $\mathrm{H}$ & 4.37688600 & -3.70198500 & -2.33422700 \\
\hline $\mathrm{H}$ & 6.10701400 & -3.74056500 & -1.97998500 \\
\hline $\mathrm{H}$ & 6.04848600 & -8.46231500 & -0.14209900 \\
\hline C & -6.48651000 & -0.91077000 & -0.04800900 \\
\hline C & -7.26825500 & -0.21989400 & -1.03672100 \\
\hline C & -7.13249600 & -1.61255800 & 1.02575500 \\
\hline C & -8.65513400 & -0.28451000 & -0.94825900 \\
\hline C & -6.66276100 & 0.48978100 & -2.22957400 \\
\hline
\end{tabular}




\begin{tabular}{|c|c|c|c|}
\hline C & -8.52641900 & -1.60508100 & 1.07508700 \\
\hline C & -6.40401700 & -2.26649600 & 2.18462600 \\
\hline C & -9.28466300 & -0.96452400 & 0.09694000 \\
\hline $\mathrm{H}$ & -9.25714300 & 0.19432800 & -1.71455200 \\
\hline $\mathrm{H}$ & -6.29548100 & 1.48925100 & -1.97046200 \\
\hline $\mathrm{H}$ & -5.83073700 & -0.06572100 & -2.67336300 \\
\hline $\mathrm{H}$ & -7.41885800 & 0.61942300 & -3.00767500 \\
\hline $\mathrm{H}$ & -9.02691700 & -2.09807000 & 1.90339600 \\
\hline $\mathrm{H}$ & -6.97801500 & -2.12203700 & 3.10431900 \\
\hline $\mathrm{H}$ & -6.30314600 & -3.34873300 & 2.04426100 \\
\hline $\mathrm{H}$ & -5.40425100 & -1.85975800 & 2.35313800 \\
\hline $\mathrm{H}$ & -10.36906200 & -0.98810200 & 0.15094500 \\
\hline C & 2.43257700 & 6.04845100 & 0.18956300 \\
\hline C & 2.08246700 & 6.80845000 & 1.35166700 \\
\hline C & 3.45938100 & 6.50982400 & -0.69970300 \\
\hline C & 2.75266600 & 8.01140200 & 1.58029600 \\
\hline C & 1.11727700 & 6.33701000 & 2.42346100 \\
\hline C & 4.06258900 & 7.73562200 & -0.43412100 \\
\hline C & 3.84802900 & 5.77265500 & -1.96373100 \\
\hline C & 3.71879100 & 8.48330600 & 0.69451900 \\
\hline $\mathrm{H}$ & 2.52393400 & 8.57956000 & 2.47745500 \\
\hline $\mathrm{H}$ & 0.14333200 & 6.83303100 & 2.34216100 \\
\hline $\mathrm{H}$ & 0.94317900 & 5.25861400 & 2.40606500 \\
\hline $\mathrm{H}$ & 1.51545000 & 6.59093900 & 3.41035400 \\
\hline $\mathrm{H}$ & 4.80792800 & 8.11891800 & -1.12442300 \\
\hline $\mathrm{H}$ & 4.42065500 & 6.43200200 & -2.62026300 \\
\hline $\mathrm{H}$ & 4.47901500 & 4.90137100 & -1.75428600 \\
\hline $\mathrm{H}$ & 2.97813100 & 5.42487900 & -2.53093800 \\
\hline $\mathrm{H}$ & 4.21430300 & 9.43013100 & 0.88750200 \\
\hline $\mathrm{H}$ & -2.35843200 & -1.32857400 & 0.38041900 \\
\hline $\mathrm{H}$ & 2.34718100 & -1.34903200 & -0.41527100 \\
\hline $\mathrm{H}$ & 0.00872700 & 2.64047300 & -0.07502200 \\
\hline
\end{tabular}


4T: B3LYP/6-31G(d) level (found 0 imaginary frequencies)

Optimization energy Eopt $=-3151.1570$ Hartree

01

\begin{tabular}{|c|c|c|c|}
\hline Atom & $\mathbf{X}$ & $\mathbf{Y}$ & $\mathbf{Z}$ \\
\hline C & 0.00427600 & -6.54800500 & -0.15766500 \\
\hline C & -1.33873400 & -6.24320300 & -0.32849900 \\
\hline C & -1.81012300 & -4.92986800 & -0.15780100 \\
\hline C & -0.87402300 & -3.93399600 & 0.18741600 \\
\hline C & 0.46843200 & -4.23151600 & 0.35020400 \\
\hline C & 0.95067000 & -5.55304300 & 0.17740400 \\
\hline $\mathrm{H}$ & 0.33224100 & -7.57273500 & -0.29277700 \\
\hline $\mathrm{H}$ & -2.02647600 & -7.03851800 & -0.59787700 \\
\hline $\mathrm{H}$ & -1.23100400 & -2.92059500 & 0.33598000 \\
\hline $\mathrm{H}$ & 1.17187500 & -3.45214400 & 0.61046800 \\
\hline C & 4.92987800 & -1.81010700 & 0.15781500 \\
\hline C & 3.93400800 & -0.87399800 & -0.18738400 \\
\hline C & 4.23153400 & 0.46845500 & -0.35016800 \\
\hline C & 5.55306700 & 0.95068400 & -0.17738600 \\
\hline C & 6.54802800 & 0.00428100 & 0.15766500 \\
\hline C & 6.24321900 & -1.33872700 & 0.32849800 \\
\hline $\mathrm{H}$ & 2.92060200 & -1.23097100 & -0.33593600 \\
\hline $\mathrm{H}$ & 3.45216400 & 1.17190600 & -0.61041900 \\
\hline $\mathrm{H}$ & 7.57276200 & 0.33223800 & 0.29276200 \\
\hline $\mathrm{H}$ & 7.03853300 & -2.02647600 & 0.59786200 \\
\hline C & -4.23153400 & -0.46845500 & -0.35016800 \\
\hline C & -5.55306700 & -0.95068400 & -0.17738600 \\
\hline C & -6.54802800 & -0.00428100 & 0.15766500 \\
\hline C & -6.24321900 & 1.33872700 & 0.32849800 \\
\hline C & -4.92987800 & 1.81010700 & 0.15781500 \\
\hline C & -3.93400800 & 0.87399800 & -0.18738400 \\
\hline-1 & -3.45216400 & -1.17190600 & -0.61041900 \\
\hline
\end{tabular}




\begin{tabular}{|c|c|c|c|}
\hline $\mathrm{H}$ & -7.57276200 & -0.33223800 & 0.29276200 \\
\hline $\mathrm{H}$ & -7.03853300 & 2.02647600 & 0.59786200 \\
\hline $\mathrm{H}$ & -2.92060200 & 1.23097100 & -0.33593600 \\
\hline C & 0.87402300 & 3.93399600 & 0.18741600 \\
\hline C & -0.46843200 & 4.23151600 & 0.35020400 \\
\hline C & -0.95067000 & 5.55304300 & 0.17740400 \\
\hline C & -0.00427600 & 6.54800500 & -0.15766500 \\
\hline C & 1.33873400 & 6.24320300 & -0.32849900 \\
\hline C & 1.81012300 & 4.92986800 & -0.15780100 \\
\hline $\mathrm{H}$ & 1.23100400 & 2.92059500 & 0.33598000 \\
\hline $\mathrm{H}$ & -1.17187500 & 3.45214400 & 0.61046800 \\
\hline $\mathrm{H}$ & -0.33224100 & 7.57273500 & -0.29277700 \\
\hline $\mathrm{H}$ & 2.02647600 & 7.03851800 & -0.59787700 \\
\hline C & -3.22720900 & -4.60011200 & -0.32076000 \\
\hline C & -4.30764500 & -5.55273100 & -0.60230400 \\
\hline C & -5.44909700 & -4.82333700 & -0.64515300 \\
\hline C & -5.06071300 & -3.43112800 & -0.40067000 \\
\hline C & 4.82332800 & -5.44908100 & 0.64515800 \\
\hline C & 3.43112000 & -5.06069000 & 0.40068200 \\
\hline C & 4.60011500 & -3.22719200 & 0.32077100 \\
\hline C & 5.55273000 & -4.30763400 & 0.60230200 \\
\hline C & 5.06071300 & 3.43112800 & -0.40067000 \\
\hline C & 3.22720900 & 4.60011200 & -0.32076000 \\
\hline C & 4.30764500 & 5.55273100 & -0.60230400 \\
\hline C & 5.44909700 & 4.82333700 & -0.64515300 \\
\hline C & -4.60011500 & 3.22719200 & 0.32077100 \\
\hline C & -5.55273000 & 4.30763400 & 0.60230200 \\
\hline C & -4.82332800 & 5.44908100 & 0.64515800 \\
\hline C & -3.43112000 & 5.06069000 & 0.40068200 \\
\hline C & -5.93009600 & -2.36071100 & -0.35666600 \\
\hline C & 2.36069900 & -5.93006900 & 0.35668000 \\
\hline C & 5.93009600 & 2.36071100 & $-0.356666 c$ \\
\hline
\end{tabular}




\begin{tabular}{|c|c|c|c|}
\hline C & -2.36069900 & 5.93006900 & 0.35668000 \\
\hline V & -3.67766100 & -3.36498600 & -0.20704500 \\
\hline $\mathrm{N}$ & 3.36498600 & -3.67763700 & 0.20706100 \\
\hline $\mathrm{N}$ & 3.67766100 & 3.36498600 & -0.20704500 \\
\hline N & -3.36498600 & 3.67763700 & 0.20706100 \\
\hline - & -4.21009300 & -6.62191200 & -0.73743800 \\
\hline H & -6.45492400 & -5.17397800 & -0.83187000 \\
\hline $\mathrm{H}$ & 5.17396400 & -6.45491000 & 0.83187300 \\
\hline $\mathrm{H}$ & 6.62191200 & -4.21008900 & 0.73742900 \\
\hline $\mathrm{H}$ & 6.45492400 & 5.17397800 & -0.83187000 \\
\hline $\mathrm{H}$ & 4.21009300 & 6.62191200 & -0.73743800 \\
\hline $\mathrm{H}$ & -5.17396400 & 6.45491000 & 0.83187300 \\
\hline $\mathrm{H}$ & -6.62191200 & 4.21008900 & 0.73742900 \\
\hline C & -7.39725800 & -2.66734500 & -0.50106600 \\
\hline C & -8.14298400 & -3.08567200 & 0.62255100 \\
\hline C & -8.01655100 & -2.52743300 & -1.76251500 \\
\hline C & -9.50761400 & -3.35748700 & 0.46356900 \\
\hline C & -7.49852700 & -3.24353600 & 1.98258800 \\
\hline C & -9.38070600 & -2.81663500 & -1.88091800 \\
\hline C & -7.22888300 & -2.09151600 & -2.97758600 \\
\hline & -10.12533400 & -3.22721900 & -0.77759400 \\
\hline 11 & -10.08685000 & -3.67487500 & 1.32735300 \\
\hline $\mathrm{H}$ & -6.80378600 & -4.09143600 & 2.00314400 \\
\hline $\mathrm{H}$ & -6.92161400 & -2.35598100 & 2.26561300 \\
\hline $\mathrm{H}$ & -8.25725900 & -3.41358900 & 2.75266100 \\
\hline - & -9.85955000 & -2.71632300 & -2.85198200 \\
\hline $\mathrm{H}$ & -7.87203200 & -2.04436000 & -3.86153000 \\
\hline $\mathrm{H}$ & -6.77896500 & -1.10226400 & -2.83453600 \\
\hline $\mathrm{H}$ & -6.40734900 & -2.78456300 & -3.19381100 \\
\hline $\mathrm{H}$ & -11.18504200 & -3.44372900 & -0.88477600 \\
\hline C & 2.66733400 & -7.39723300 & 0.50106200 \\
\hline C & 2.52747000 & -8.01653100 & 1.76251300 \\
\hline
\end{tabular}




\begin{tabular}{|c|c|c|c|}
\hline C & 3.08561200 & -8.14295600 & -0.62257600 \\
\hline C & 2.81667400 & -9.38068700 & 1.88089900 \\
\hline C & 2.09160900 & -7.22886700 & 2.97760700 \\
\hline C & 3.35742800 & -9.50758700 & -0.46361100 \\
\hline C & 3.24341300 & -7.49849600 & -1.98261900 \\
\hline C & 3.22720900 & -10.12531200 & 0.77755400 \\
\hline $\mathrm{H}$ & 2.71640100 & -9.85953500 & 2.85196500 \\
\hline $\mathrm{H}$ & 2.78466800 & -6.40733500 & 3.19380400 \\
\hline $\mathrm{H}$ & 1.10235200 & -6.77894600 & 2.83460400 \\
\hline $\mathrm{H}$ & 2.04449200 & -7.87201900 & 3.86155100 \\
\hline $\mathrm{H}$ & 3.67477600 & -10.08682100 & -1.32741100 \\
\hline $\mathrm{H}$ & 3.41342600 & -8.25722700 & -2.75270200 \\
\hline $\mathrm{H}$ & 2.35584700 & -6.92157800 & -2.26560000 \\
\hline $\mathrm{H}$ & 4.09131600 & -6.80375900 & -2.00321400 \\
\hline $\mathrm{H}$ & 3.44372100 & -11.18502100 & 0.88472300 \\
\hline C & 7.39725800 & 2.66734500 & -0.50106600 \\
\hline C & 8.14298400 & 3.08567200 & 0.62255100 \\
\hline C & 8.01655100 & 2.52743300 & -1.76251500 \\
\hline C & 9.50761400 & 3.35748700 & 0.46356900 \\
\hline C & 7.49852700 & 3.24353600 & 1.98258800 \\
\hline C & 9.38070600 & 2.81663500 & -1.88091800 \\
\hline C & 7.22888300 & 2.09151600 & -2.97758600 \\
\hline C & 10.12533400 & 3.22721900 & -0.77759400 \\
\hline $\mathrm{H}$ & 10.08685000 & 3.67487500 & 1.32735300 \\
\hline $\mathrm{H}$ & 6.80378600 & 4.09143600 & 2.00314400 \\
\hline $\mathrm{H}$ & 6.92161400 & 2.35598100 & 2.26561300 \\
\hline $\mathrm{H}$ & 8.25725900 & 3.41358900 & 2.75266100 \\
\hline $\mathrm{H}$ & 9.85955000 & 2.71632300 & -2.85198200 \\
\hline $\mathrm{H}$ & 7.87203200 & 2.04436000 & -3.86153000 \\
\hline $\mathrm{H}$ & 6.77896500 & 1.10226400 & -2.83453600 \\
\hline $\mathrm{H}$ & 6.40734900 & 2.78456300 & -3.19381100 \\
\hline $\mathrm{H}$ & 11.18504200 & 3.44372900 & -0.88477600 \\
\hline
\end{tabular}




$\begin{array}{llll}\text { C } & -2.66733400 & 7.39723300 & 0.50106200 \\ \text { C } & -3.08561200 & 8.14295600 & -0.62257600 \\ \text { C } & -2.52747000 & 8.01653100 & 1.76251300 \\ \text { C } & -3.35742800 & 9.50758700 & -0.46361100 \\ \text { C } & -3.24341300 & 7.49849600 & -1.98261900 \\ \text { C } & -2.81667400 & 9.38068700 & 1.88089900 \\ \text { C } & -2.09160900 & 7.22886700 & 2.97760700 \\ \text { C } & -3.22720900 & 10.12531200 & 0.77755400 \\ \text { H } & -3.67477600 & 10.08682100 & -1.32741100 \\ \text { H } & -2.35584700 & 6.92157800 & -2.26560000 \\ \text { H } & -4.09131600 & 6.80375900 & -2.00321400 \\ \text { H } & -3.41342600 & 8.25722700 & -2.75270200 \\ \text { H } & -2.71640100 & 9.85953500 & 2.85196500 \\ \text { H } & -2.04449200 & 7.87201900 & 3.86155100 \\ \text { H } & -2.78466800 & 6.40733500 & 3.19380400 \\ \text { H } & -1.10235200 & 6.77894600 & 2.83460400 \\ \text { H } & -3.44372100 & 11.18502100 & 0.88472300\end{array}$

4T•4H+: B3LYP/6-31G(d) level (found 0 imaginary frequencies)

Optimization energy Eopt $=-3152.4338$ Hartree

41

\begin{tabular}{|c|c|c|c|}
\hline Atom & $\mathbf{X}$ & $\mathbf{Y}$ & $\mathbf{Z}$ \\
\hline C & -0.92129600 & 6.23309300 & 0.83615300 \\
\hline C & -2.15684500 & 5.64770100 & 1.06975300 \\
\hline C & -2.54300300 & 4.47317200 & 0.39070600 \\
\hline C & -1.62978500 & 3.89827500 & -0.51835200 \\
\hline C & -0.38516000 & 4.47321400 & -0.73958000 \\
\hline C & -0.00465400 & 5.65987800 & -0.07168400 \\
\hline $\mathrm{H}$ & -0.64962300 & 7.13910400 & 1.36718900 \\
\hline $\mathrm{H}$ & -2.82473000 & 6.10466100 & 1.79170800 \\
\hline $\mathrm{H}$ & -1.90974300 & 3.03024200 & -1.10902300 \\
\hline $\mathrm{H}$ & 0.26380700 & 4.04969400 & -1.50028700 \\
\hline
\end{tabular}




\begin{tabular}{|c|c|c|c|}
\hline C & 4.47318800 & 2.54303200 & -0.39066300 \\
\hline C & 3.89825900 & 1.62979200 & 0.51835500 \\
\hline C & 4.47318800 & 0.38516200 & 0.73957400 \\
\hline C & 5.65987500 & 0.00466700 & 0.07171100 \\
\hline C & 6.23312300 & 0.92133200 & -0.83608300 \\
\hline C & 5.64774100 & 2.15688800 & -1.06967400 \\
\hline $\mathrm{H}$ & 3.03020400 & 1.90973700 & 1.10900100 \\
\hline $\mathrm{H}$ & 4.04963900 & -0.26382000 & 1.50025200 \\
\hline $\mathrm{H}$ & 7.13915000 & 0.64967000 & -1.36709700 \\
\hline $\mathrm{H}$ & 6.10473000 & 2.82478900 & -1.79159600 \\
\hline C & -4.47318800 & -0.38516200 & 0.73957400 \\
\hline C & -5.65987500 & -0.00466700 & 0.07171100 \\
\hline C & -6.23312300 & -0.92133200 & -0.83608300 \\
\hline C & -5.64774100 & -2.15688800 & -1.06967400 \\
\hline C & -4.47318800 & -2.54303200 & -0.39066300 \\
\hline C & -3.89825900 & -1.62979200 & 0.51835500 \\
\hline $\mathrm{H}$ & -4.04963900 & 0.26382000 & 1.50025200 \\
\hline $\mathrm{H}$ & -7.13915000 & -0.64967000 & -1.36709700 \\
\hline $\mathrm{H}$ & -6.10473000 & -2.82478900 & -1.79159600 \\
\hline $\mathrm{H}$ & -3.03020400 & -1.90973700 & 1.10900100 \\
\hline C & 1.62978500 & -3.89827500 & -0.51835200 \\
\hline C & 0.38516000 & -4.47321400 & -0.73958000 \\
\hline C & 0.00465400 & -5.65987800 & -0.07168400 \\
\hline C & 0.92129600 & -6.23309300 & 0.83615300 \\
\hline C & 2.15684500 & -5.64770100 & 1.06975300 \\
\hline C & 2.54300300 & -4.47317200 & 0.39070600 \\
\hline $\mathrm{H}$ & 1.90974300 & -3.03024200 & -1.10902300 \\
\hline $\mathrm{H}$ & -0.26380700 & -4.04969400 & -1.50028700 \\
\hline $\mathrm{H}$ & 0.64962300 & -7.13910400 & 1.36718900 \\
\hline $\mathrm{H}$ & 2.82473000 & -6.10466100 & 1.79170800 \\
\hline C & -3.87458600 & 3.91809000 & 0.61754900 \\
\hline C & -5.00421900 & 4.55092000 & 1.20210900 \\
\hline
\end{tabular}




\begin{tabular}{|c|c|c|c|}
\hline C & -6.06347800 & 3.66889900 & 1.14651200 \\
\hline C & -5.60928700 & 2.45750400 & 0.54754000 \\
\hline C & 3.66892700 & 6.06351400 & -1.14644600 \\
\hline C & 2.45751700 & 5.60931200 & -0.54751100 \\
\hline C & 3.91811100 & 3.87461700 & -0.61749900 \\
\hline C & 4.55095000 & 5.00425600 & -1.20203700 \\
\hline C & 5.60928700 & -2.45750400 & 0.54754000 \\
\hline C & 3.87458600 & -3.91809000 & 0.61754900 \\
\hline C & 5.00421900 & -4.55092000 & 1.20210900 \\
\hline C & 6.06347800 & -3.66889900 & 1.14651200 \\
\hline C & -3.91811100 & -3.87461700 & -0.61749900 \\
\hline C & -4.55095000 & -5.00425600 & -1.20203700 \\
\hline C & -3.66892700 & -6.06351400 & -1.14644600 \\
\hline C & -2.45751700 & -5.60931200 & -0.54751100 \\
\hline C & -6.33336100 & 1.28187000 & 0.31648800 \\
\hline C & 1.28187600 & 6.33337900 & -0.31647000 \\
\hline C & 6.33336100 & -1.28187000 & 0.31648800 \\
\hline C & -1.28187600 & -6.33337900 & -0.31647000 \\
\hline$N$ & -4.25351300 & 2.66327300 & 0.25879000 \\
\hline $\mathrm{N}$ & 2.66329000 & 4.25354200 & -0.25875100 \\
\hline$N$ & 4.25351300 & -2.66327300 & 0.25879000 \\
\hline $\mathrm{N}$ & -2.66329000 & -4.25354200 & -0.25875100 \\
\hline $\mathrm{H}$ & -5.02633800 & 5.55470900 & 1.60122200 \\
\hline $\mathrm{H}$ & -7.06419300 & 3.82576600 & 1.52388300 \\
\hline $\mathrm{H}$ & 3.82580200 & 7.06423200 & -1.52380400 \\
\hline $\mathrm{H}$ & 5.55474700 & 5.02638200 & -1.60112900 \\
\hline $\mathrm{H}$ & 7.06419300 & -3.82576600 & 1.52388300 \\
\hline $\mathrm{H}$ & 5.02633800 & -5.55470900 & 1.60122200 \\
\hline $\mathrm{H}$ & -3.82580200 & -7.06423200 & -1.52380400 \\
\hline $\mathrm{H}$ & -5.55474700 & -5.02638200 & -1.60112900 \\
\hline C & -7.79776700 & 1.34874400 & 0.29917900 \\
\hline C & -8.46572400 & 2.26485200 & -0.57816700 \\
\hline
\end{tabular}




\begin{tabular}{|c|c|c|c|}
\hline C & -8.55329500 & 0.49264000 & 1.16795000 \\
\hline C & -9.86097900 & 2.29715700 & -0.56178400 \\
\hline C & -7.76171500 & 3.12688900 & -1.60913700 \\
\hline C & -9.94061300 & 0.60338600 & 1.16354000 \\
\hline C & -7.91245500 & -0.45275200 & 2.16271600 \\
\hline C & -10.59514200 & 1.49027900 & 0.30500700 \\
\hline $\mathrm{H}$ & -10.38117000 & 2.95748900 & -1.24966400 \\
\hline $\mathrm{H}$ & -7.63311200 & 4.15720200 & -1.25764400 \\
\hline $\mathrm{H}$ & -6.77694600 & 2.74675900 & -1.89165900 \\
\hline $\mathrm{H}$ & -8.36725800 & 3.17940600 & -2.51844000 \\
\hline $\mathrm{H}$ & -10.52237500 & -0.00379500 & 1.85060000 \\
\hline $\mathrm{H}$ & -8.62646200 & -0.70425300 & 2.95075900 \\
\hline $\mathrm{H}$ & -7.60702100 & -1.39569000 & 1.69439300 \\
\hline $\mathrm{H}$ & -7.03100700 & -0.02092800 & 2.64715600 \\
\hline $\mathrm{H}$ & -11.67960500 & 1.54693500 & 0.30999900 \\
\hline C & 1.34873700 & 7.79778200 & -0.29918100 \\
\hline C & 0.49261900 & 8.55328200 & -1.16797100 \\
\hline C & 2.26487400 & 8.46577200 & 0.57810600 \\
\hline C & 0.60340000 & 9.94059400 & -1.16364500 \\
\hline C & -0.45279200 & 7.91239500 & -2.16268700 \\
\hline C & 2.29722100 & 9.86102900 & 0.56163300 \\
\hline C & 3.12689800 & 7.76182900 & 1.60913200 \\
\hline C & 1.49034000 & 10.59515600 & -0.30517700 \\
\hline $\mathrm{H}$ & -0.00378800 & 10.52233200 & -1.85071800 \\
\hline $\mathrm{H}$ & -0.02082200 & 7.03113500 & -2.64734800 \\
\hline $\mathrm{H}$ & -1.39558000 & 7.60664200 & -1.69427600 \\
\hline $\mathrm{H}$ & -0.70458600 & 8.62647500 & -2.95056900 \\
\hline $\mathrm{H}$ & 2.95758500 & 10.38124200 & 1.24946700 \\
\hline $\mathrm{H}$ & 3.17906500 & 8.36724100 & 2.51854500 \\
\hline $\mathrm{H}$ & 2.74701000 & 6.77690400 & 1.89141900 \\
\hline $\mathrm{H}$ & 4.15731600 & 7.63359800 & 1.25781000 \\
\hline $\mathrm{H}$ & 1.54702000 & 11.67961700 & -0.31023900 \\
\hline
\end{tabular}




\begin{tabular}{|c|c|c|c|}
\hline C & 7.79776700 & -1.34874400 & 0.29917900 \\
\hline C & 8.46572400 & -2.26485200 & -0.57816700 \\
\hline C & 8.55329500 & -0.49264000 & 1.16795000 \\
\hline C & 9.86097900 & -2.29715700 & -0.56178400 \\
\hline C & 7.76171500 & -3.12688900 & -1.60913700 \\
\hline C & 9.94061300 & -0.60338600 & 1.16354000 \\
\hline C & 7.91245500 & 0.45275200 & 2.16271600 \\
\hline C & 10.59514200 & -1.49027900 & 0.30500700 \\
\hline $\mathrm{H}$ & 10.38117000 & -2.95748900 & -1.24966400 \\
\hline $\mathrm{H}$ & 7.63311200 & -4.15720200 & -1.25764400 \\
\hline $\mathrm{H}$ & 6.77694600 & -2.74675900 & -1.89165900 \\
\hline $\mathrm{H}$ & 8.36725800 & -3.17940600 & -2.51844000 \\
\hline $\mathrm{H}$ & 10.52237500 & 0.00379500 & 1.85060000 \\
\hline $\mathrm{H}$ & 8.62646200 & 0.70425300 & 2.95075900 \\
\hline $\mathrm{H}$ & 7.60702100 & 1.39569000 & 1.69439300 \\
\hline $\mathrm{H}$ & 7.03100700 & 0.02092800 & 2.64715600 \\
\hline $\mathrm{H}$ & 11.67960500 & -1.54693500 & 0.30999900 \\
\hline C & -1.34873700 & -7.79778200 & -0.29918100 \\
\hline C & -2.26487400 & -8.46577200 & 0.57810600 \\
\hline C & -0.49261900 & -8.55328200 & -1.16797100 \\
\hline C & -2.29722100 & -9.86102900 & 0.56163300 \\
\hline C & -3.12689800 & -7.76182900 & 1.60913200 \\
\hline C & -0.60340000 & -9.94059400 & -1.16364500 \\
\hline C & 0.45279200 & -7.91239500 & -2.16268700 \\
\hline C & -1.49034000 & -10.59515600 & -0.30517700 \\
\hline $\mathrm{H}$ & -2.95758500 & -10.38124200 & 1.24946700 \\
\hline $\mathrm{H}$ & -2.74701000 & -6.77690400 & 1.89141900 \\
\hline $\mathrm{H}$ & -4.15731600 & -7.63359800 & 1.25781000 \\
\hline $\mathrm{H}$ & -3.17906500 & -8.36724100 & 2.51854500 \\
\hline $\mathrm{H}$ & 0.00378800 & -10.52233200 & -1.85071800 \\
\hline $\mathrm{H}$ & 0.70458600 & -8.62647500 & -2.95056900 \\
\hline $\mathrm{H}$ & 0.02082200 & -7.03113500 & -2.64734800 \\
\hline
\end{tabular}




$\begin{array}{lrrr}\mathrm{H} & 1.39558000 & -7.60664200 & -1.69427600 \\ \mathrm{H} & -1.54702000 & -11.67961700 & -0.31023900 \\ \mathrm{H} & 1.99228200 & 3.67475100 & 0.22610500 \\ \mathrm{H} & -3.67472900 & 1.99227800 & -0.22609100 \\ \mathrm{H} & 3.67472900 & -1.99227800 & -0.22609100 \\ \mathrm{H} & -1.99228200 & -3.67475100 & 0.22610500\end{array}$

\section{Supporting References}

[S1] Gaussian 16, Revision C.01, Frisch MJ, Trucks GW, Schlegel HB, Scuseria GE, Robb MA, Cheeseman JR, Scalmani G, Barone V, Petersson GA, NakatsujiH, Li X, Caricato M, Marenich AV, Bloino J, Janesko BG, Gomperts R, Mennucci B, Hratchian HP, Ortiz JV, Izmaylov AF, Sonnenberg JL, Williams-Young D, Ding F, Lipparini F, Egidi F, Goings J, Peng B, Petrone A, Henderson T, Ranasinghe D, Zakrzewski VG, Gao J, Rega N, Zheng G, Liang W, Hada M, Ehara M, Toyota K, Fukuda R, Hasegawa J, Ishida M, Nakajima T, Honda Y, Kitao O, Nakai H, Vreven T, Throssell K, Montgomery JA Jr, Peralta JE, Ogliaro F, Bearpark M, Heyd JJ, Brothers EN, Kudin KN, Staroverov VN, Keith T, Kobayashi R, Normand J, Raghavachari K, Rendell A, Burant JC, Iyengar SS, Tomasi J, Cossi M, Millam J M, Klene M, Adamo C, Cammi R, Ochterski JW, Martin RL, Morokuma K, Farkas O, Foresman JB, Fox DJ. Gaussian, Inc., Wallingford CT, 2016.

[S2] (a) A. D. Becke, J. Chem. Phys., 1993, 98, 1372; (b) C. Lee, W. Yang and R. G. Parr, Phys. Rev. B, 1988, 37, 785.

[S3] (a) R. Bauernschmitt, R. Ahlrichs, Chem. Phys. Lett. 1996, 256, 454-464; (b) R. E. Stratmann, G. E. Scuseria, M. J. Frisch, J. Chem. Phys. 1998, 109, 8218-8224; Cc) M. E. Casida, C. Jamorski, K. C. Casida, D. R. Salahub, J. Chem. Phys. 1998, 108, 4439-4449.

[S4] CrystalClear-SM Expert 2.1 b46: Reflection collection and data reduction package (Rigaku, 2016).

[S5] CrysAlisPro 1.171.41.93a: Data reduction package (Rigaku OD, 2020).

[S6] SHELXT 2018/2: Integrated space-group and crystal-structure determination; Sheldrick, G. M. Acta Cryst. 2015, A71, 3-8.

[S7] Olex2 1.5: A complete structure solution, refinement and analysis program; Dolomanov, O.V.; Bourhis, L.J.; Gildea, R.J.; Howard, J.A.K.; Puschmann, H. J. Appl. Cryst., 2009, 42, 339-341.

[S8] SHELXL-2018/3: Crystal structure refinement with SHELXL; Sheldrick, G. M. Acta Cryst. 2015, C71, 3-8.

[S9] PLATON: Structure validation in chemical crystallography; Spek, A. L. Acta Cryst. 2009, D65, 148-155.

[S10] (a) Martina S, Enkelmann V, Wegner G and Schlüter A.-D. Synthesis, 1991; 613. (b) Zhong Z, Lin G.-Q, Sun Z.-H and Wang B. Acta Cryst. 2009; E65: 687. 